BDX-613-1583 (Rev.)

Distribution Category UC-38

\title{
DYNAMIC RESPONSE OF RIGID POLYURETHANE FOAM
}

Published September 1976

Project Leader:

R. A. Daniel

Department. 861

Project Team:

J. R. Fender

R. D. Jump

L. F. Thorne

PDO 6984191

Topical Report

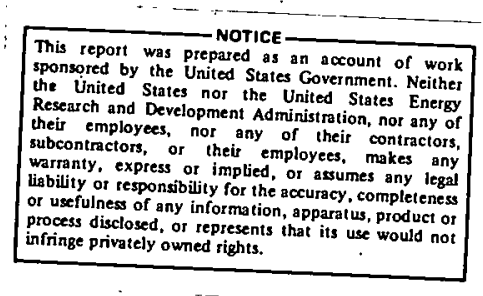

Technical Communications

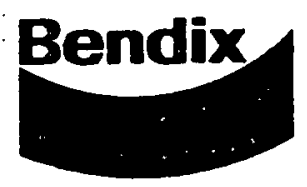

\section{Kansas City Division}




\section{DISCLAIMER}

This report was prepared as an account of work sponsored by an agency of the United States Government. Neither the United States Government nor any agency Thereof, nor any of their employees, makes any warranty, express or implied, or assumes any legal liability or responsibility for the accuracy, completeness, or usefulness of any information, apparatus, product, or process disclosed, or represents that its use would not infringe privately owned rights. Reference herein to any specific commercial product, process, or service by trade name, trademark, manufacturer, or otherwise does not necessarily constitute or imply its endorsement, recommendation, or favoring by the United States Government or any agency thereof. The views and opinions of authors expressed herein do not necessarily state or reflect those of the United States Government or any agency thereof. 


\section{DISCLAIMER}

Portions of this document may be illegible in electronic image products. Images are produced from the best available original document. 


\section{DYNAMIC RESPONSE OF RIGID POLYURETHANE FOAM}

\section{BDX-613-1583 (Rev.), UNCLASSIFIED Topical Report, Published September 1976}

Prepared by R. A. Daniel, D/861, under PDO 6984191

The dynamic characteristics of six rigid polyurethane foams were studied at impact velocities from 15.24 to $60.96 \mathrm{~m} / \mathrm{s}$ ( 50 to $200 \mathrm{ft} / \mathrm{sec})$. A test technique developed for crushing confined samples is described. The dynamic properties of materials tested are reported by both graphical and tabular methods.

This report was prepared as an account of work sponsored by the United States Government. Neither the United States nor the United States Energy Research and Development Administration, nor any of their employees, nor any of their contractors, subcontractors, or their employees, makes any warranty, express or implied, or assumes any legal liability or responsibility for the accuracy, completeness or usefulness of any information, apparatus, product or process disclosed, or represents that its use would not infringe privately owned rights.
THE BENDIX CORPORATION

KANSAS CITY DIVISION

P.O. BOX 1159

KANSAS CITY, MISSOURI 64141

\footnotetext{
A prime contractor for the United States Energy Research and Development Administration Contract Number E(29-1)-613 USERDA
}

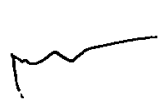


CONTENTS

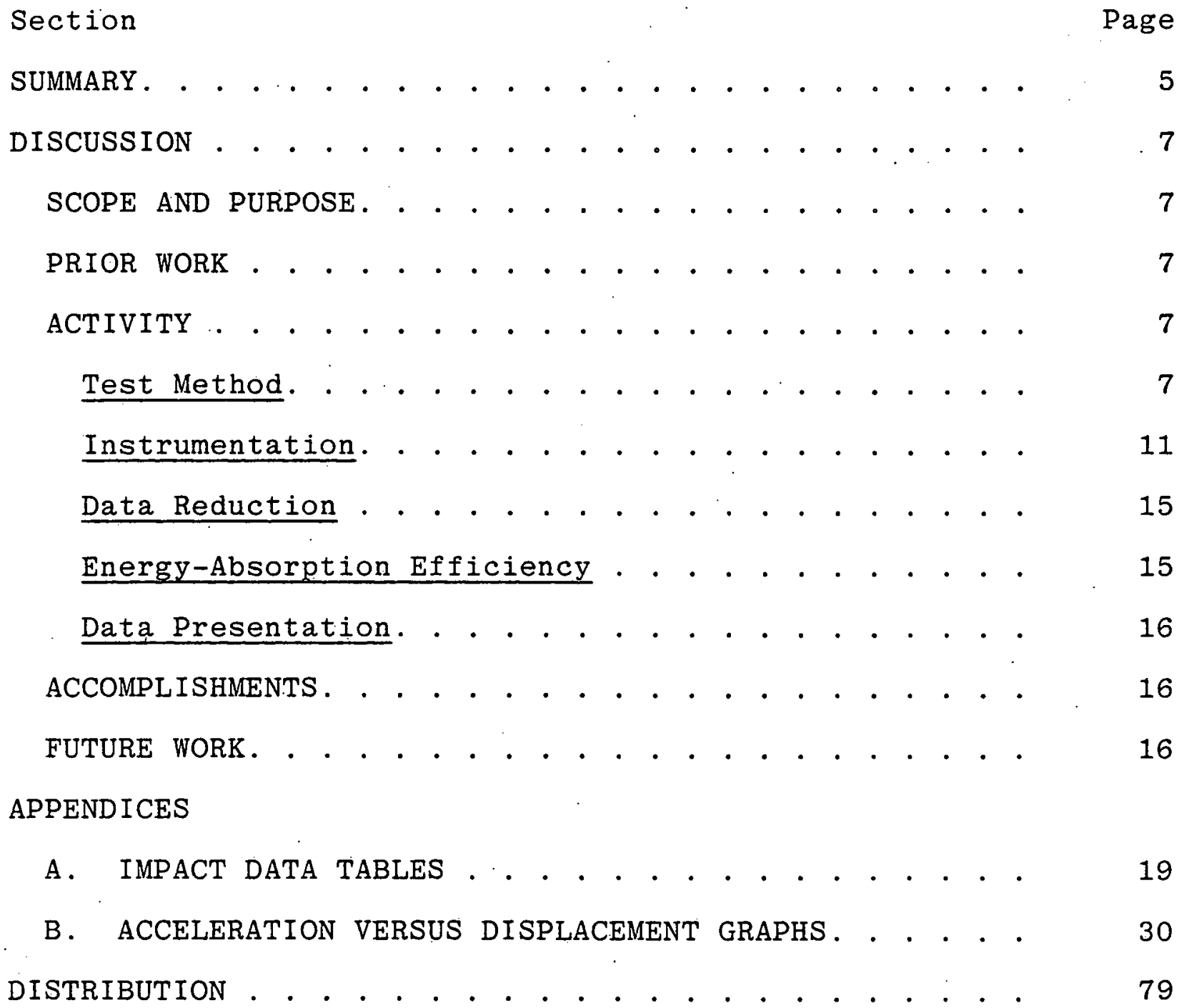




\section{ILLUSTRATIONS}

Figure

Page

1

Basic Concept for Dynamic Testing of Foam. .

Test Weight Guide Tube Attached to Carriage (P-92544). . . . . . . . . . . . . .

Test Weight with Sample Prepared for Test $(\mathrm{p}-92543)$. . . . . . . . . . . . . .

Velocity Calculation From Typical Output of Magnetic Sensors (Tracing) . . . . . . . .

Block Diagram of Instrumentation System. . .

\section{TABLES}

Number

Page

1

Energy Related to Test Weights and Impact

Velocities.... . . . . . . . . . . .

Effect of Input-Parameter Errors Upon Accuracy of Output Data. . . . . . . . . 18

A-1 Impact Data for CPR 1024 . . . . . . . . . 20

A-2 Impact Data for CPR 1040 . . . . . . . . . . 21

A-3 Impact Data for BC 1200 Series . . . . . . . . 22

A-4 Impact Data for BKC 4003 . . . . . . . . . . 24

A-5 Impact Data for BKC 6003 . . . . . . . . . . 26

A-6 Impact Data for BKC 44302. . . . . . . . . . . 28 


\section{SUMMARY}

Development of components for future applications is dependent on complete and precise data on the response of materials to various environments. The energy absorption of polyurethane foam material had previously been investigated at velocities below $6 \mathrm{~m} / \mathrm{s}$ (20 ft/sec) and above several thousand meters/second (feet/second). However, very little work had been performed in the velocity range applicable to future designs. The purpose of this project was to develop the techniques to evaluate polyurethane foam response to impact velocities in the range of 15.24 to $91.44 \mathrm{~m} / \mathrm{s}$ ( 50 to $300 \mathrm{ft} / \mathrm{sec}$ ) and to report these data in a format usable for present and future design activities.

During the initial phase of this work, a technique for testing dynamic response from 15.24 to $60.96 \mathrm{~m} / \mathrm{s}$ (50 to $200 \mathrm{ft} / \mathrm{sec}$ ) was established, and two techniques were proposed for reporting these data. This work was documented in BDX-613-1059 (Rev.), August 1975 .

The second phase of this project involved testing rigid polyurethane foam samples $63.5 \mathrm{~mm}$ ( 2.5 inches) in diameter and $101.6 \mathrm{~mm}$ ( 4 inches) long. The samples were placed inside a steel tube to prevent lateral expansion and were crushed in the longitudinal direction of impact velocities of $15.24,30.48$, 45.72 , and $60.96 \mathrm{~m} / \mathrm{s}(50,100,150$, and $200 \mathrm{ft} / \mathrm{sec})$. The materials selected for testing were CPR 1024 and 1040 (CPR Division of the UpJohn Company), BC 1200 series. (Expanded Rubber Company), BKC Thermalthane 4003, BKC Rigifoam 6003, and BKC 44302 (Bendix Corporation Kansas City Division). Samples were prepared from billets with nominal densities of 160,320 , and $480 \mathrm{~kg} / \mathrm{m}^{3}$ $\left(10,20\right.$, and $\left.301 \mathrm{~b} / \mathrm{ft}^{3}\right)$.

The impact tests were performed by using test weights of 5.4 , 10.8 , and $21.6 \mathrm{~kg}(2.45,4.91$, and $9.82 \mathrm{lb})$, corresponding to static loads of $3.4,6.9$, and $13.8 \mathrm{kPa}(0.5,1.0$, and $2.0 \mathrm{psi})$. The output from an accelerometer attached to the test weight was recorded on magnetic tape. All values used to describe the dynamic behavior of the test samples were calculated from measurement of the impact velocity and the record of acceleration experienced by the test weight as it crushed the samples.

For each combination of foam type, sample density, and static load, graphs were plotted showing force versus sample deflection at $15.24,30.48,45.72$, and $60.96 \mathrm{~m} / \mathrm{s}(50,100,150$, and $200 \mathrm{ft} / \mathrm{sec}$. In addition, the force-deflection curve from a standard compression test is included for comparison with the dynamic data. 
Test results are presented in six tables which show values for maximum test weight acceleration, maximum and final sample deflection, rebound velocity, and energy absorption efficiency.

No future work is currently planned for this project. Additional studies, if desired, will be supported by other projects. 


\section{DISCUSSION}

\section{SCOPE AND PURPOSE}

Advanced product development is dependent on complete and precise data on all materials and environments. In support of this development, work was initiated to establish techniques for evaluation of foam response to impact velocities in the range of 15.24 to $91.44 \mathrm{~m} / \mathrm{s}$ ( 50 to $300 \mathrm{ft} / \mathrm{sec}$ ), and for reporting these data in a format usable to the designer specifying polyurethane foams for structural and energy absorbing media.

\section{PRIOR WORK}

Previous investigations revealed that available literature on the dynamic response of polyurethane foam discussed response at impact velocities below $6 \mathrm{~m} / \mathrm{s}$ ( $20 \mathrm{ft} / \mathrm{sec}$ ) and at several thousand ft/sec. The initial phase of this endeavor, which established the required techniques to allow testing in the range of 15.24 to $60.96 \mathrm{~m} / \mathrm{s}$ ( 50 to $2.00 \mathrm{ft} / \mathrm{sec}$ ) was reported in BDX-613-1059 (Rev.), Technique for Impact Testing of Confined Rigid Foam, August 1975.

\section{ACTIVITY}

\section{Test Method}

The basic concept used for gathering dynamic test data from foam samples is illustrated in Figure 1 . All values for the terms used to describe the dynamic behavior of a test sample can be calculated from a measurement of the impact velocity and a record of the deceleration experienced by the test weight as it crushes the sample. Samples chosen for the study were $63.5 \mathrm{~mm}$ (2.5 inches) in diameter and $101.6 \mathrm{~mm}$ ( 4 inches) in length.

The materials selected for evaluation included those most frequently used as support materials: CPR 1024 and 1040 (CPR Division of the UpJohn Company), BC 1200 series (Expanded Rubber Company), BKC Thermalthane 4003, BKC Rigifoam 6003, and BKC 44302 (Bendix Corporation, Kansas City Division). Samples were prepared from billets with nominal densities of 160,320 , and $480 \mathrm{~kg} / \mathrm{m}^{3}(10$, 20 , and $301 \mathrm{~b} / \mathrm{ft}^{3}$ ).

For each test condition, consisting of an impact load, foam type, and sample density, the impact velocity was increased in increments of $15.24 \mathrm{~m} / \mathrm{s}$ (50 ft/sec) until a sample was crushed beyond critical deflection. A new sample was used for each impact. The point of critical deflection can be determined from the accelerationtime trace by a rapid rise in the acceleration level. The energy 


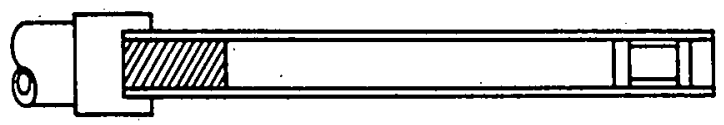

A. SAMPLE READY FOR TEST

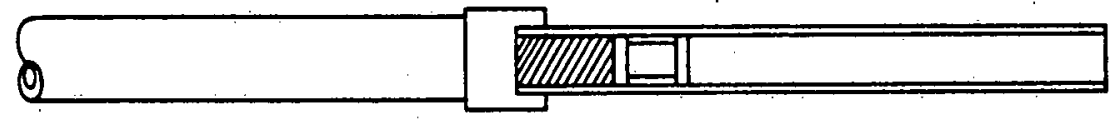

B. SAMPLE IMPACTS TEST WEIGHT

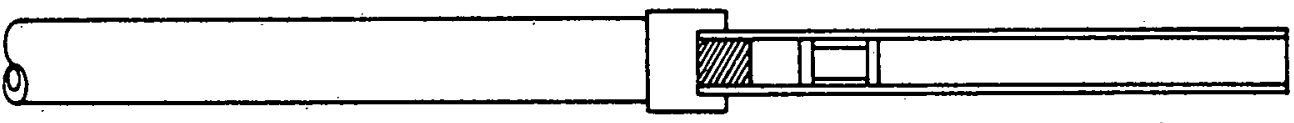

C. SAMPLE CRUSHED; TEST WEIGHT LEAVES TUBE

Figure 1. Basic Concept for Dynamic Testing of Foam

absorbing efficiency of the foam diminishes rapidly after critical deflection has been exceeded.

Impact testing was conducted through the use of a pneumatic mechanical shock actuator. A guide tube 1.5 meters ( 5 ft) long with a 76.2-mm (3-in.) inside diameter, was attached to the actuator carriage which travels on a horizontal track system. The guide tube, carriage, and actuator are shown in Figure 2 . Three test weights were fabricated, weighing $5.4,10.8$, and $21.6 \mathrm{~kg}(2.45,4.91$, and $9.82 \mathrm{lb})$. Phenolic guide rings were bonded around both ends of each projectile to protect the honed inside diameter of the guide tube from damage. The accelerometer, mounted at the bottom of a recess bored into the projectile, was protected by a cover which was bolted over the recess.

The uniform compression of test samples confined in steel tubes to prevent lateral expansion was used as a simulation of the boundary conditions where polyurethane foam is typically used. The clearance between the 63.5-mm-diameter (2.5-inch) test samples and the inside diameter of the steel tube holding the samples was approximately $0.25 \mathrm{~mm}(0.010 \mathrm{inch})$. During a test, an extension of the test weight with a nominal diameter of $62.99 \mathrm{~mm}$ ( 2.480 inches) entered the specimen tube approximately $6.35 \mathrm{~mm}$ ( $0.25 \mathrm{inch}$ ) before it contacted and began to crush the sample. Foam samples for testing were machined from billets to minimize density variation.

To perform a test, the sample tube was positioned to the rear of the guide tube and secured in place with setscrews. The foam sample was then inserted into the sample tube. Figure 3 shows the sample in place, ready for testing. The actuator firing pressure and the gap that must be set between the sample and the 


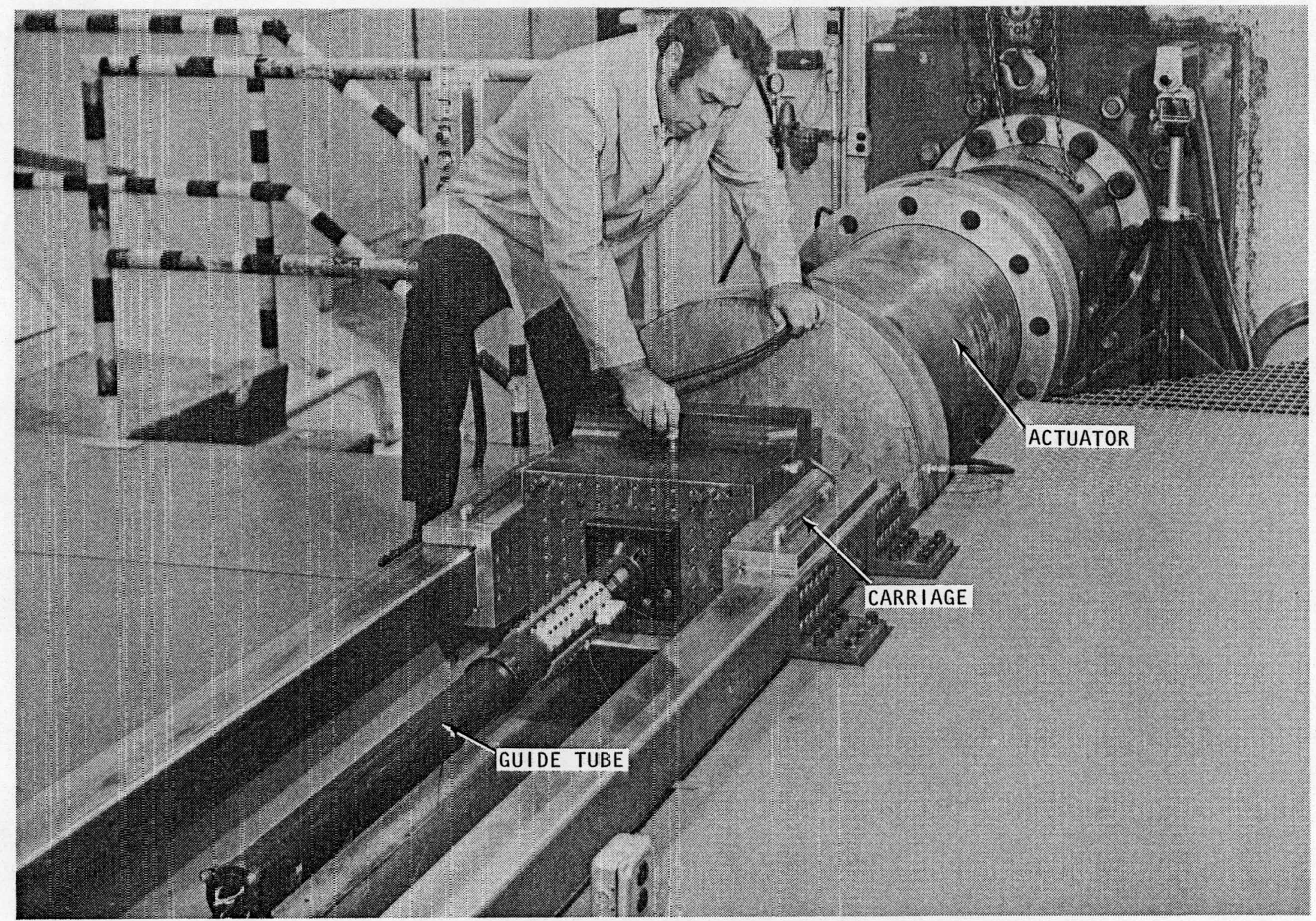

Figure 2. Test Weight Guide Tube Attached to Carriage 


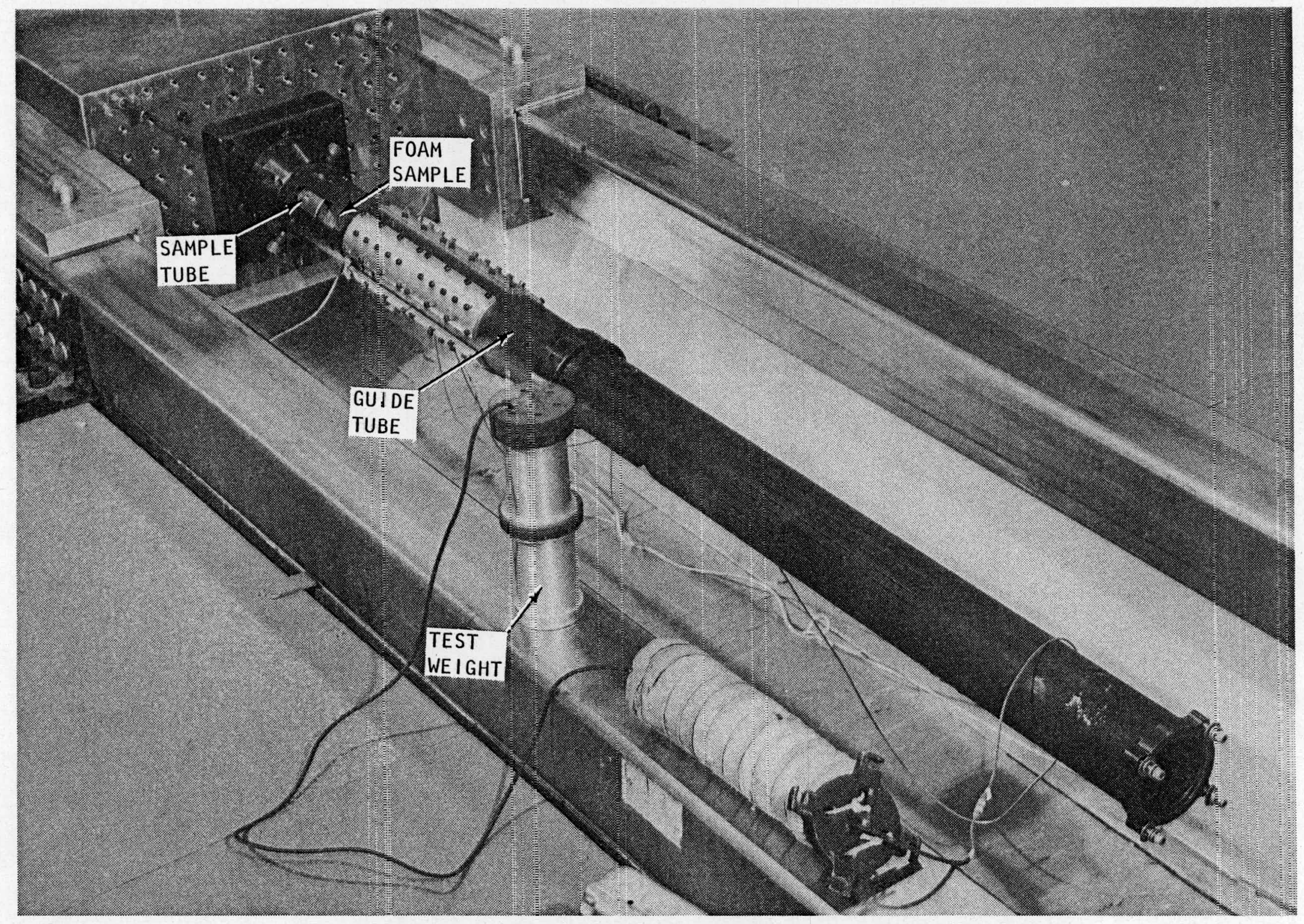

Figure 3. Test Weight With Sample Prepared for Test 
test weight were selected from actuator performance data. The initial gap coincides with the distance over. which the carriage and the guide tube accelerate before reaching impact velocity, which varied from $508 \mathrm{~mm}$ at $15.2 \mathrm{~m} / \mathrm{s}$ ( 20 inches at $50 \mathrm{ft} / \mathrm{sec}$ ) to $914 \mathrm{~mm}$ at $61.0 \mathrm{~m} / \mathrm{s}$ (36 inches at $200 \mathrm{ft} / \mathrm{sec}$ ). Impact of the sample into the test weight occurred just before the carriage separated from the actuator thrust column.

Following impact with the sample, the test weight experienced a post-crush rebound velocity that varied from a few feet per second to $40 \mathrm{ft} / \mathrm{sec}(12 \mathrm{~m} / \mathrm{s})$. Ensolite rubber tied to the guide tube end cap cushioned the impact of the projectile when it reached the end of the tube. A braking system built into the carriage arrested its motion within $15.2 \mathrm{~m}$ (50 feet).

Instrumentation

Velocity Measurement

Magnetic sensors used to measure impact velocity were installed through the wall of the guide tube. Travel time of the test weight was measured between four sensors spaced $25.4 \mathrm{~mm}$ ( 1 inch) apart. A 1.59-mm-thick (1/16 inch) steel ring, fastened to the test weight, caused a voltage output from each sensor during the motion of the guide tube prior to impact. The voltage pulses, along with 1 -millisecond time marks, were recorded on magnetic tape at $3.048 \mathrm{~m} / \mathrm{s}$ (120 inches/second). These were later played back at a speed of $47.62 \mathrm{~mm} / \mathrm{s}$ (1-7/8. inches/second) on to an oscillograph record from which impact velocity calculations were made. Typical signals from an oscillograph record with a velocity calculation are shown in Figure 4. The calculation was generally within 5 percent of the desired test velocity. The total range of uncertainty in a calculated value for impact velocity is 3 percent.

A Kistler Model $805 \mathrm{~A}$ accelerometer, with a $100,000-g$ capability and a $60 \mathrm{kHz}$ resonant frequency, was selected for the tests. The block diagram in Figure 5 illustrates the instrumentation system employed to measure the acceleration. of the test weight. The acceleration trace as well as the magnetic-pickup outputs and the 1-millisecond time marks were recorded on magnetic tape at a speed of $3.048 \mathrm{~m} / \mathrm{s}$ (120 inches/second). Data records were obtained by playing the data back at $47.62 \mathrm{~m} / \mathrm{s}(1-7 / 8$ inches/second) into a Honeywell oscillograph. Typical data recorded from an impact test are shown in Figure 6. After reviewing the data, acceleration traces to be digitized were selected and scaled for playback into a Biomation Model 810 transient recorder at $3.048 \mathrm{~m} / \mathrm{s}$ (120 inches/second). The transient recorder converted the data into digital form and stored it in an internal memory system. The digital data then were punched on paper tape in standard ASCII code. 

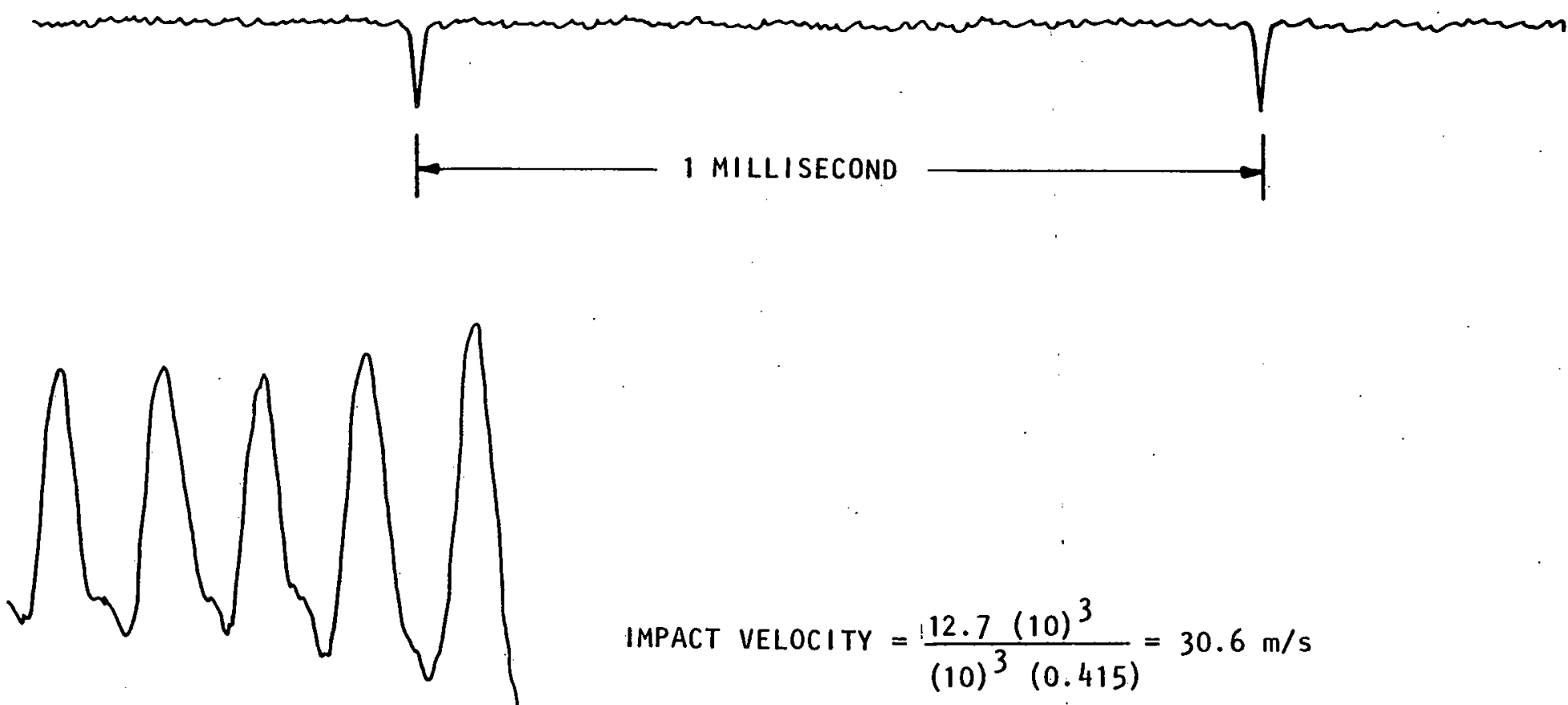

START OF SHOCK PULSE $\longrightarrow$

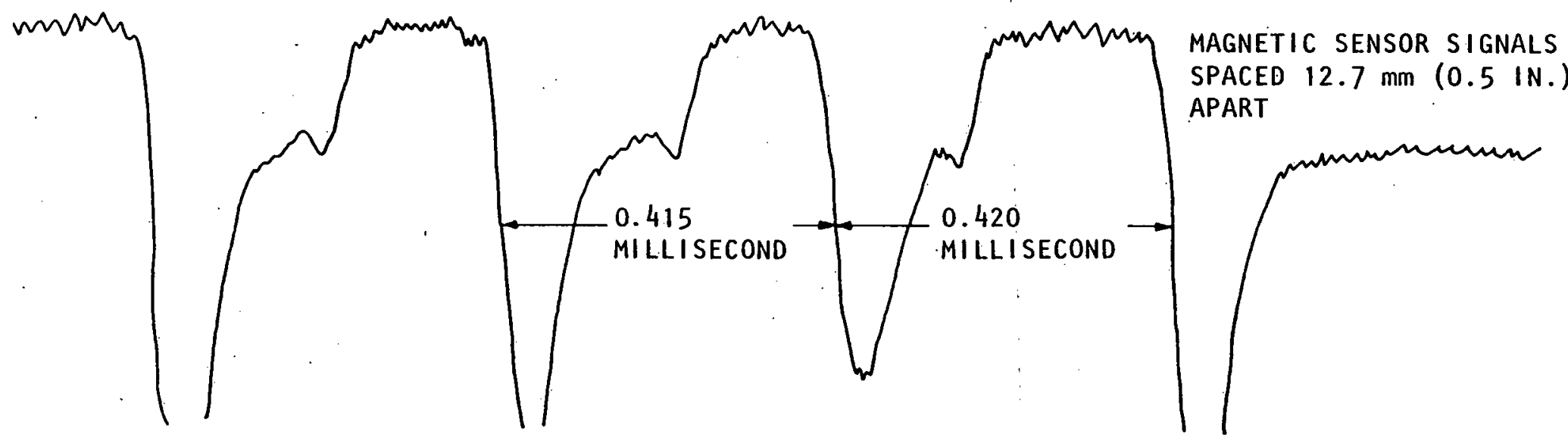

Figure 4. Velocity Calculation From Typical Output of Magnetic Sensors (Tracing) 
MAGNETIC SENSORS

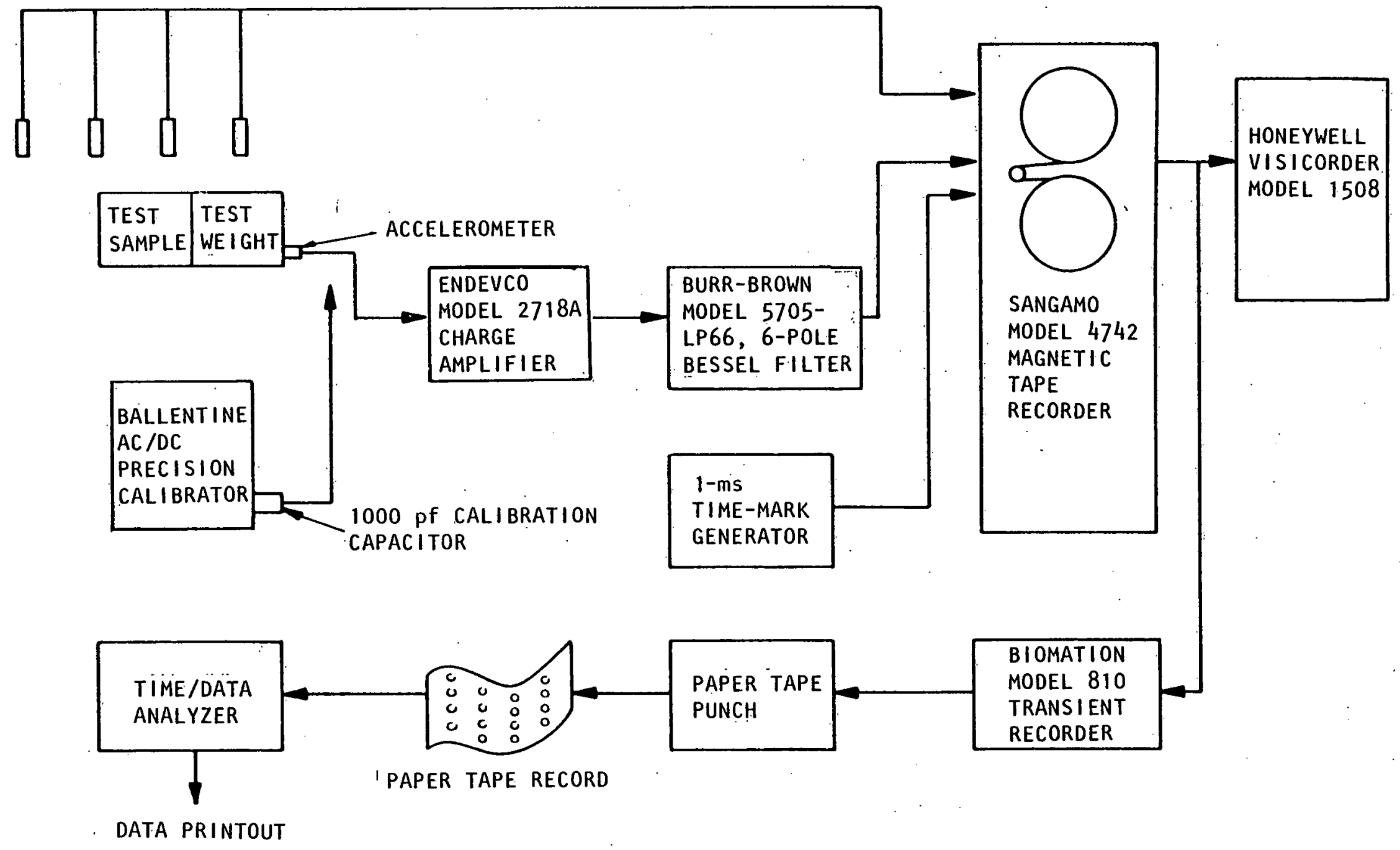

Figure 5. Block Diagram of Instrumentation System 


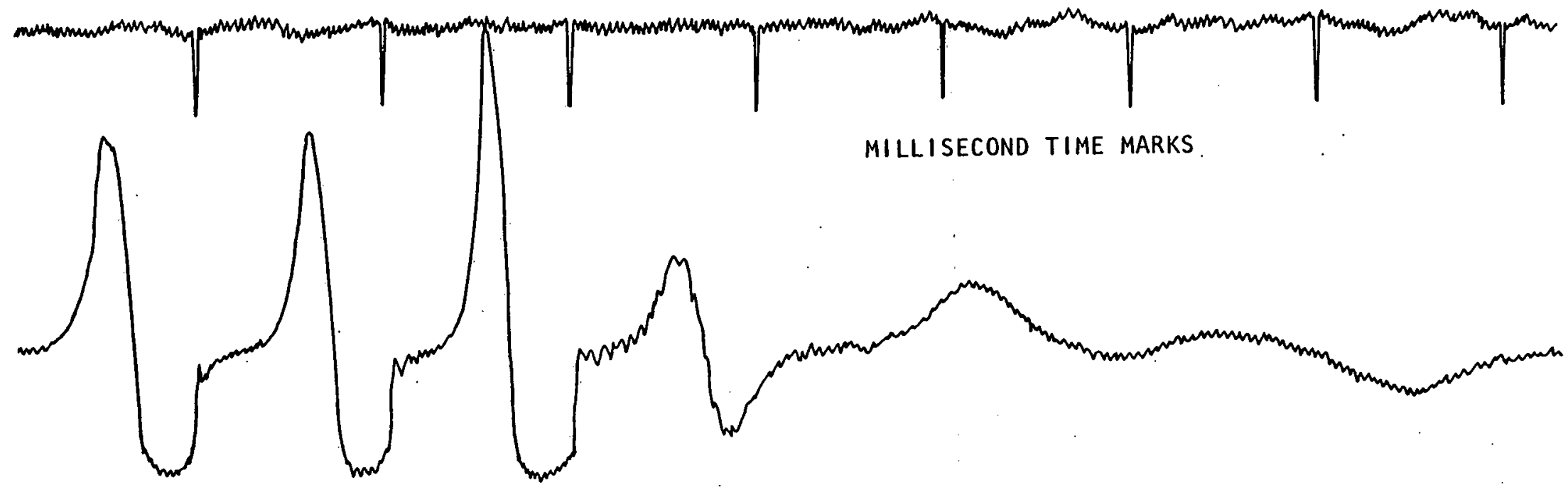

MAGNETIC SENSOR SIGNALS

SPACED $12.7 \mathrm{~mm}(0.5 \mathrm{IN}$.

APART

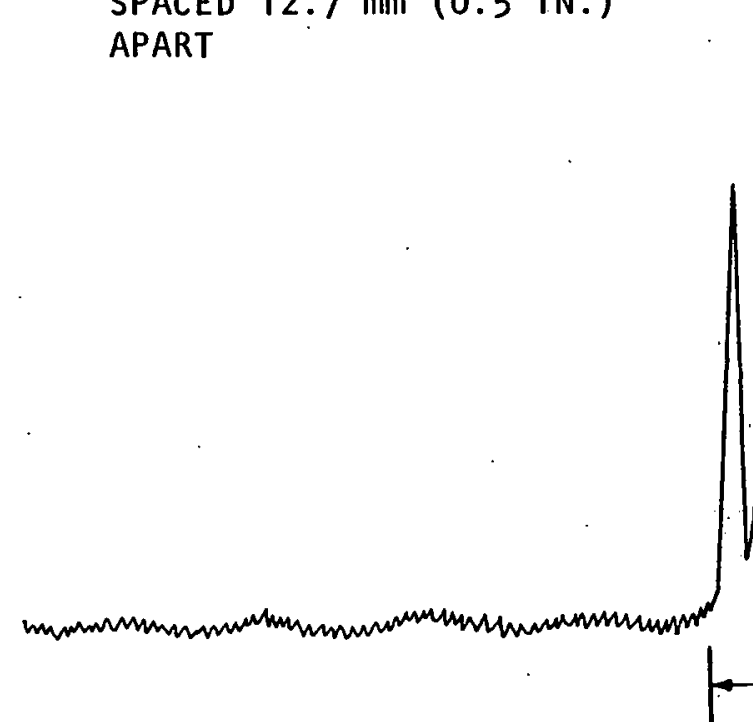

570-g PEAK-FAIRED ACCELERATION

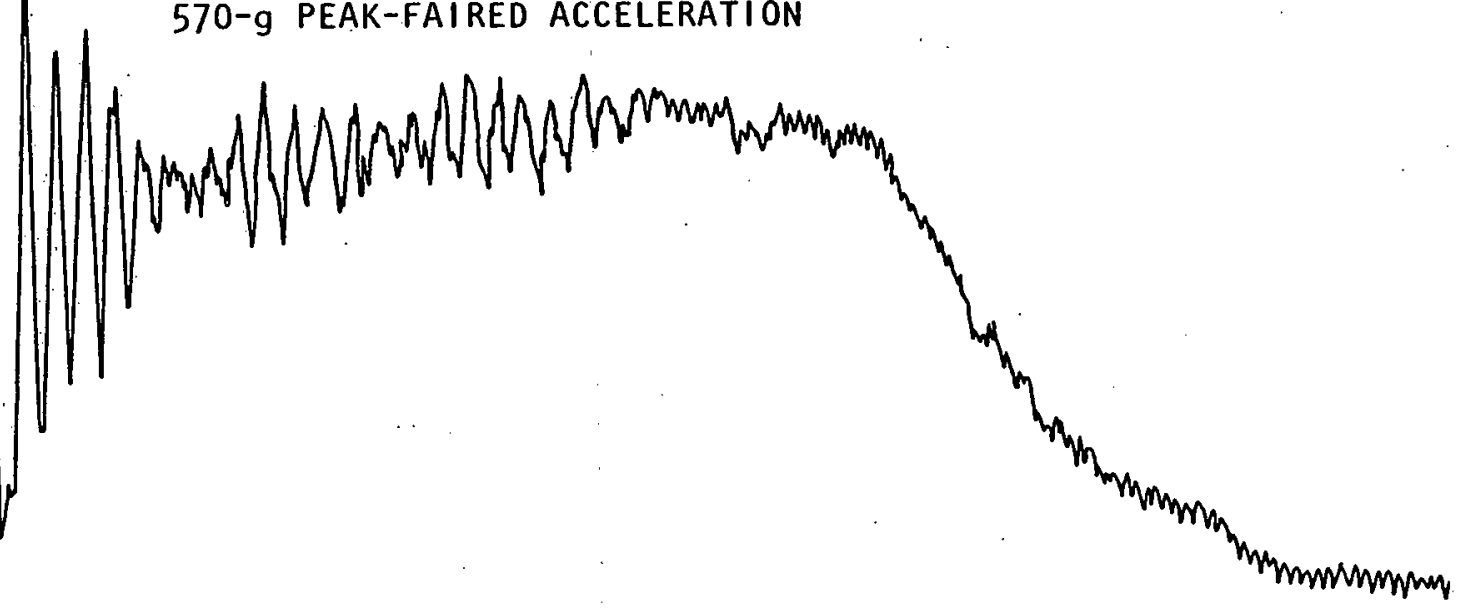

5.2 MILLISECONDS

Figure 6. Typical Oscillograph Record of Impact Test (Tracing) 


\section{Data Reduction}

The paper tape recorder of a test was entered into a time-data, time-series analyzer. The acceleration record was faired by first using a three-point smoothing algorithm. The program routine selected the baseline and limits of integration. The above information was displayed, giving the operator an opportunity to correct any unsatisfactory parameters before continuing. The program routine then determined velocity, sample deflection, and dynamic force versus time along with values for percent rebound, and energy absorbing efficiencies.

Energy-Absorption Efficiency

Energy-absorption efficiency is defined as the ratio of the energy absorbed per unit volume by the test material to the energy absorbed by an ideal material. An ideal energy-absorbing material is one which deflects at a constant stress, thus providing a constant deceleration for 100 percent of its thickness. The kinetic energy absorbed by a materlal bears the following relationship to the energy absorbed by an ideal material.

$M V_{i}^{2} / 2=M d_{m} h$,

where

$M=$ mass of impacting object,

$\mathrm{v}_{i}=$ impact velocity,

$\mathrm{d}_{\mathrm{m}}=$ maximum deceleration,

$\mathrm{h}=$ material thickness, and

$M d_{m}=$ maximum decelerating force.

By definition, the energy-aborbing efficiency ( $K$ ) exhibited by a test material can be represented by the following equation:

$\mathrm{K}=\mathrm{V}_{\mathrm{i}}^{2} / 2 \mathrm{hd}_{\mathrm{m}}$

where, for an ideal material,

$\mathrm{K}=1$.

The conclusion, therefore, can be reached that when $V_{i}$ is low, the impact energy will probably be small in relation to the stiffness of the foam. There will be little compression; and $K$ 
will, therefore, be low. If $V_{i}$ is too high, the impact energy will be large in relation to the stiffness of the foam; the critical strain will be exceeded (which will rapidly increase $\left.d_{m}\right)$; and $\mathrm{K}$ again will be low. At some intermediate value of $V_{i}$, $\mathrm{K}$ will exhibit a maximum. Values for energy-absorbing efficiency (K) are reported in Appendix A for each impact condition. The specific energy at each impact condition is provided in Table 1.

\section{Data Presentation}

When crushed, rigid polyurethane foam samples generally experience some degree of recovery (rebound). This post-impact recovery of the material is referred to as restituted strain. The rebound velocity of the test weight expressed as a percentage of the impact velocity, and the deflection of the test weight, before and after rebound, were determined for each impact condition. The numerical data for these conditions are provided in Appendix A.

Appendix B provides a graphical presentation of acceleration versus displacement for each test condition. In addition, a static compressive strength plot is superimposed on each set of dynamic plots to allow simultaneous evaluation of static and dynamic response. Samples for static testing were prepared in a manner identical to the dynamic testing samples and were crushed at a rate of $0.42 \mathrm{~mm} / \mathrm{s}$ ( 1 inch/minute), using conventional loaddeflection measuring equipment.

To allow effective use of the data in this report, a study was made to evaluate the effect of input errors on the accuracy of a typical test. A square wave best represents the acceleration time-trace obtained during the crush of a foam sample. Input errors were substituted into equations developed from the motion-time equations for a square wave pulse to indicate the total range of uncertainty for a typical impact test. It can be generally concluded that the accuracy of the output data is within \pm 10.5 percent. This information is detailed in Table 2.

\section{ACCOMPLISHMENTS}

Test techniques and the required equipment have been developed for use in evaluating structural support materials. These techniques have been used to evaluate six commonly used polyurethane foam systems, and the subsequent data have been published for reference.

\section{FUTURE WORK}

All planned activities have been completed. Additional studies may be performed by other endeavors as the need arises. 
Table 1. Energy Related to Test Weights and Impact Velocities

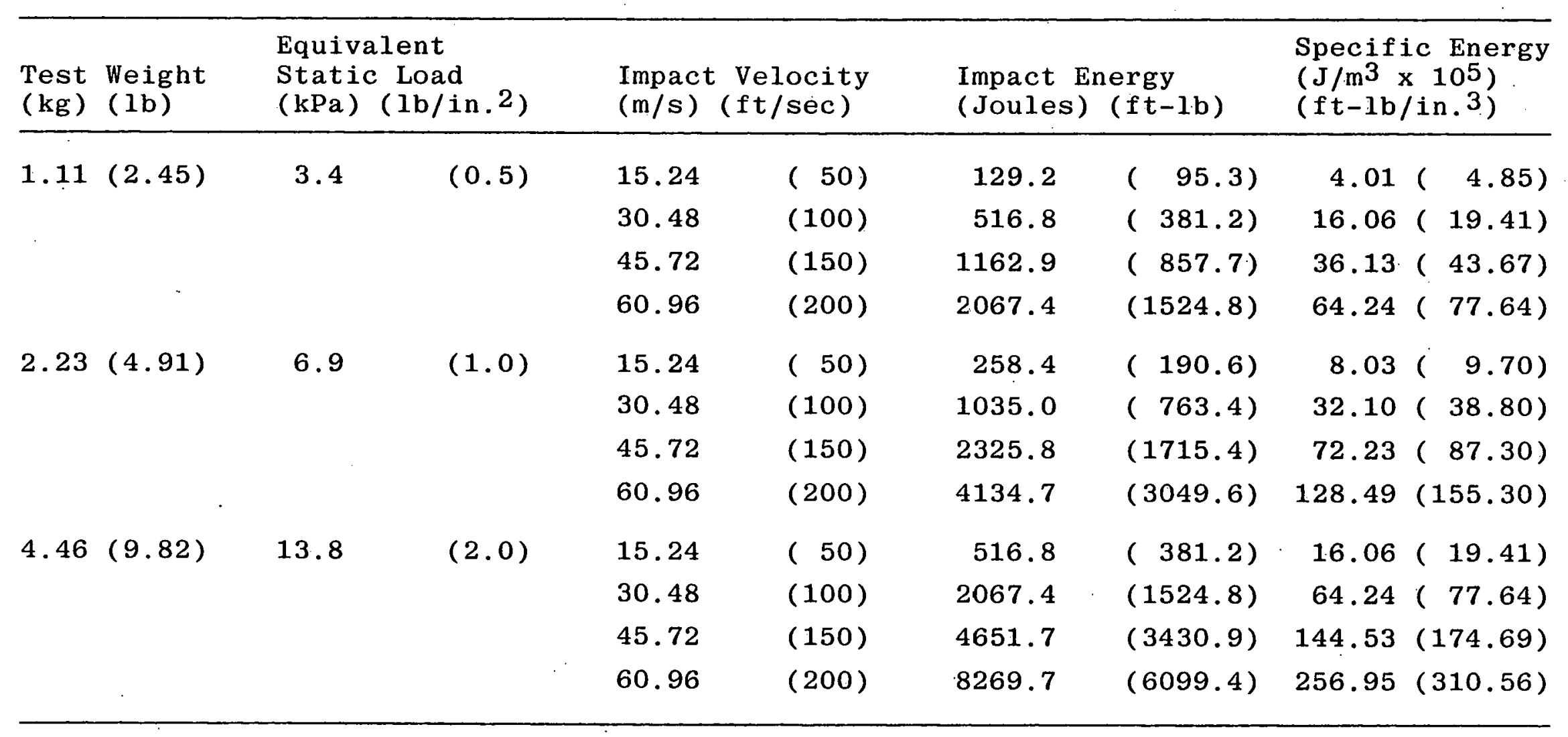


Table 2. Effect of Input-Parameter Errors Upon Accuracy of Output Data

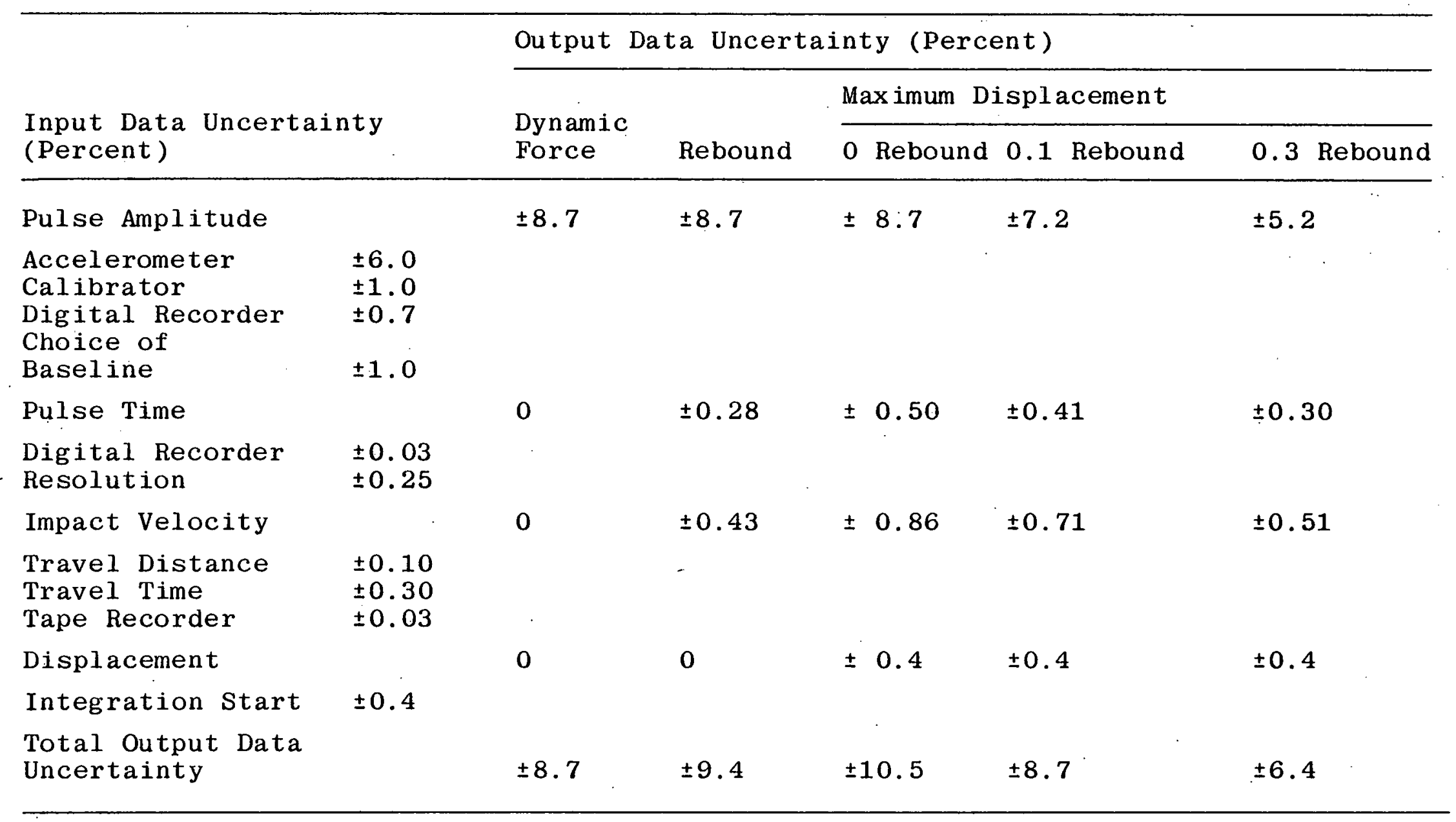


Appendix A

IMPACT DATA TABLES 
Table A-1: Impact Data for CPR 1024

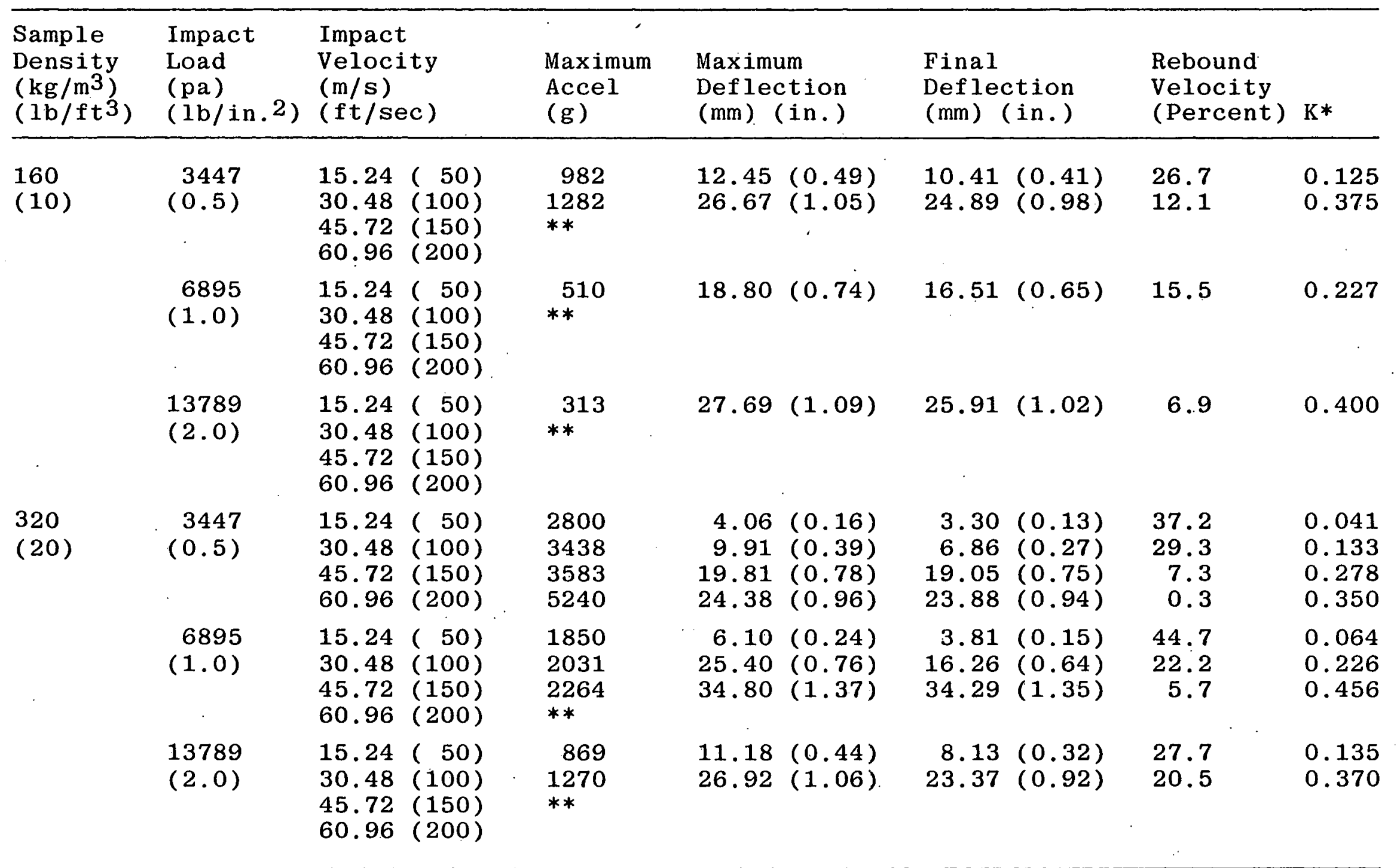

$* \mathrm{~K}=$ Energy Absorption Efficiency

**Critical Strain Exceeded 
Table A-2. Impact Data for CPR 1040

\begin{tabular}{|c|c|c|c|c|c|c|c|}
\hline $\begin{array}{l}\text { Sample } \\
\text { Density } \\
\left(\mathrm{kg} / \mathrm{m}^{3}\right) \\
\left(1 \mathrm{~b} / \mathrm{ft}^{3}\right)\end{array}$ & $\begin{array}{l}\text { Impact } \\
\text { Load } \\
(\text { pa) } \\
(1 \mathrm{l} / \mathrm{in} .2)\end{array}$ & $\begin{array}{l}\text { Impact } \\
\text { Velocity } \\
(\mathrm{m} / \mathrm{s}) \\
(\mathrm{ft} / \mathrm{sec})\end{array}$ & $\begin{array}{l}\text { Maximum } \\
\text { Accel } \\
(\mathrm{g})\end{array}$ & $\begin{array}{l}\text { Maximum } \\
\text { Deflection } \\
(\mathrm{mm})(\text { in.) }\end{array}$ & $\begin{array}{l}\text { Final } \\
\text { Deflection } \\
(\mathrm{mm}) \text { (in.) }\end{array}$ & $\begin{array}{l}\text { Rebound } \\
\text { Velocity } \\
\text { (Percent) }\end{array}$ & $\mathrm{K}^{*}$ \\
\hline \multirow[t]{2}{*}{$\begin{array}{l}160 \\
(10)\end{array}$} & $\begin{array}{l}3447 \\
(0.5)\end{array}$ & $\begin{array}{l}15.24 \quad(50) \\
30.48,(100) \\
45.72(150) \\
60.96(200)\end{array}$ & $\begin{array}{l}680 \\
1372 \\
* *\end{array}$ & $\begin{array}{l}14.22(0.56) \\
22.10(0.87)\end{array}$ & $\begin{array}{l}14.22(0.56) \\
19.56(0.77)\end{array}$ & $\begin{array}{l}0 \\
15.2\end{array}$ & $\begin{array}{l}0.139 \\
0.326\end{array}$ \\
\hline & $\begin{array}{l}13789 \\
(2.0)\end{array}$ & $\begin{array}{l}15.24(50) \\
30.48(100) \\
45.72(150) \\
60.96(200)\end{array}$ & $\begin{array}{l}368 \\
* *\end{array}$ & $23.14(0.91)$ & $21.34(0.84)$ & 11.3 & 0.327 \\
\hline \multirow{2}{*}{$\begin{array}{l}320 \\
(20)\end{array}$} & $\begin{array}{r}6895 \\
(1.0)\end{array}$ & $\begin{array}{l}15.24 \quad(50) \\
30.48(100) \\
45.72(150) \\
60.96(200)\end{array}$ & $\begin{array}{l}2175 \\
2524 \\
2857 \\
* *\end{array}$ & $\begin{array}{r}6.60(0.26) \\
15.24(0.60) \\
26.67(1.05)\end{array}$ & $\begin{array}{r}3.81(0.15) \\
13.46(0.53) \\
26.67(1.05)\end{array}$ & $\begin{array}{l}55.2 \\
16.2 \\
0\end{array}$ & $\begin{array}{l}0.053 \\
0.186 \\
0.374\end{array}$ \\
\hline & $\begin{array}{l}13789 \\
(2.0)\end{array}$ & 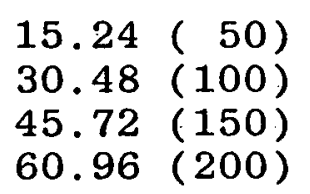 & $\begin{array}{l}1030 \\
1736 \\
* *\end{array}$ & $\begin{array}{l}1.1 .43(0.45) \\
20.57(0.81)\end{array}$ & $\begin{array}{l}11.18(0.44) \\
16.76(0.66)\end{array}$ & $\begin{array}{r}4.0 \\
25.4\end{array}$ & $\begin{array}{l}0.110 \\
0.264\end{array}$ \\
\hline
\end{tabular}

$* \mathrm{~K}=$ Energy Absorption Efficiency

**Critical Strain Exceeded 
Table A-3. Impact Data for BC 1200 Series

\begin{tabular}{|c|c|c|c|c|c|c|c|}
\hline $\begin{array}{l}\text { Sample } \\
\text { Density } \\
\left(\mathrm{kg} / \mathrm{m}^{3}\right) \\
\left(\mathrm{lb} / \mathrm{ft}^{3}\right)\end{array}$ & $\begin{array}{l}\text { Impact } \\
\text { Load } \\
(\text { pa }) \\
\left(1 \mathrm{~b} / \mathrm{in.}{ }^{2}\right)\end{array}$ & $\begin{array}{l}\text { Impact } \\
\text { Velocity } \\
(\mathrm{m} / \mathrm{s}) \\
(\mathrm{ft} / \mathrm{sec})\end{array}$ & $\begin{array}{l}\text { Maximum } \\
\text { Accel } \\
\text { (g) }\end{array}$ & $\begin{array}{l}\text { Maximum } \\
\text { Deflection } \\
(\mathrm{mm}) \text { (in..) }\end{array}$ & $\begin{array}{l}\text { Final } \\
\text { Deflection } \\
(\mathrm{mm}) \text { (in.) }\end{array}$ & $\begin{array}{l}\text { Rebound } \\
\text { Velocity } \\
\text { (percent) }\end{array}$ & $\mathrm{K} *$ \\
\hline \multirow[t]{3}{*}{$\begin{array}{l}160 \\
(10)\end{array}$} & $\begin{array}{r}3447 \\
(0.5)\end{array}$ & $\begin{array}{ll}15.24 & (50) \\
30.48 & (100) \\
45.72 & (150) \\
60.96 & (200)\end{array}$ & $\begin{array}{l}1714 \\
1967 \\
2627\end{array}$ & $\begin{array}{r}3.38(0.33) \\
20.83(0.82) \\
34.80(1.37)\end{array}$ & $\begin{array}{r}6.60(0.26) \\
18.29(0.72) \\
33.02(1.30)\end{array}$ & $\begin{array}{l}89.7 \\
18.8 \\
13.4\end{array}$ & $\begin{array}{l}0.063 \\
0.233 \\
0.407\end{array}$ \\
\hline & $\begin{array}{l}6895 \\
(1.0)\end{array}$ & $\begin{array}{ll}15.24 & (50) \\
30.48 & (100) \\
45.72 & (150) \\
60.96 & (200)\end{array}$ & $\begin{array}{r}880 \\
1174 \\
* *\end{array}$ & $\begin{array}{ll}11.94 & (0.47) \\
28.96 & (1.14)\end{array}$ & $\begin{array}{l}10.67(0.42) \\
27.43(1.08)\end{array}$ & $\begin{array}{l}20.4 \\
12.2\end{array}$ & $\begin{array}{l}0.138 \\
0.381\end{array}$ \\
\hline & $\begin{array}{l}13789 \\
(2.0)\end{array}$ & $\begin{array}{ll}15.24 & (50) \\
30.48 & (100) \\
45.72 & (150) \\
60.96 & (200)\end{array}$ & $*^{508}$ & $16.51(0.65)$ & $15.24(0.60)$ & 14.7 & 0.234 \\
\hline \multirow[t]{2}{*}{$\begin{array}{l}320 \\
(20)\end{array}$} & $\begin{array}{r}3447 \\
(0.5)\end{array}$ & $\begin{array}{ll}15.24 & (50) \\
30.48 & (100) \\
45.72 & (150) \\
60.96 & (200)\end{array}$ & $\begin{array}{l}4300 \\
4600 \\
5500 \\
6537\end{array}$ & $\begin{array}{r}4.83(0.19) \\
7.37(0.29) \\
18.03(0.71) \\
21.34(0.84)\end{array}$ & $\begin{array}{r}2.03(0.08) \\
5.59(0.22) \\
14.99(0.59) \\
17.78(0.70)\end{array}$ & $\begin{array}{l}71.1 \\
31.5 \\
22.5 \\
19.1\end{array}$ & $\begin{array}{l}0.030 \\
0.102 \\
0.187 \\
0.280\end{array}$ \\
\hline & $\begin{array}{r}6895 \\
(1.0)\end{array}$ & $\begin{array}{ll}15.24 & (50) \\
30.48 & (100) \\
45.72 & (150) \\
60.96 & (200)\end{array}$ & $\begin{array}{l}2287 \\
2891 \\
* *\end{array}$ & $\begin{array}{r}6.86(0.27) \\
11.43(0.45)\end{array}$ & $\begin{array}{l}5.33(0.21) \\
8.89(0.35)\end{array}$ & $\begin{array}{l}36.9 \\
29.7\end{array}$ & $\begin{array}{l}0.053 \\
0.155\end{array}$ \\
\hline . & $\begin{array}{l}13789 \\
(2.0)\end{array}$ & $\begin{array}{ll}15.24 & (50) \\
30.48 & (100) \\
45.72 & (150) \\
60.96 & (200)\end{array}$ & $\begin{array}{l}1199 \\
1687 \\
* *\end{array}$ & $\begin{array}{r}8.34(0.34) \\
21.34(0.84)\end{array}$ & $\begin{array}{r}7.37(0.29) \\
19.30(0.76)\end{array}$ & $\begin{array}{l}15.9 \\
16.4\end{array}$ & $\begin{array}{l}0.093 \\
0.277\end{array}$ \\
\hline
\end{tabular}


Table A-3 Continued. Impact Data for BC 1200 Series

\begin{tabular}{|c|c|c|c|c|c|c|c|}
\hline $\begin{array}{l}\text { Sample } \\
\text { Density } \\
\left(\mathrm{kg} / \mathrm{m}^{3}\right) \\
\left(1 \mathrm{~b} / \mathrm{ft}^{3}\right)\end{array}$ & $\begin{array}{l}\text { Impact } \\
\text { Load } \\
(\text { pa) } \\
\left(1 \mathrm{~b} / \text { in }^{2}\right)\end{array}$ & $\begin{array}{l}\text { Impact } \\
\text { Velocity } \\
(\mathrm{m} / \mathrm{s}) \\
(\mathrm{ft} / \mathrm{sec})\end{array}$ & $\begin{array}{l}\text { Maximum } \\
\text { Accel } \\
(\mathrm{g})\end{array}$ & $\begin{array}{l}\text { Maximum } \\
\text { Deflection } \\
(\mathrm{mm}) \text { (in.) }\end{array}$ & $\begin{array}{l}\text { Final } \\
\text { Deflection } \\
(\mathrm{mm}) \text { (in:) }\end{array}$ & $\begin{array}{l}\text { Rebound } \\
\text { Velocity } \\
\text { (Percent) }\end{array}$ & $\mathrm{K} *$ \\
\hline \multirow[t]{4}{*}{$\begin{array}{l}480 \\
(30)\end{array}$} & $\begin{array}{r}3447 \\
(0.5)\end{array}$ & $\begin{array}{ll}15.24 & (50) \\
30.48 & (100) \\
45.72 & (150) \\
60.96 & (200)\end{array}$ & $\begin{array}{r}6600 \\
11000\end{array}$ & $\begin{array}{l}3.05(0.12) \\
8.38(0.33)\end{array}$ & $\begin{array}{l}1.02(0.04) \\
5.59(0.22)\end{array}$ & $\begin{array}{l}84.1 \\
43.6\end{array}$ & $\begin{array}{l}0.017 \\
0.90\end{array}$ \\
\hline & $\begin{array}{l}13789 \\
(2.0)\end{array}$ & $\begin{array}{ll}15.24 & (50) \\
30.48 & (100) \\
45.72 & (150) \\
60.96 & (200)\end{array}$ & $\begin{array}{l}2778 \\
3248 \\
4024 \\
* *\end{array}$ & $\begin{aligned} 4.83 & (0.19) \\
15.24 & (0.60 \\
21.59 & (0.85)\end{aligned}$ & $\begin{array}{r}3.56(0.14) \\
14.22(0.56) \\
19.56(0.77)\end{array}$ & $\begin{array}{r}36.3 \\
9.0 \\
16.9\end{array}$ & $\begin{array}{l}0.042 \\
0.138 \\
0.266\end{array}$ \\
\hline & $\begin{array}{r}6895 \\
(1.0)\end{array}$ & $\begin{array}{l}15.24 \quad(50) \\
30.48(100) \\
45.72(150) \\
60.96(200)\end{array}$ & & & & & . \\
\hline & $\begin{array}{l}13789 \\
(2.0)\end{array}$ & $\begin{array}{ll}15.24 & (50) \\
30.48 & (100) \\
45.72 & (150) \\
60.96 & (200)\end{array}$ & & & & & \\
\hline
\end{tabular}

$* K=$ Energy Absorption Efficiency 
Table A-4. Impact Data for BKC 4003

\begin{tabular}{|c|c|c|c|c|c|c|c|}
\hline $\begin{array}{l}\text { Sample } \\
\text { Density } \\
\left(\mathrm{kg} / \mathrm{m}^{3}\right) \\
\left(1 \mathrm{~b} / \mathrm{ft}^{3}\right)\end{array}$ & $\begin{array}{l}\text { Impact } \\
\text { Load } \\
(\text { pa) } \\
(1 b / \text { in. } 2)\end{array}$ & $\begin{array}{l}\text { Impact } \\
\text { Velocity } \\
(\mathrm{m} / \mathrm{s}) \\
(\mathrm{ft} / \mathrm{sec})\end{array}$ & $\begin{array}{l}\text { Maximum } \\
\text { Accel } \\
\text { (g) }\end{array}$ & $\begin{array}{l}\text { Maximum } \\
\text { Deflection } \\
(\mathrm{mm}) \text { (in.) }\end{array}$ & $\begin{array}{l}\text { Final } \\
\text { Deflection } \\
(\mathrm{mm}) \text { (in.) }\end{array}$ & $\begin{array}{l}\text { Rebound } \\
\text { Velocity } \\
\text { (Percent) }\end{array}$ & $\mathrm{K}^{*}$ \\
\hline \multirow[t]{3}{*}{$\begin{array}{l}160 \\
(10)\end{array}$} & $\begin{array}{r}3447 \\
(0.5)\end{array}$ & $\begin{array}{l}15.24(50) \\
30.48(100) \\
45.72(150) \\
60.96(200)\end{array}$ & $\begin{array}{l}842 \\
1195 \\
* *\end{array}$ & $\begin{array}{l}12.70(0.50) \\
30.23(1.19)\end{array}$ & $\begin{array}{ll}11.94 & (0.47) \\
25.91 & (1.02)\end{array}$ & $\begin{array}{l}13.8 \\
20.7\end{array}$ & $\begin{array}{l}0.144 \\
0.388\end{array}$ \\
\hline & $\begin{array}{l}6895 \\
(1.0)\end{array}$ & $\begin{array}{ll}15.24 & (50) \\
30.48 & (100) \\
45.72 & (150) \\
60.96 & (200)\end{array}$ & $\begin{array}{l}477 \\
* *\end{array}$ & $19.30(0.76)$ & $15.75(0.62)$ & 23.6 & 0.255 \\
\hline & $\begin{array}{l}13789 \\
(2.0)\end{array}$ & $\begin{array}{ll}15.24 & (50) \\
30.48 & (100) \\
45.72 & (150) \\
60.96 & (200)\end{array}$ & $\begin{array}{l}281 \\
* *\end{array}$ & $22.10(0.87)$ & $\begin{array}{c}22.10(0.87) \\
\vdots\end{array}$ & 11.1 & 0.406 \\
\hline \multirow[t]{3}{*}{$\begin{array}{l}320 \\
(20)\end{array}$} & $\begin{array}{r}3447 \\
(0.5)\end{array}$ & $\begin{array}{ll}15.24 & (50) \\
30.48 & (100) \\
45.72 & (150) \\
60.96 & (200)\end{array}$ & $\begin{array}{l}3117 \\
3378 \\
4144\end{array}$ & $\begin{array}{r}5.84(0.23) \\
11.18(0.44) \\
18.03(0.71)\end{array}$ & $\begin{aligned} 2.03 & (0.08) \\
7.37 & (0.29) \\
15.49 & (0.61)\end{aligned}$ & $\begin{array}{l}68.2 \\
32.7 \\
24.6\end{array}$ & $\begin{array}{l}0.039 \\
0.136 \\
0.249\end{array}$ \\
\hline & $\begin{array}{l}6895 \\
(1.0)\end{array}$ & $\begin{array}{ll}15.24 & (50) \\
30.48 & (100) \\
45.72 & (150) \\
60.96 & (200)\end{array}$ & $\begin{array}{l}1345 \\
2042 \\
3551\end{array}$ & $\begin{array}{rr}8.38 & (0.33) \\
17.53 & (0.69) \\
32.26 & (1.27)\end{array}$ & $\begin{array}{r}8.38(0.33) \\
14.73(0.58) \\
28.70(1.13)\end{array}$ & $\begin{array}{r}9.0 \\
22.3 \\
22.4\end{array}$ & $\begin{array}{l}0.083 \\
0.230 \\
0.307\end{array}$ \\
\hline & $\begin{array}{l}13789 \\
(2.0)\end{array}$ & $\begin{array}{ll}15.24 & (50) \\
30.48 & (100) \\
45.72 & (150) \\
60.96 & (200)\end{array}$ & $\begin{array}{l}1149 \\
1282 \\
* *\end{array}$ & $\begin{array}{r}8.64(0.34) \\
26.42(1.04)\end{array}$ & $\begin{array}{r}6.86(0.27) \\
24.13(0.95)\end{array}$ & $\begin{array}{l}24.5 \\
15.7\end{array}$ & $\begin{array}{l}0.109 \\
0.357\end{array}$ \\
\hline
\end{tabular}


Table A-4 Continued. Impact Data for BKC 4003

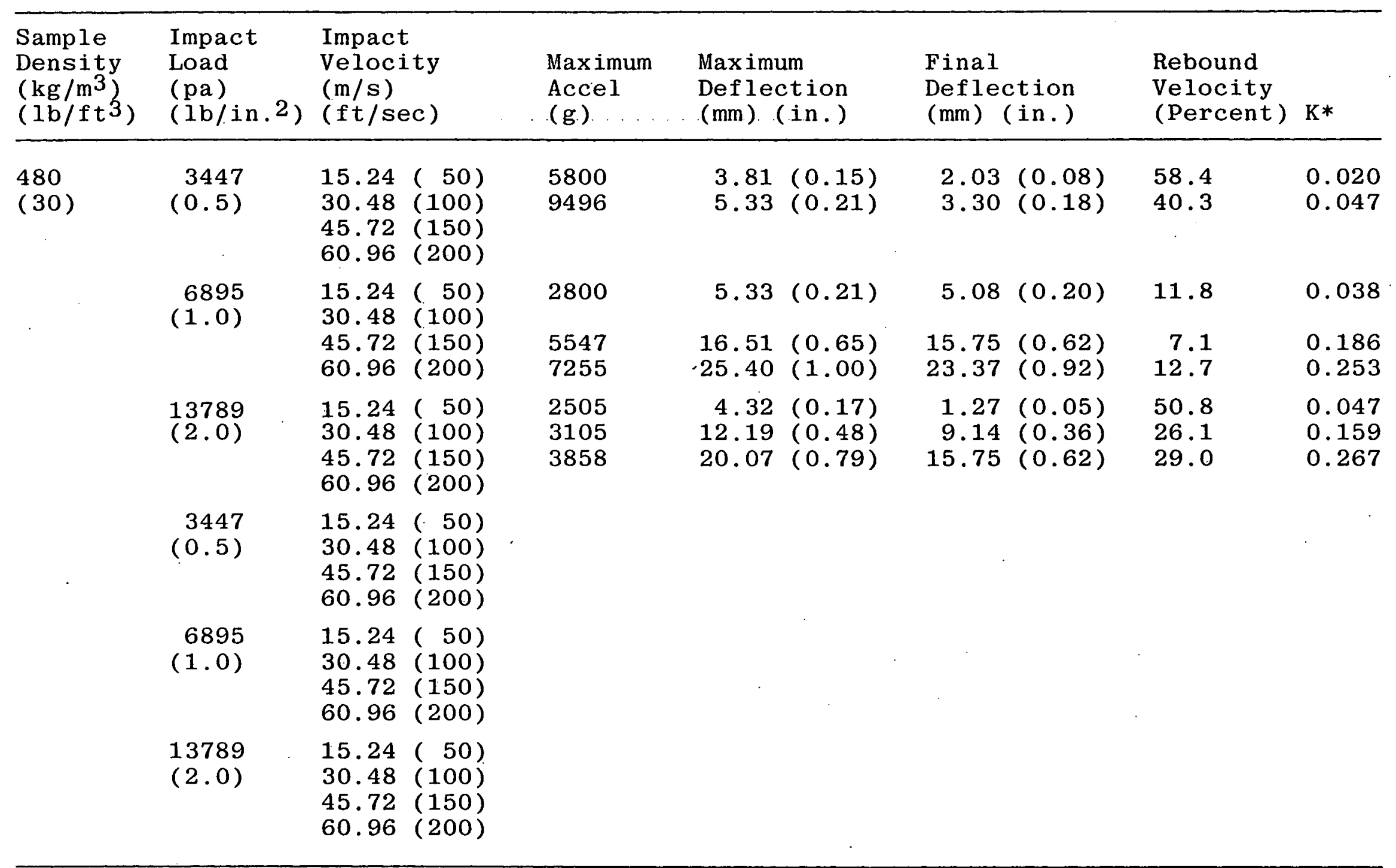

$* \mathrm{~K}=$ Energy Absorption Efficiency **Critical Strain Exceeded 
Table A-5. Impact Data for BKC 6003

\begin{tabular}{|c|c|c|c|c|c|c|c|}
\hline $\begin{array}{l}\text { Sample } \\
\text { Density } \\
\left(\mathrm{kg} / \mathrm{m}^{3}\right) \\
\left(\mathrm{lb} / \mathrm{ft}^{3}\right)\end{array}$ & $\begin{array}{l}\text { Impact } \\
\text { Load } \\
(\text { pa) } \\
(1 \mathrm{~b} / \text { in. } 2)\end{array}$ & $\begin{array}{l}\text { Impact } \\
\text { Velocity } \\
(\mathrm{m} / \mathrm{s}) \\
(\mathrm{ft} / \mathrm{sec})\end{array}$ & $\begin{array}{l}\text { Maximum } \\
\text { Accel } \\
\text { (g) } \ldots\end{array}$ & $\begin{array}{l}\text { Maximum } \\
\text { Deflection } \\
(\mathrm{mm}) \text { (in..) }\end{array}$ & $\begin{array}{l}\text { Final } \\
\text { Deflection } \\
(\mathrm{mm}) \text { (in.) }\end{array}$ & $\begin{array}{l}\text { Rebound } \\
\text { Velocity } \\
\text { (Percent) }\end{array}$ & $\mathrm{K}^{*}$ \\
\hline \multirow[t]{3}{*}{$\begin{array}{l}160 \\
(10)\end{array}$} & $\begin{array}{r}3447 \\
(0.5)\end{array}$ & $\begin{array}{ll}15.24 & (50) \\
30.48 & (100) \\
45.72 & (150) \\
60.96 & (200)\end{array}$ & $\begin{array}{l}1020 \\
1351 \\
* *\end{array}$ & $\begin{array}{rr}9.91 & (0.39) \\
28.70 & (1.13)\end{array}$ & $\begin{array}{rr}8.38 & (0.33) \\
24.89 & (0.98)\end{array}$ & $\begin{array}{l}23.2 \\
14.2\end{array}$ & $\begin{array}{l}0.115 \\
0.356\end{array}$ \\
\hline & $\begin{array}{r}6895 \\
(1.0)\end{array}$ & $\begin{array}{ll}15.24 & (50) \\
30.48 & (100) \\
45.72 & (150) \\
60.96 & (200)\end{array}$ & $\begin{array}{l}585 \\
972 \\
* *\end{array}$ & $\begin{array}{ll}16.26 & (0.64) \\
39.88 & (1.57)\end{array}$ & $\begin{array}{ll}14.22 & (0.56) \\
38.61 & (1.52)\end{array}$ & $\begin{array}{r}16.0 \\
0\end{array}$ & $\begin{array}{l}0.189 \\
0.483\end{array}$ \\
\hline & $\begin{array}{l}13789 \\
(2.0)\end{array}$ & $\begin{array}{ll}15.24 & (50) \\
30.48 & (100) \\
45.72 & (150) \\
60.96 & (200)\end{array}$ & $\begin{array}{l}338 \\
* *\end{array}$ & $25.40(1.00)$ & $25.40(1.00)$ & 0 & 0.374 \\
\hline \multirow[t]{3}{*}{$\begin{array}{l}320 \\
(20)\end{array}$} & $\begin{array}{r}3447 \\
(0.5)\end{array}$ & $\begin{array}{ll}15.24 & (50) \\
30.48 & (100) \\
45.72 & (150) \\
60.96 & (200)\end{array}$ & $\begin{array}{l}3756 \\
4187\end{array}$ & $\begin{array}{l}3.81(0.15) \\
9.65(0.38)\end{array}$ & $\begin{array}{l}2.54(0.10) \\
8.38(0.33)\end{array}$ & $\begin{array}{l}46.3 \\
15.0\end{array}$ & $\begin{array}{l}0.033 \\
0.112\end{array}$ \\
\hline & $\begin{array}{r}6895 \\
(1.0)\end{array}$ & $\begin{array}{ll}15.24 & (50) \\
30.48 & (100) \\
45.72 & (150) \\
60.96 & (200)\end{array}$ & $\begin{array}{l}1086 \\
2676 \\
3754\end{array}$ & $\begin{array}{r}8.89(0.35) \\
16.51(0.65) \\
21.34 \quad(0.84)\end{array}$ & $\begin{array}{r}8.89(0.35) \\
13.97(0.55) \\
10.92(0.43)\end{array}$ & $\begin{array}{r}0 \\
16.6 \\
13.6\end{array}$ & $\begin{array}{l}0.103 \\
0.178 \\
0.275\end{array}$ \\
\hline & $\begin{array}{l}13789 \\
(2.0)\end{array}$ & $\begin{array}{l}15.24 \quad(50) \\
30.48 \\
45.72(100) \\
60.96(150) \\
(200)\end{array}$ & $\begin{array}{l}1178 \\
1790 \\
* *\end{array}$ & $\begin{aligned} 9.14 & (0.36) \\
20.07 & (0.79)\end{aligned}$ & $\begin{array}{r}7.37(0.29) \\
18.80(0.74)\end{array}$ & $\begin{array}{l}24.6 \\
12.0\end{array}$ & $\begin{array}{l}0.102 \\
0.255\end{array}$ \\
\hline
\end{tabular}


Table A-5 Continued. Impact Data for BKC 6003

\begin{tabular}{|c|c|c|c|c|c|c|c|}
\hline $\begin{array}{l}\text { Sample } \\
\text { Density } \\
\left(\mathrm{kg} / \mathrm{m}^{3}\right) \\
\left(1 \mathrm{~b} / \mathrm{ft}^{3}\right)\end{array}$ & $\begin{array}{l}\text { Impact } \\
\text { Load } \\
(\text { pa) } \\
(1 b / \text { in.2) }\end{array}$ & $\begin{array}{l}\text { Impact } \\
\text { Velocity } \\
(\mathrm{m} / \mathrm{s}) \\
(\mathrm{ft} / \mathrm{sec})\end{array}$ & $\begin{array}{l}\text { Maximum } \\
\text { Accel } \\
\text { (g) }\end{array}$ & $\begin{array}{l}\text { Maximum } \\
\text { Deflection } \\
(\mathrm{mm}) \text { (in.) }\end{array}$ & $\begin{array}{l}\text { Final } \\
\text { Deflection } \\
\text { (mm) (in.) }\end{array}$ & $\begin{array}{l}\text { Rebound } \\
\text { Velocity } \\
\text { (Percent) }\end{array}$ & $\mathrm{K} *$ \\
\hline \multirow[t]{6}{*}{$\begin{array}{l}480 \\
(30)\end{array}$} & $\begin{array}{r}3447 \\
(0.5)\end{array}$ & $\begin{array}{l}15.24 \quad(50) \\
30.48 \quad(100) \\
45.72(150) \\
60.96(200)\end{array}$ & $\begin{array}{l}6000 \\
7600 \\
8000\end{array}$ & $\begin{aligned} 2.79 & (0.11) \\
5.84 & (0.23) \\
10.67 & (0.42)\end{aligned}$ & $\begin{array}{ll}1.02 & (0.04) \\
3.81 & (0.15) \\
9.40 & (0.37)\end{array}$ & $\begin{array}{l}48.6 \\
50.8 \\
22.8\end{array}$ & $\begin{array}{l}0.021 \\
0.058 \\
0.124\end{array}$ \\
\hline & $\begin{array}{r}6895 \\
(1.0)\end{array}$ & $\begin{array}{l}15.24 \quad(50) \\
30.48(100) \\
45.72(150) \\
60.96(200)\end{array}$ & $\begin{array}{l}3150 \\
4185 \\
5584 \\
6000\end{array}$ & $\begin{array}{r}4.32(0.17) \\
12.19(0.48) \\
14.99(0.59) \\
22.10(0.87)\end{array}$ & $\begin{aligned} 2.29 & (0.09) \\
10.16 & (0.40) \\
12.19 & (0.48) \\
22.10 & (0.87)\end{aligned}$ & $\begin{array}{r}44.9 \\
22.9 \\
22.1 \\
0\end{array}$ & $\begin{array}{l}0.034 \\
0.115 \\
0.188 \\
0.305\end{array}$ \\
\hline & $\begin{array}{l}13789 \\
(2.0)\end{array}$ & $\begin{array}{ll}15.24 & (50) \\
30.48 & (100) \\
45.72 & (150) \\
60.96 & (200)\end{array}$ & $\begin{array}{l}1865 \\
2879 \\
3.447 \\
* *\end{array}$ & $\begin{aligned} 7.62 & (0.30) \\
13.72 & (0.54) \\
25.40 & (1.00)\end{aligned}$ & $\begin{array}{r}5.08(0.20) \\
6.60(0.26) \\
16.00(0.63)\end{array}$ & $\begin{array}{l}33.9 \\
41.3 \\
24.4\end{array}$ & $\begin{array}{l}0.066 \\
0.171 \\
0.305\end{array}$ \\
\hline & $\begin{array}{r}3447 \\
(0.5)\end{array}$ & $\begin{array}{l}15.24 \quad(50) \\
30.48 \quad(100) \\
45.72(150) \\
60.96(200)\end{array}$ & & & & & \\
\hline & $\begin{array}{r}6895 \\
(1.0)\end{array}$ & $\begin{array}{l}15.24 \quad(50) \\
30.48(100) \\
45.72(150) \\
60.96(200)\end{array}$ & & & & & \\
\hline & $\begin{array}{l}13789 \\
(2.0)\end{array}$ & $\begin{array}{l}15.24(50) \\
30.48 \\
45.72(100) \\
60.96(150) \\
(200)\end{array}$ & & & & & \\
\hline
\end{tabular}

$*_{\mathrm{K}}=$ Energy Absorption Efficiency **Critical Strain Exceeded 
Table A-6. Impact Data for BKC 44302

\begin{tabular}{|c|c|c|c|c|c|c|c|}
\hline $\begin{array}{l}\text { Sample } \\
\text { Density } \\
\left(\mathrm{kg} / \mathrm{m}^{3}\right) \\
\left(1 \mathrm{~b} / \mathrm{ft}^{3}\right)\end{array}$ & $\begin{array}{l}\text { Impact } \\
\text { Load } \\
(\text { pa) } \\
\left(1 \mathrm{~b} / \text { in. }{ }^{2}\right)\end{array}$ & $\begin{array}{l}\text { Impact } \\
\text { Velocity } \\
(\mathrm{m} / \mathrm{s}) \\
(\mathrm{ft} / \mathrm{sec})\end{array}$ & $\begin{array}{l}\text { Maximum } \\
\text { Accel } \\
\text { (g) }\end{array}$ & $\begin{array}{l}\text { Maximum } \\
\text { Deflection } \\
(\mathrm{mm})(\text { in.) }\end{array}$ & $\begin{array}{l}\text { Final } \\
\text { Deflection } \\
(\mathrm{mm}) \text { (in.) }\end{array}$ & $\begin{array}{l}\text { Rebound } \\
\text { Velocity } \\
\text { (Percent) }\end{array}$ & $\mathrm{K}^{*}$ \\
\hline \multirow[t]{3}{*}{$\begin{array}{l}160 \\
(10)\end{array}$} & $\begin{array}{r}3447 \\
(0.5)\end{array}$ & $\begin{array}{ll}15.24 & (50) \\
30.48 & (100) \\
45.72 & (150) \\
60.96 & (200)\end{array}$ & $\begin{array}{l}1178 \\
1466 \\
2109\end{array}$ & $\begin{array}{r}9.40 \\
22.86(0.37) \\
42.67(0.90) \\
(1.68)\end{array}$ & $\begin{array}{r}7.37(0.29) \\
20.07(0.79) \\
42.67(1.68)\end{array}$ & $\begin{array}{r}30.9 \\
16.0 \\
0\end{array}$ & $\begin{array}{l}0.100 \\
0.321 \\
0.489\end{array}$ \\
\hline & $\begin{array}{r}6895 \\
(1.0)\end{array}$ & $\begin{array}{ll}15.24 & (50) \\
30.48 & (100) \\
45.72 & (150) \\
60.96 & (200)\end{array}$ & $\begin{array}{r}612 \\
1113 \\
* *\end{array}$ & $\begin{array}{l}15.49(0.61) \\
38.86(1.53)\end{array}$ & $\begin{array}{l}(0.50) \\
(1.50)\end{array}$ & $\begin{array}{r}26.2 \\
5.4\end{array}$ & $\begin{array}{l}0.185 \\
0.432\end{array}$ \\
\hline & $\begin{array}{l}13789 \\
(2.0)\end{array}$ & 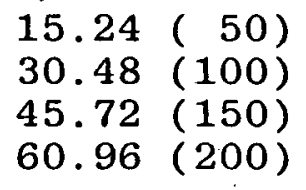 & $\begin{array}{r}352 \\
1718 \\
* *\end{array}$ & $\begin{array}{l}23.88(0.94) \\
53.09(2.09)\end{array}$ & $\begin{array}{l}20.07 \quad(0.79) \\
53.09 \quad(2.09)\end{array}$ & $\begin{array}{r}19.2 \\
0\end{array}$ & $\begin{array}{l}0.310 \\
0.273\end{array}$ \\
\hline \multirow[t]{3}{*}{$\begin{array}{l}320 \\
(20)\end{array}$} & $\begin{array}{r}3447 \\
(0.5)\end{array}$ & $\begin{array}{l}15.24 \quad(50) \\
30.48(100) \\
45.72(150) \\
60.96(200)\end{array}$ & $\begin{array}{l}3900 \\
4083 \\
4605 \\
5808\end{array}$ & $\begin{array}{r}4.32(0.17) \\
11.43(0.45) \\
18.03(0.71) \\
23.88(0.94)\end{array}$ & $\begin{array}{r}2.29(0.09) \\
9.40(0.37) \\
17.27(0.68) \\
20.57(0.81)\end{array}$ & $\begin{array}{r}51.3 \\
24.4 \\
4.5 \\
14.0\end{array}$ & $\begin{array}{l}0.031 \\
0.115 \\
0.220 \\
0.316\end{array}$ \\
\hline & $\begin{array}{r}6895 \\
(1.0)\end{array}$ & 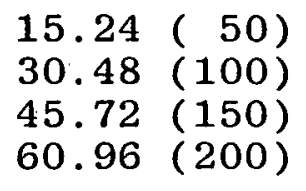 & $\begin{array}{l}2010 \\
2655 \\
3038 \\
5705\end{array}$ & 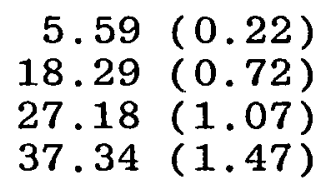 & 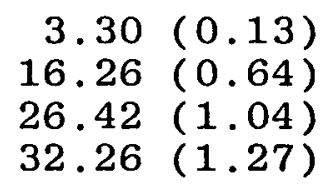 & $\begin{array}{r}39.5 \\
15.3 \\
0 \\
4.18\end{array}$ & $\begin{array}{l}0.056 \\
0.190 \\
0.339 \\
0.321\end{array}$ \\
\hline & $\begin{array}{l}13789 \\
(2.0)\end{array}$ & $\begin{array}{l}15.24 \quad(50) \\
30.48 \quad(100) \\
45.72(150) \\
60.96(200)\end{array}$ & $\begin{array}{l}1033 \\
1688 \\
* *\end{array}$ & $\begin{array}{l}11.43(0.45) \\
23.88(0.94)\end{array}$ & $\begin{array}{r}9.65(0.38) \\
20.32(0.80)\end{array}$ & $\begin{array}{l}21.8 \\
18.1\end{array}$ & $\begin{array}{l}0.113 \\
0.271\end{array}$ \\
\hline
\end{tabular}


Table A-6 Continued. Impact Data for BKC 44302

\begin{tabular}{|c|c|c|c|c|c|c|c|}
\hline $\begin{array}{l}\text { Sample } \\
\text { Density } \\
\left(\mathrm{kg} / \mathrm{m}^{3}\right) \\
\left(1 \mathrm{~b} / \mathrm{ft}^{3}\right)\end{array}$ & $\begin{array}{l}\text { Impact } \\
\text { Load } \\
(\text { pa) } \\
(1 \mathrm{~b} / \text { in } .2)\end{array}$ & $\begin{array}{l}\text { Impact } \\
\text { Velocity } \\
(\mathrm{m} / \mathrm{s}) \\
(\mathrm{ft} / \mathrm{sec})\end{array}$ & $\begin{array}{l}\text { Maximum } \\
\text { Accel } \\
(\mathrm{g})\end{array}$ & $\begin{array}{l}\text { Maximum } \\
\text { Deflection } \\
(\mathrm{mm}) \text { (in.) }\end{array}$ & $\begin{array}{l}\text { Final } \\
\text { Deflection } \\
(\mathrm{mm}) \text { (in.) }\end{array}$ & $\begin{array}{l}\text { Rebound } \\
\text { Velocity } \\
\text { (Percent) }\end{array}$ & $\mathrm{K}^{*}$ \\
\hline \multirow[t]{4}{*}{$\begin{array}{l}480 \\
(30)\end{array}$} & $\begin{array}{r}3447 \\
(0.5)\end{array}$ & $\begin{array}{l}15.24 \quad(50) \\
30.48(100) \\
45.72(150) \\
60.96(200)\end{array}$ & $\begin{array}{r}5310 \\
10730 \\
10800\end{array}$ & $\begin{array}{ll}3.30 & (0.13) \\
5.08 & (0.20) \\
9.91 & (0.39)\end{array}$ & $\begin{array}{ll}2.29 & (0.09) \\
2.29 & (0.09) \\
8.64 & (0.34)\end{array}$ & $\begin{array}{l}34.0 \\
57.1 \\
19.4\end{array}$ & $\begin{array}{l}0.022 \\
0.047 \\
0.095\end{array}$ \\
\hline & $\begin{array}{l}13789 \\
(2.0)\end{array}$ & $\begin{array}{l}15.24 \quad(50) \\
30.48 \quad(100) \\
45.72 \cdot(150) \\
60.96(200)\end{array}$ & $\begin{array}{l}2624 \\
3527 \\
5578\end{array}$ & $\begin{array}{r}3.56(0.14) \\
12.45(0.49) \\
29.72(1.17)\end{array}$ & $\begin{array}{r}0.76(0.03) \\
8.38(0.33) \\
28.19(1.11)\end{array}$ & $\begin{array}{l}59.8 \\
38.2 \\
10.6\end{array}$ & $\begin{array}{l}0.041 \\
0.135 \\
0.329\end{array}$ \\
\hline & $\begin{array}{r}6895 \\
(1.0)\end{array}$ & $\begin{array}{l}15.24 \quad(50) \\
30.48 \quad(100) \\
45.72(150) \\
60.96(200)\end{array}$ & & & & & \\
\hline & $\begin{array}{l}13789 \\
(2.0)\end{array}$ & $\begin{array}{ll}15.24 & (50) \\
30.48 & (100) \\
45.72 & (150) \\
60.96 & (200)\end{array}$ & & & & & \\
\hline
\end{tabular}




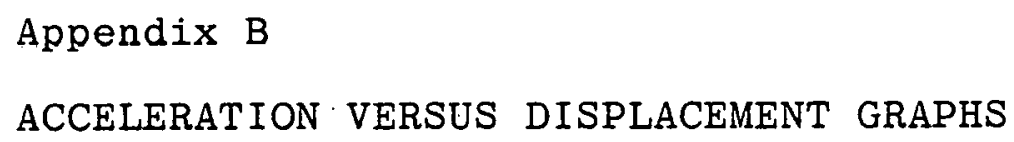


ACCELERATION VS DISPLACEMENT
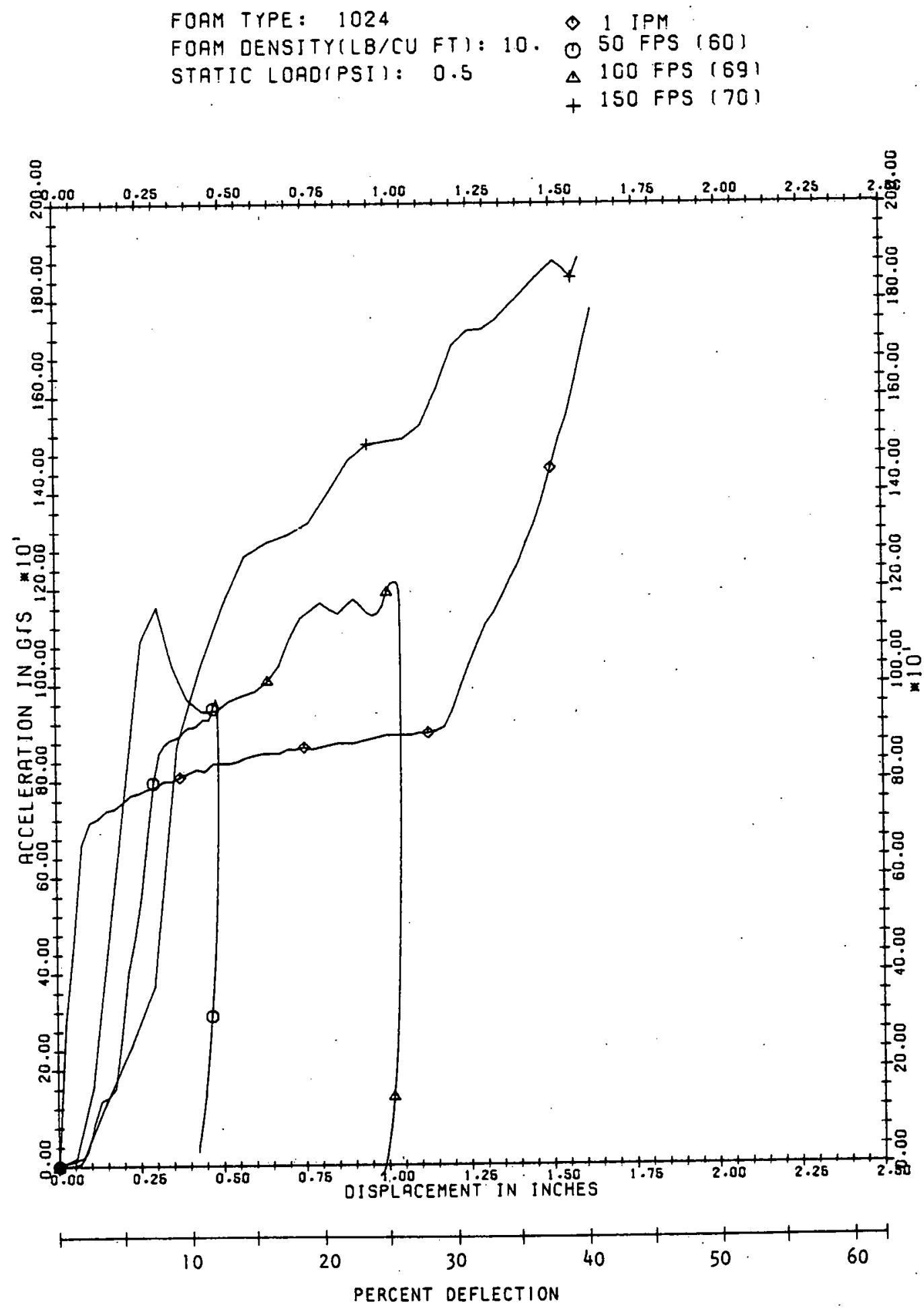

Figure B-1. CPR 1024, $10 \mathrm{LB} / \mathrm{FT}^{3}, 0.5 \mathrm{PSI}$ 
ACCELERATION VS OISPLACEMENT
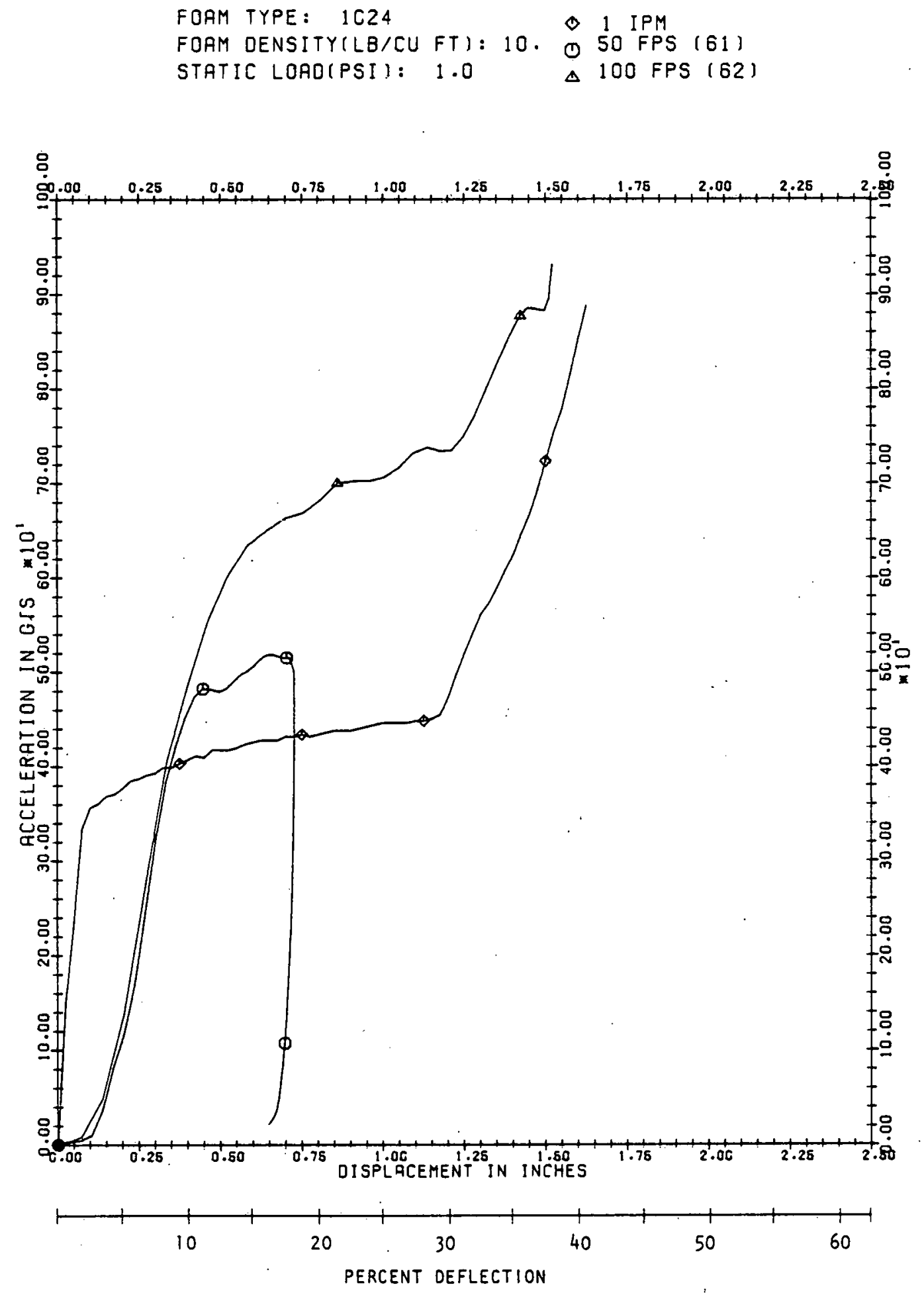

Figure B-2. CPR 1024, $10 \mathrm{LB} / \mathrm{FT}^{3}, 1.0 \mathrm{PSI}$ 

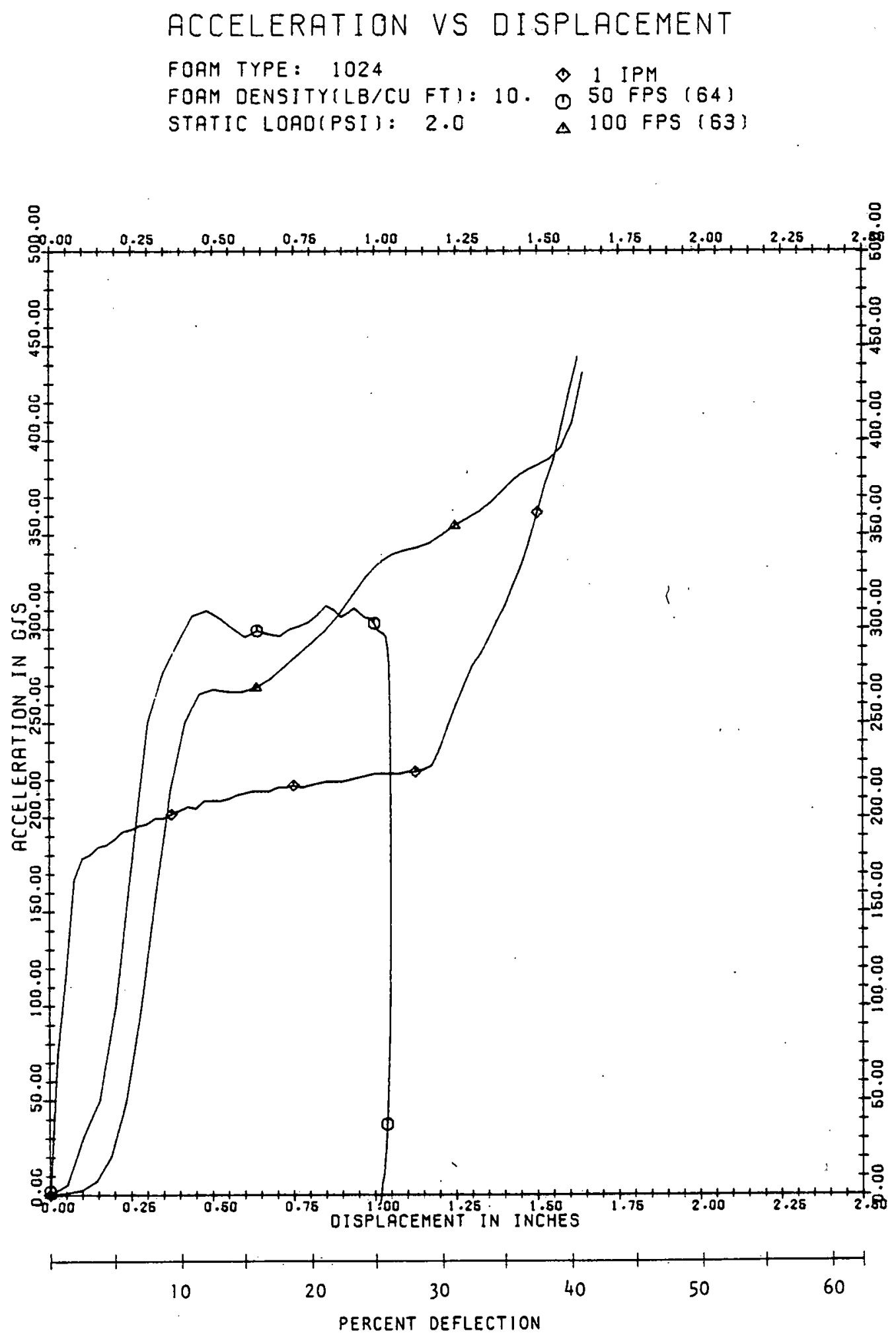

Figure B-3. CPR 1024, $10 \mathrm{LB} / \mathrm{FT}^{3}, 2.0 \mathrm{PSI}$ 

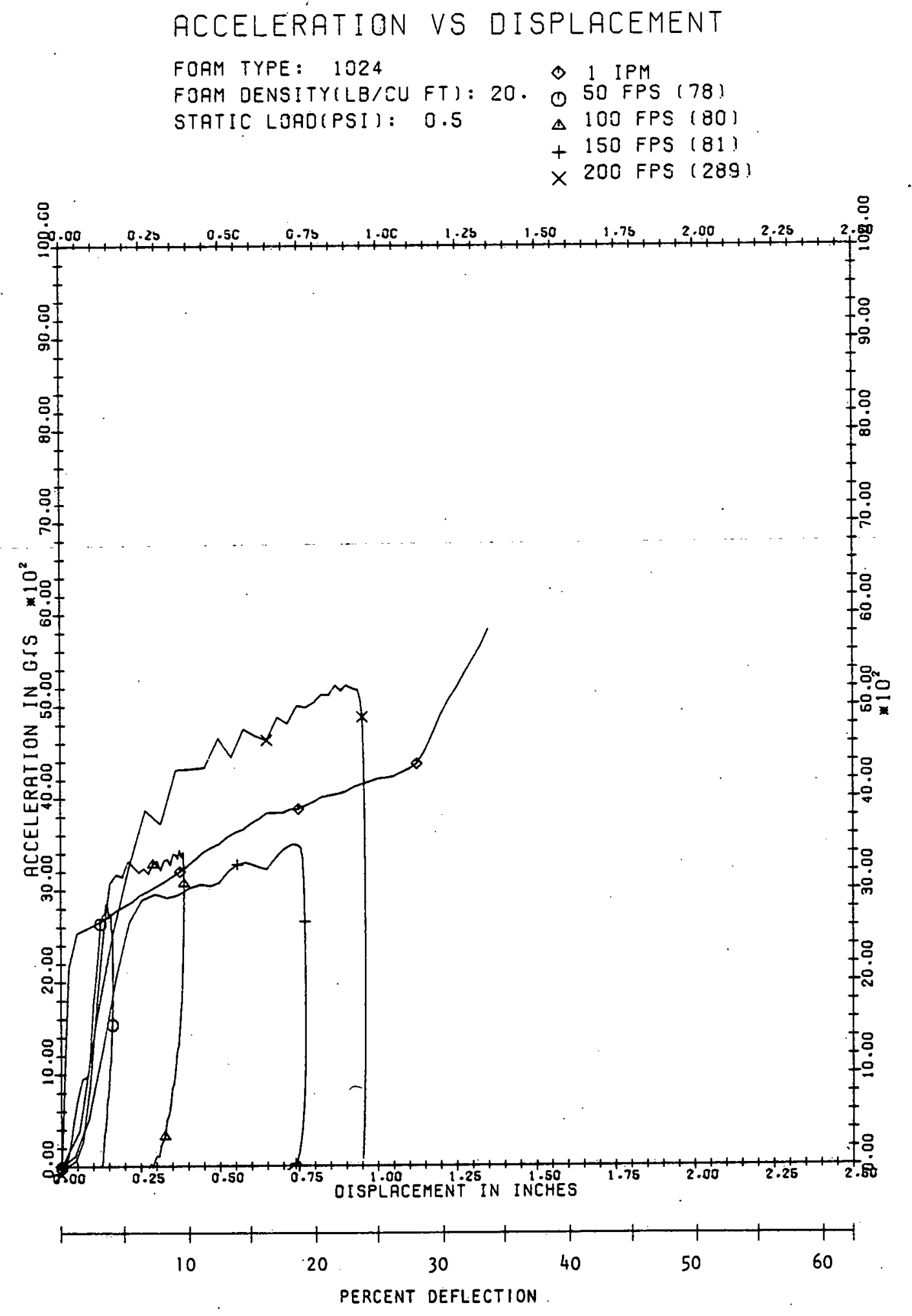

Figure B-4. CPR 1024, $20 \mathrm{LB} / \mathrm{FT}^{3}, 0.5 \mathrm{PSI}$ 


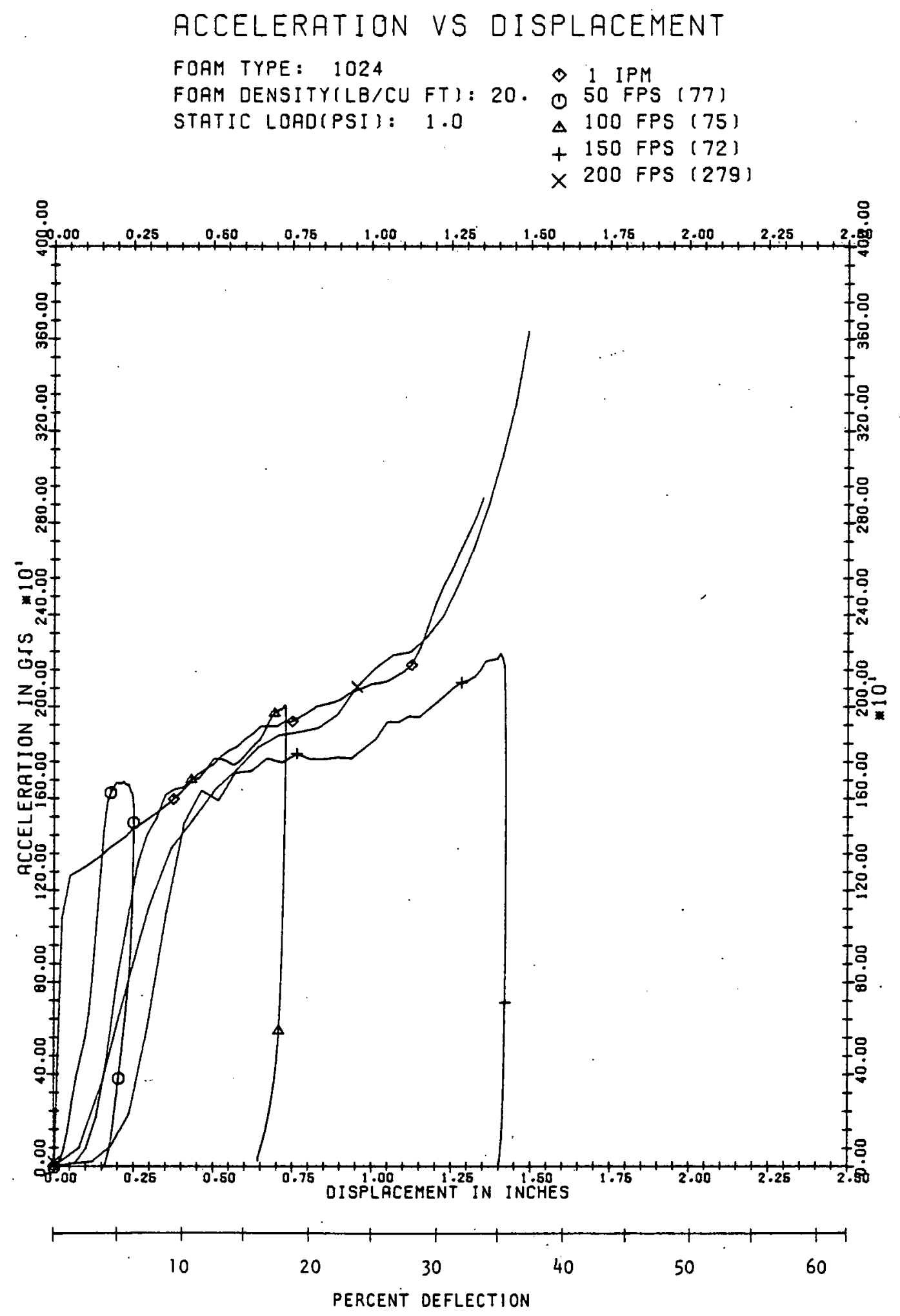

Figure B-5. CPR 1024, $20 \mathrm{LB} / \mathrm{FT}^{3}, 1.0 \mathrm{PSI}$ 

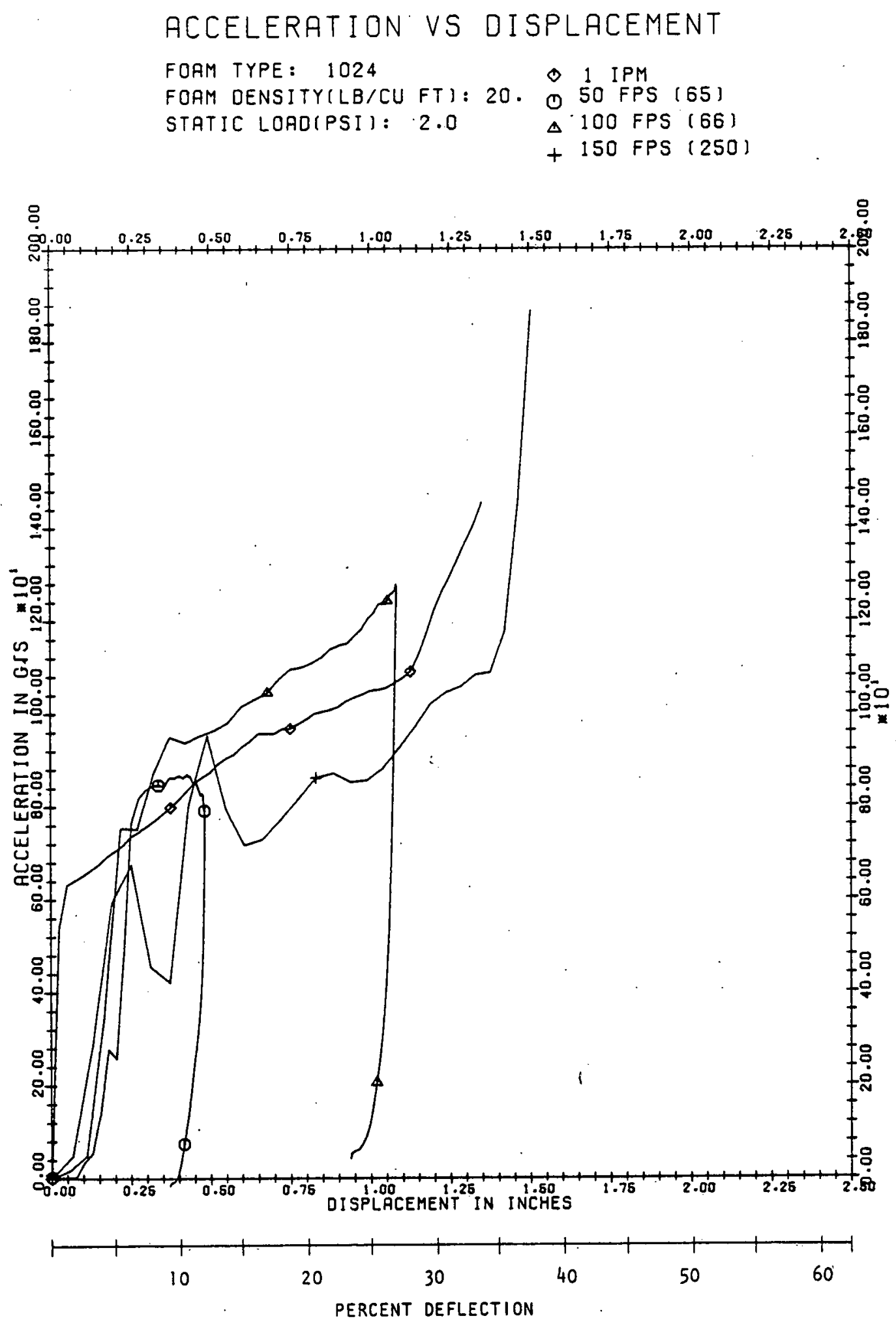

Figure B-6. CPR 1024, $20 \mathrm{LB} / \mathrm{FT}^{3}, 2.0 \mathrm{PSI}$ 
ACCELERATION VS DISPLACEMENT
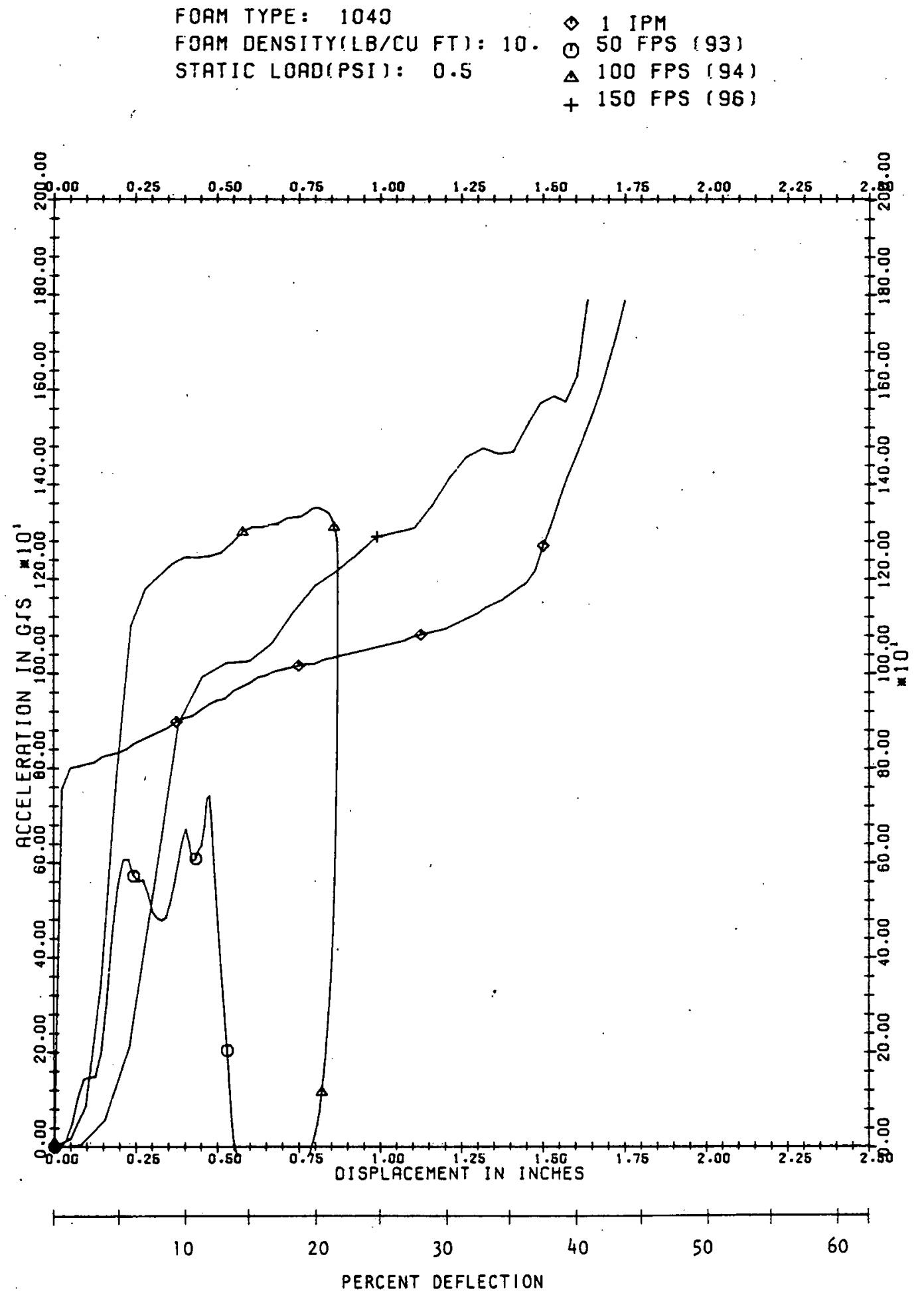

Figure B-7. CPR 1040, $10 \mathrm{LB}^{-\mathrm{FT}^{3}}, 0.5 \mathrm{PSI}$ 
ACCELERATION VS DISPLACEMENT
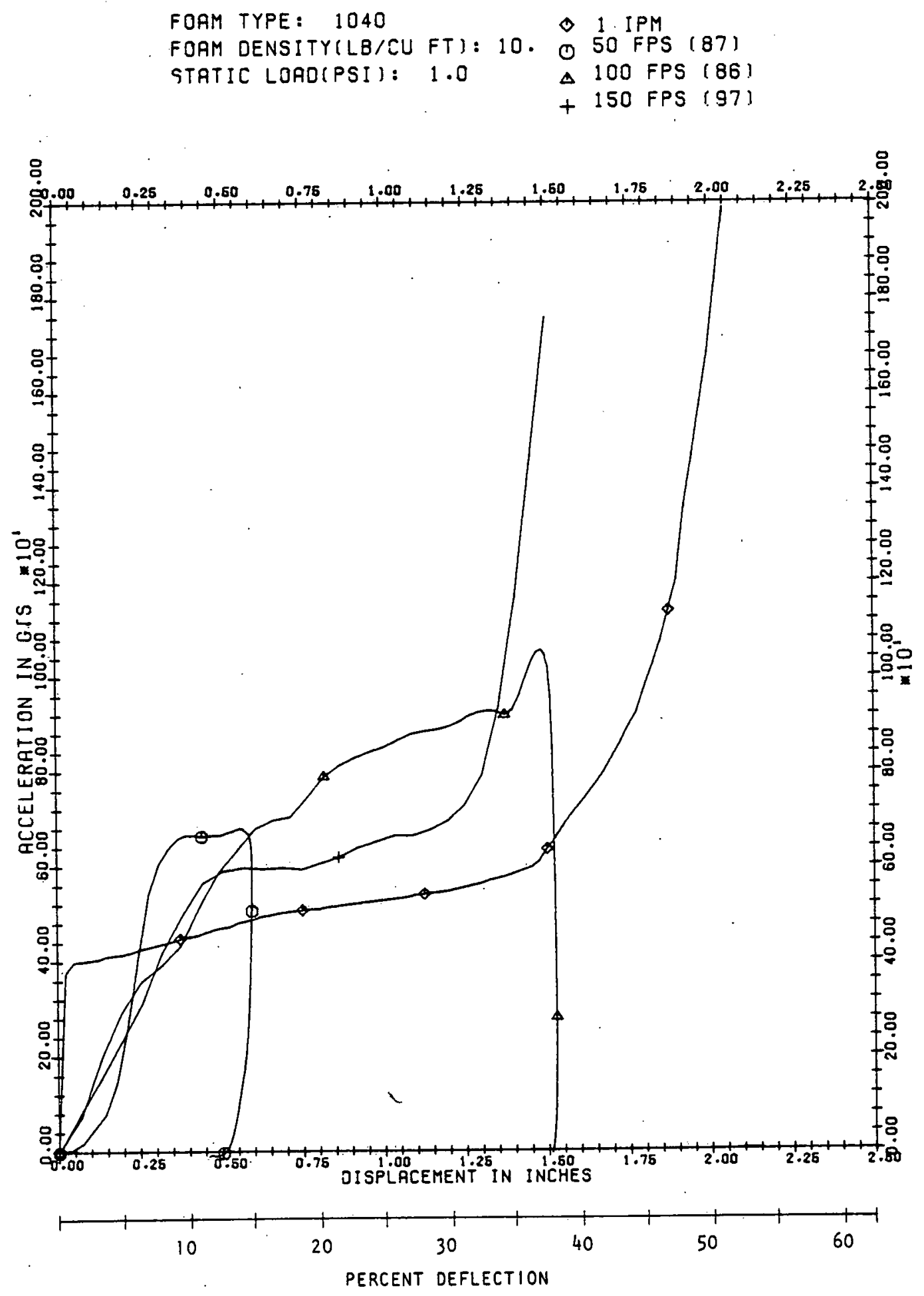

Figure B-8. CPR 1040, $10 \mathrm{LB} / \mathrm{FT}^{3}, 1.0 \mathrm{PSI}$ 
ACCELERATION VS DISPLACEMENT
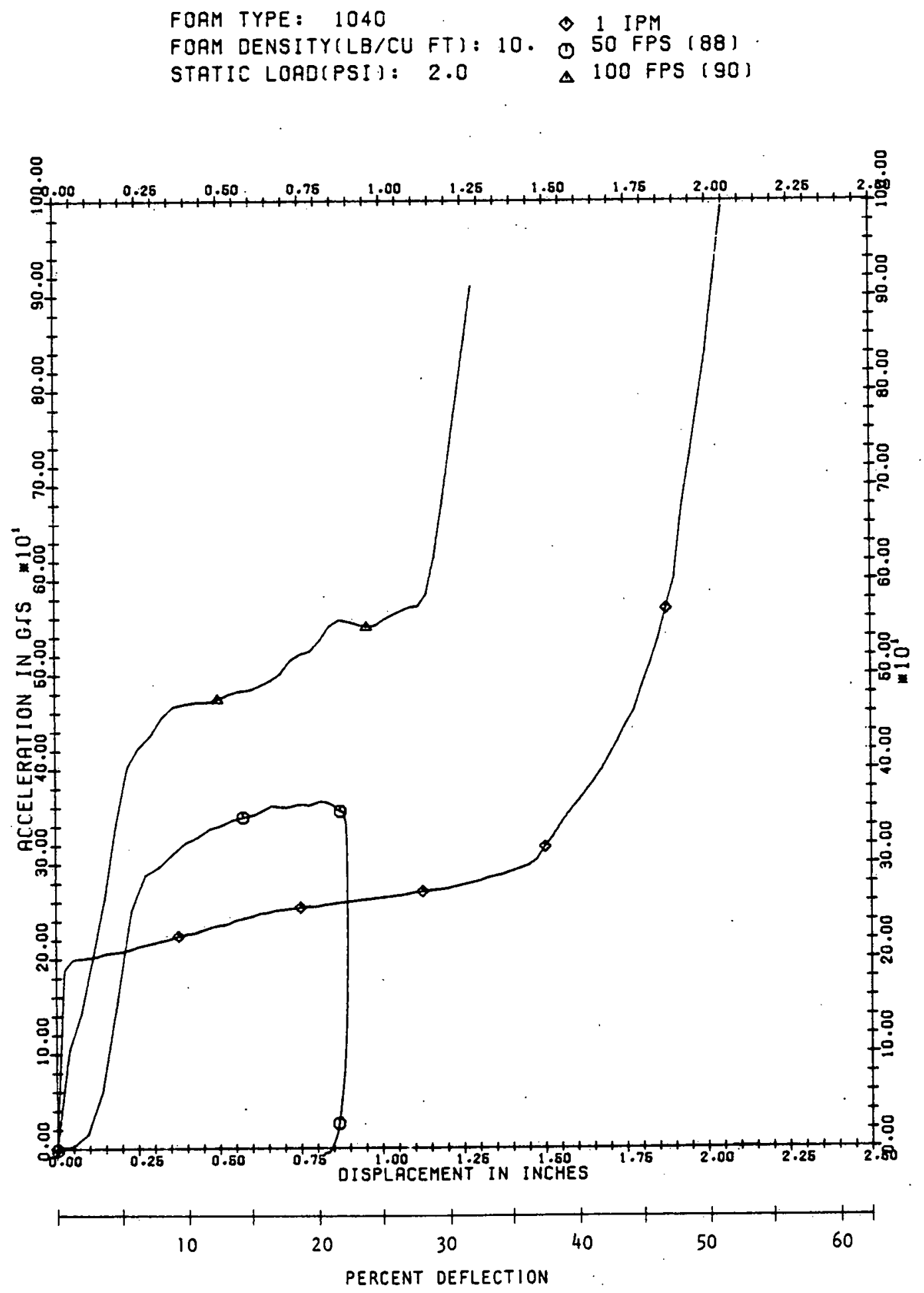

Figure B-9. CPR 1040, $10 \mathrm{LB} / \mathrm{FT}^{3}, 2.0 \mathrm{PSI}$ 
ACCELERATION VS DISPLACEMENT
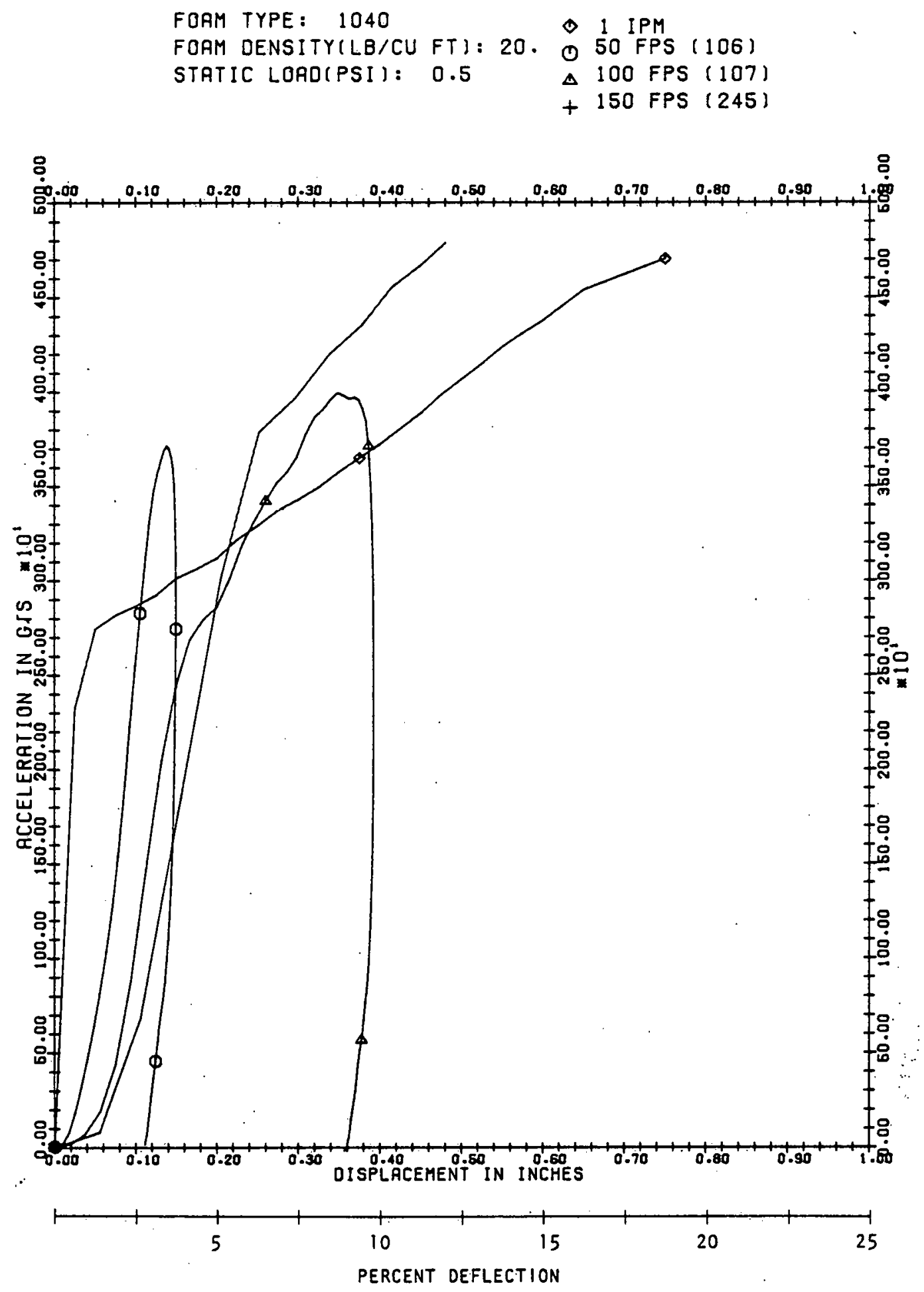

Figure $\mathrm{B}-10 . \quad \mathrm{CPR} 1040,20 \mathrm{LB} / \mathrm{FT}^{3}, 0.5 \mathrm{PSI}$ 
ACCELERATION VS DISPLACEMENT
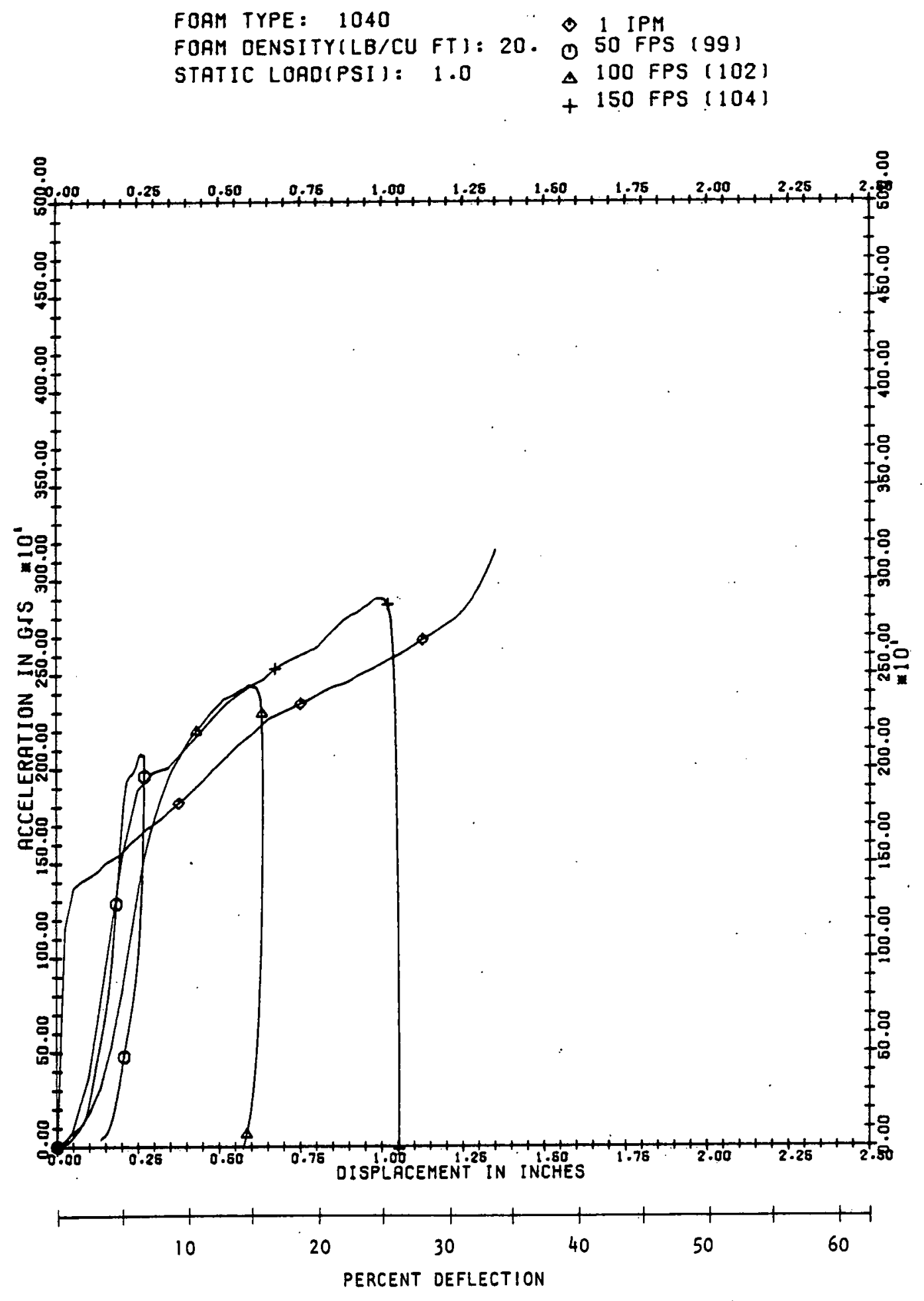

Figure B-11. CPR 1040, $20 \mathrm{LB} / \mathrm{FT}^{3}, 1.0 \mathrm{PSI}$ 

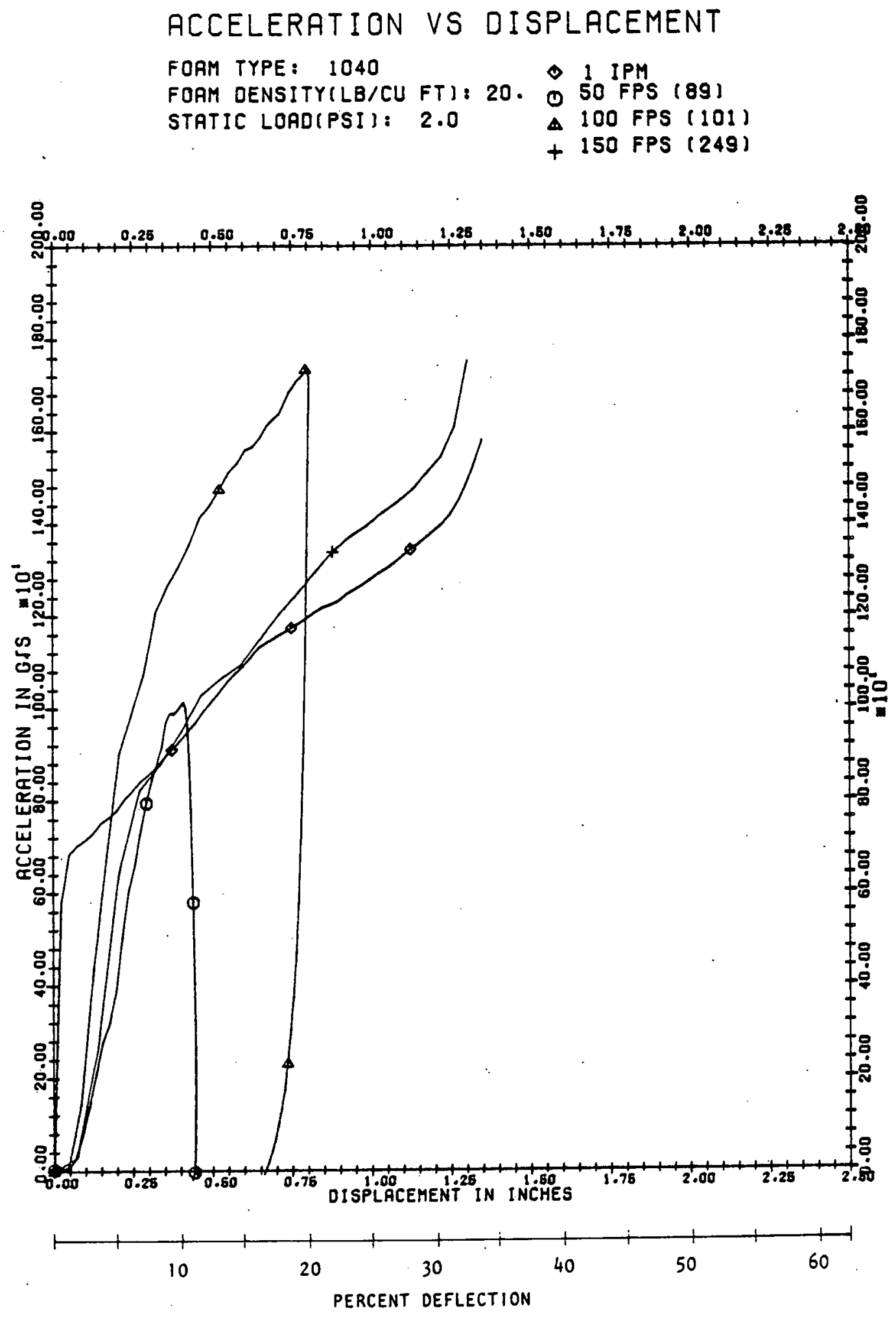

Figure B-12. CPR 1040, $20 \mathrm{LB} / \mathrm{FT}^{3}, 2.0 \mathrm{PSI}$ 
ACCELERATION VS DISPLACEMENT

FOAM TYPE: 1200

- 1 IPM

FOAM OENSITYILB/CU FTI: 10 . O 50 FPS (131)

STATIC LOAQ(PSI), 0.5

$\triangle 100$ FPS (232)

+150 FPS (129)

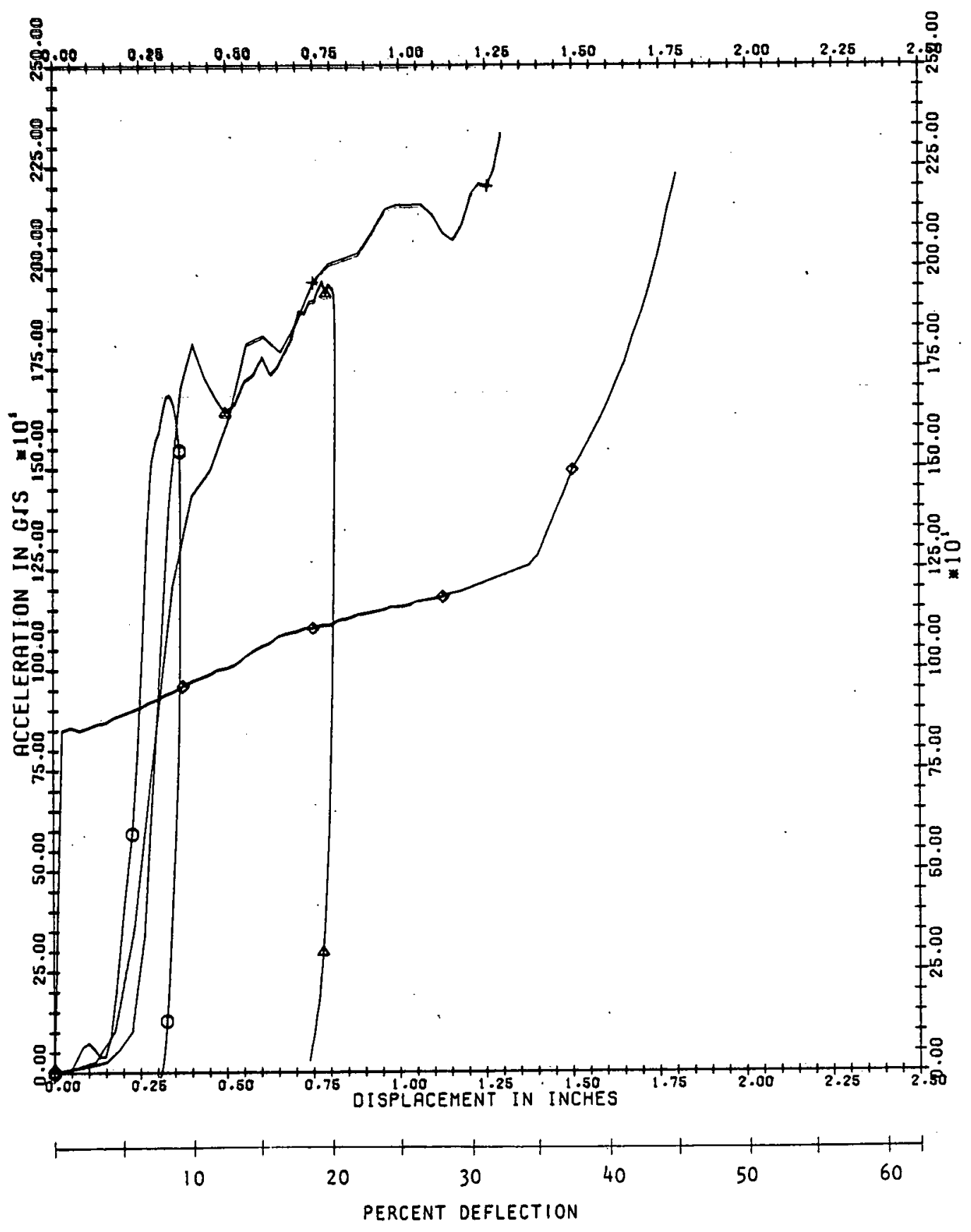

Figure B-13. $\quad \mathrm{BC} 1200,10 \mathrm{LB} / \mathrm{FT}^{3}, 0.5 \mathrm{PSI}$ 

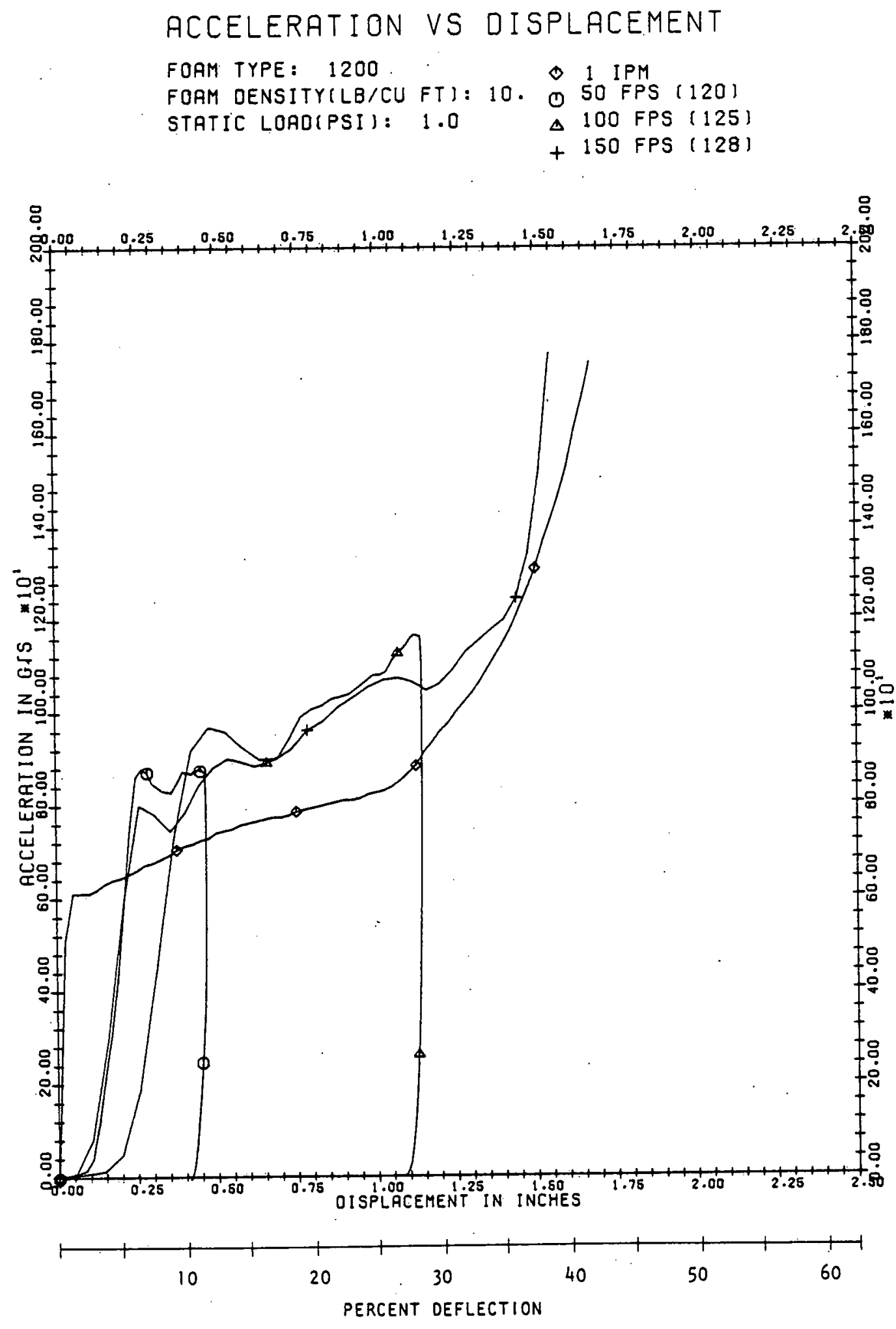

Figure B-14. $\mathrm{BC} 1200,10 \mathrm{LB} / \mathrm{FT}^{3}, 1.0 \mathrm{PSI}$ 


\section{ACCELERATION VS DISPLACEMENT}

FOAM TYPE: 1200

FOAM DENSITY(LB/CU FT): 10 .

- 1 IPM

STATIC LOROIPSII: 2.0

O 50 FPS (117)

$\triangle 100$ FPS (119)

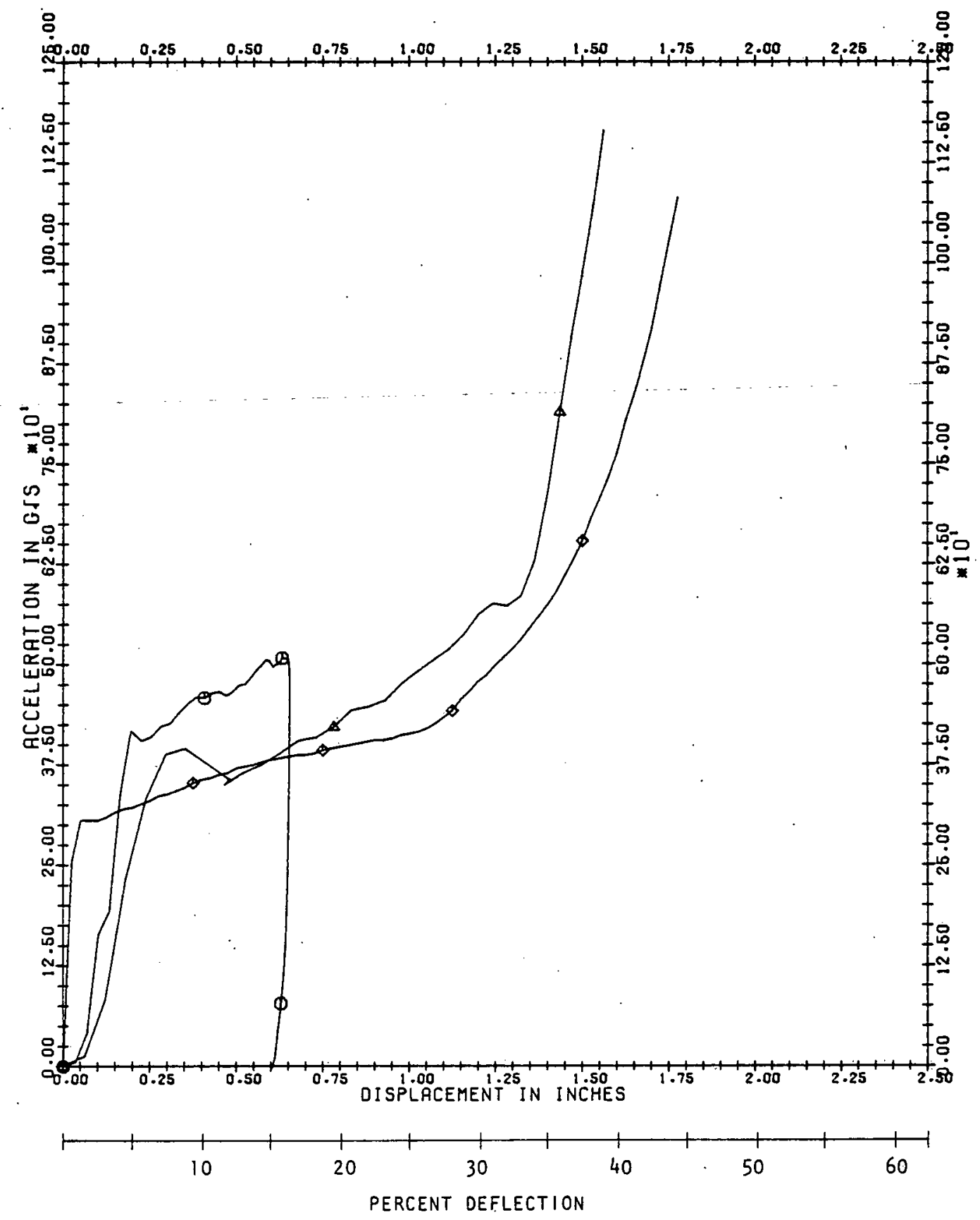

Figure B-15. BC $1200,10 \mathrm{LB} / \mathrm{FT}^{3}, 2.0 \mathrm{PSI}$ 
ACCELERATION VS DISPLACEMENT

FOAM TYPE: 1200

FORM DENSITY(LB/CU FTI: 20 .

STATIC LOAOIPSI):

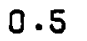

- 1 IPM

(1) 50 FPS (147)

$\triangle 100$ FPS (148)

+150 FPS $(151)$

$\times 200$ FPS (287)

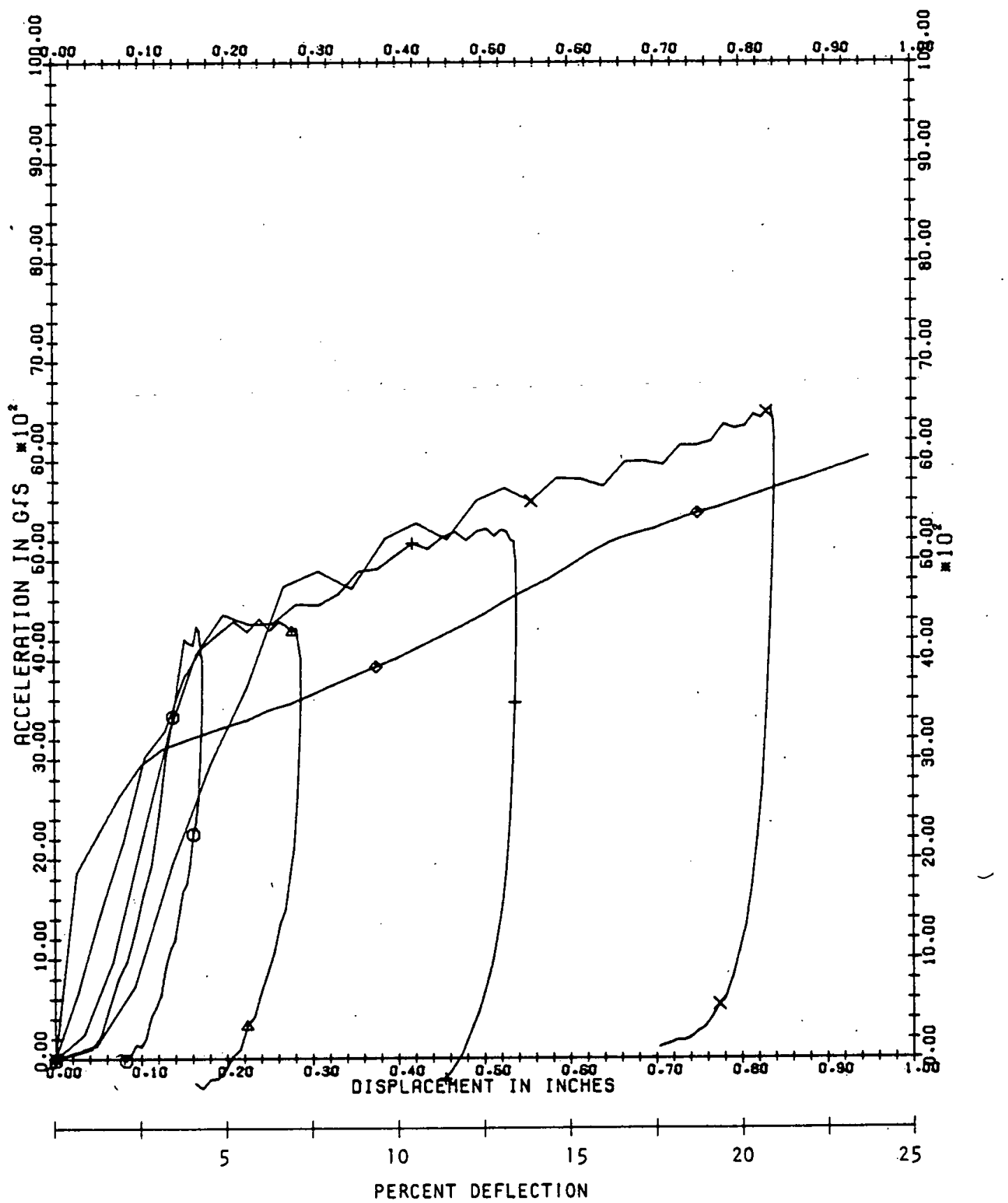

Figure B-16. BC $1200,20 \mathrm{LB} / \mathrm{FT}^{3}, 0.5 \mathrm{PSI}$ 
ACCELERATION VS DISPLACEMENT
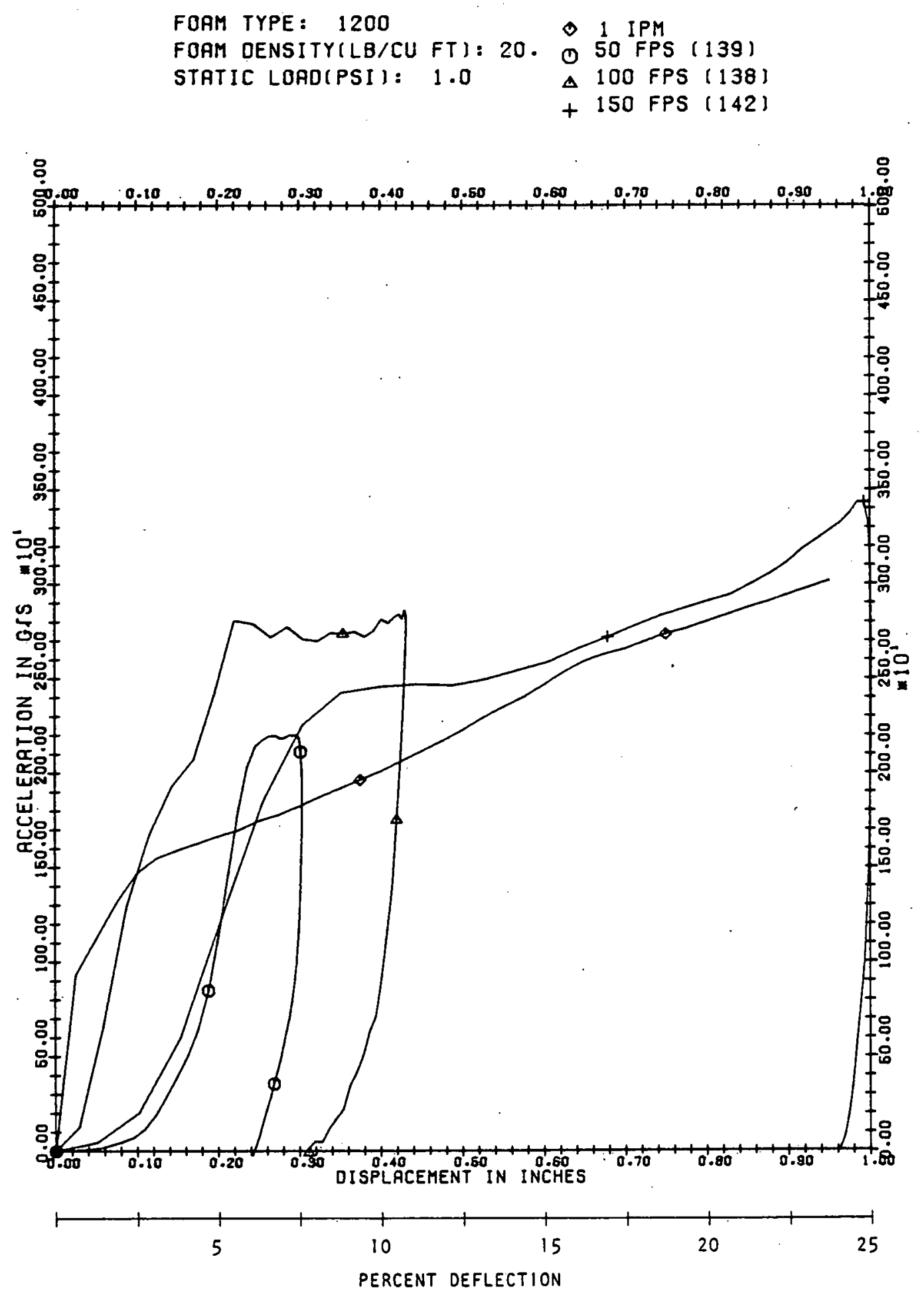

Figure B-17. BC $1200,20 \mathrm{LB} / \mathrm{FT}^{3}, 1.0 \mathrm{PSI}$ 
ACCELERATION VS DISPLACEMENT
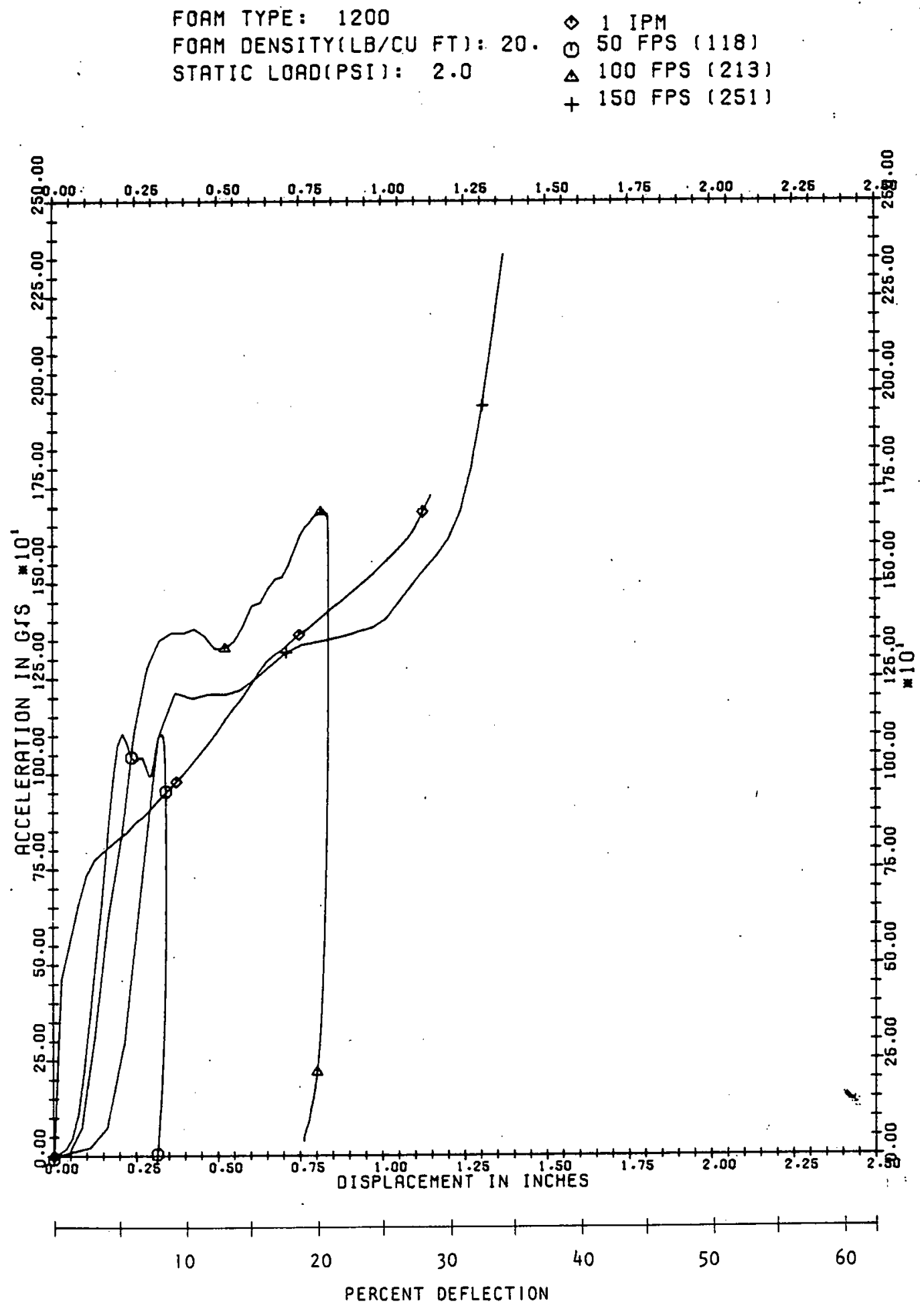

Figure B-18. BC $1200,20 \mathrm{LB} / \mathrm{FT}^{3}, 2.0 \mathrm{PSI}$ 


\section{ACCELERATION VS DISPLACEMENT}
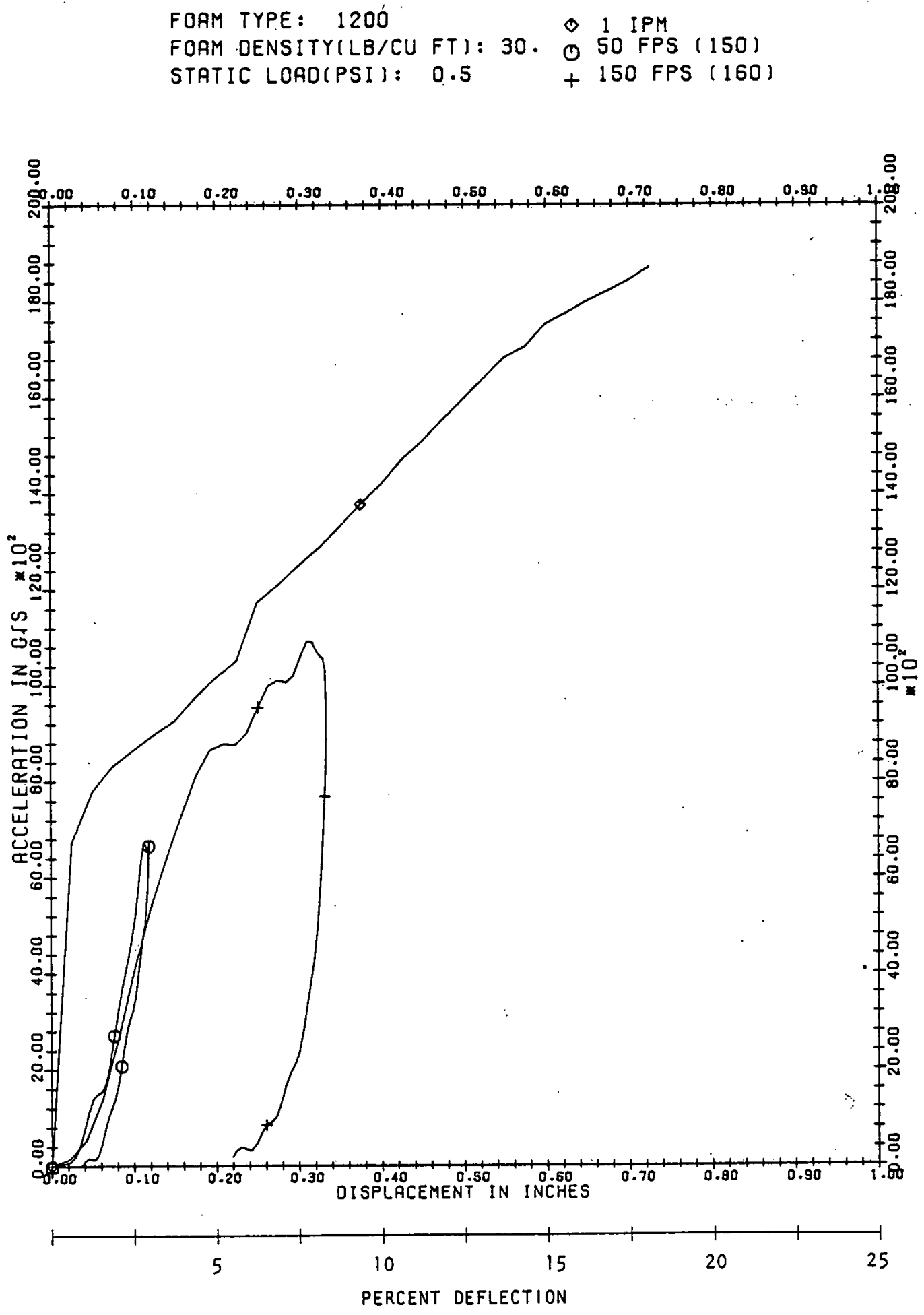

Figure B-19. $\quad \mathrm{BC} 1200,30 \mathrm{LB} / \mathrm{FT}^{3}, 0.5 \mathrm{PSI}$ 

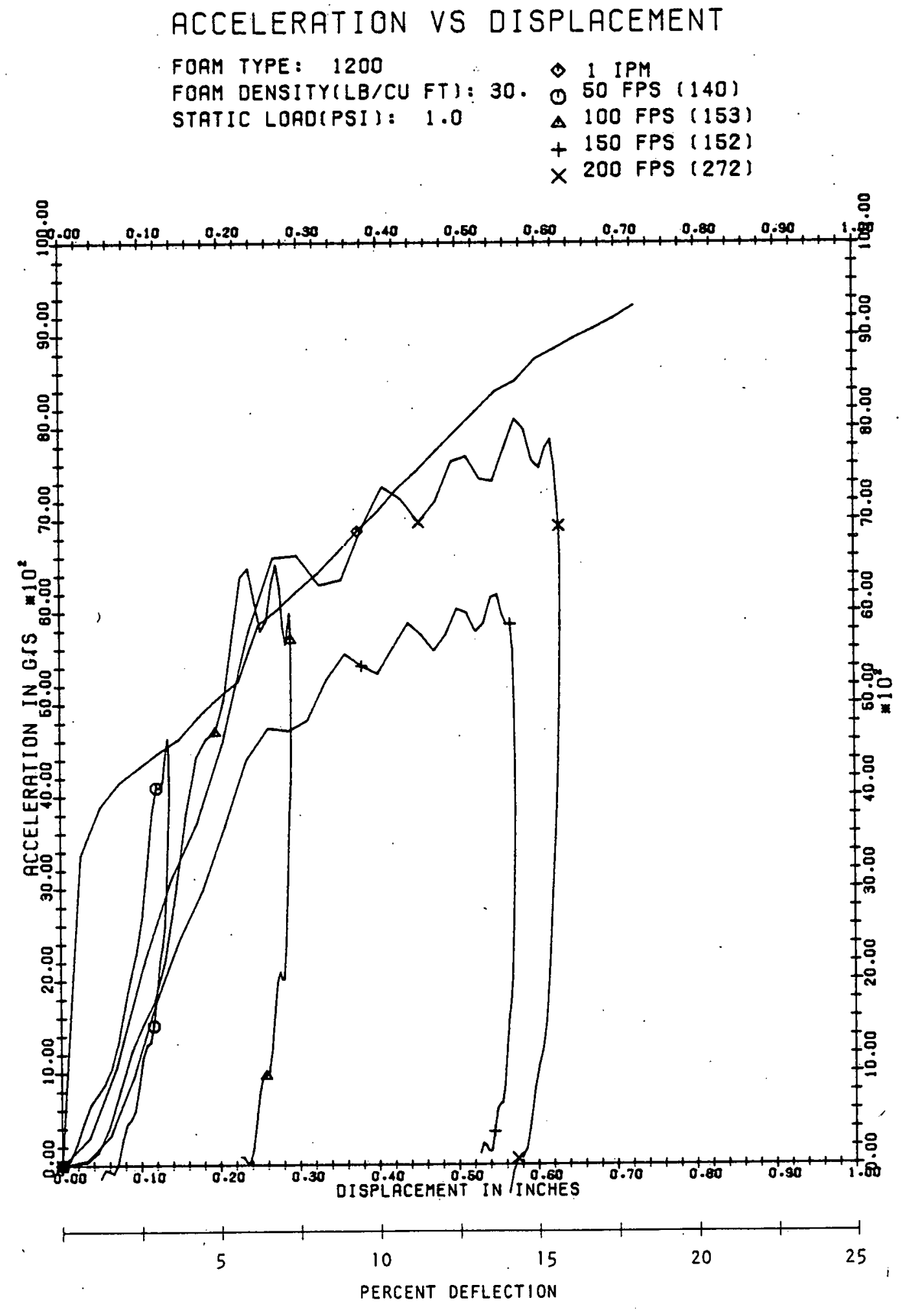

Figure $\mathrm{B}-20 . \quad \mathrm{BC} 1200,30 \mathrm{LB}^{-\mathrm{FT}^{3}}, 1.0 \mathrm{PSI}$ 

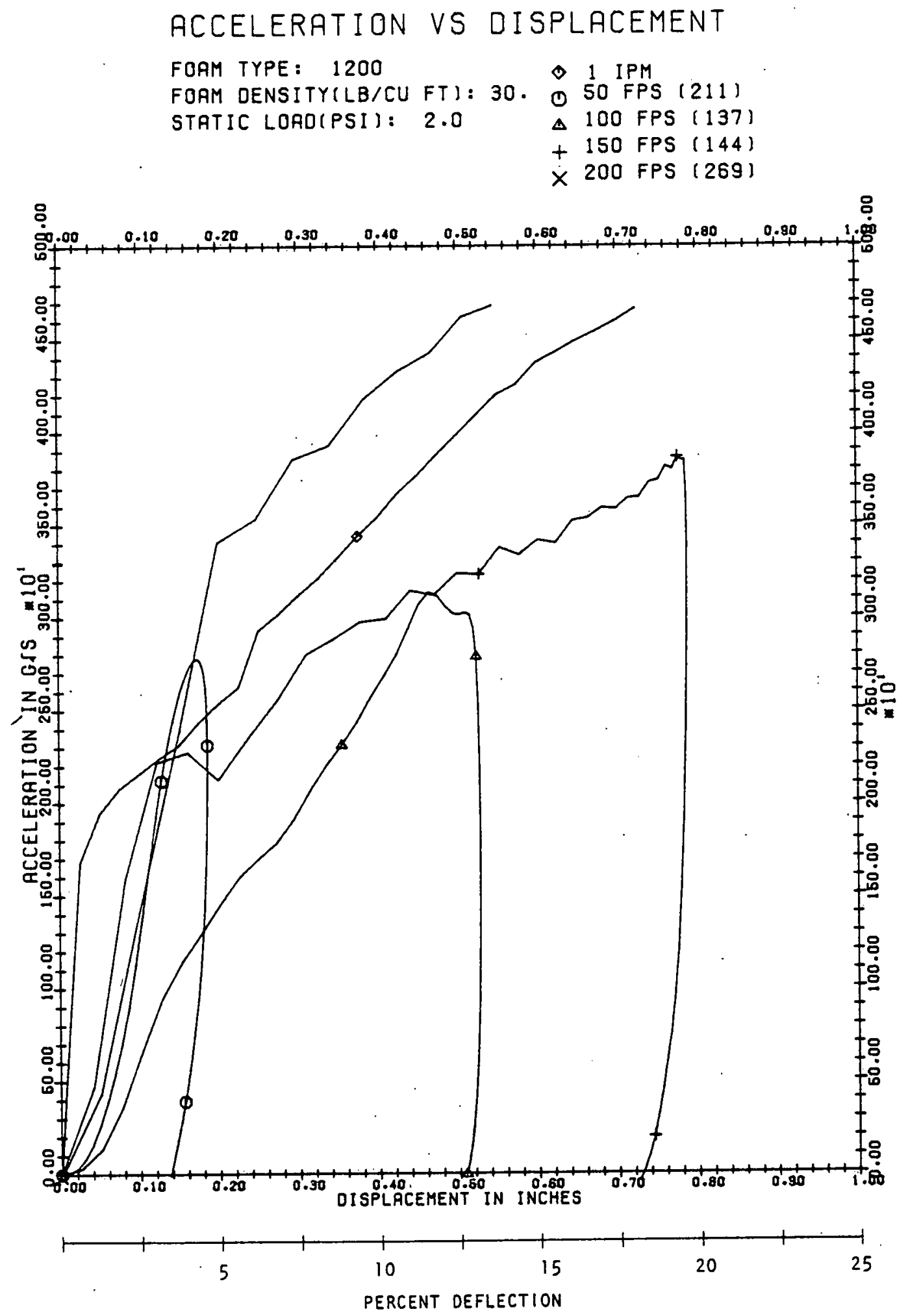

Figure B-21. BC $1200,30 \mathrm{LB} / \mathrm{FT}^{3}, 2.0 \mathrm{PSI}$ 

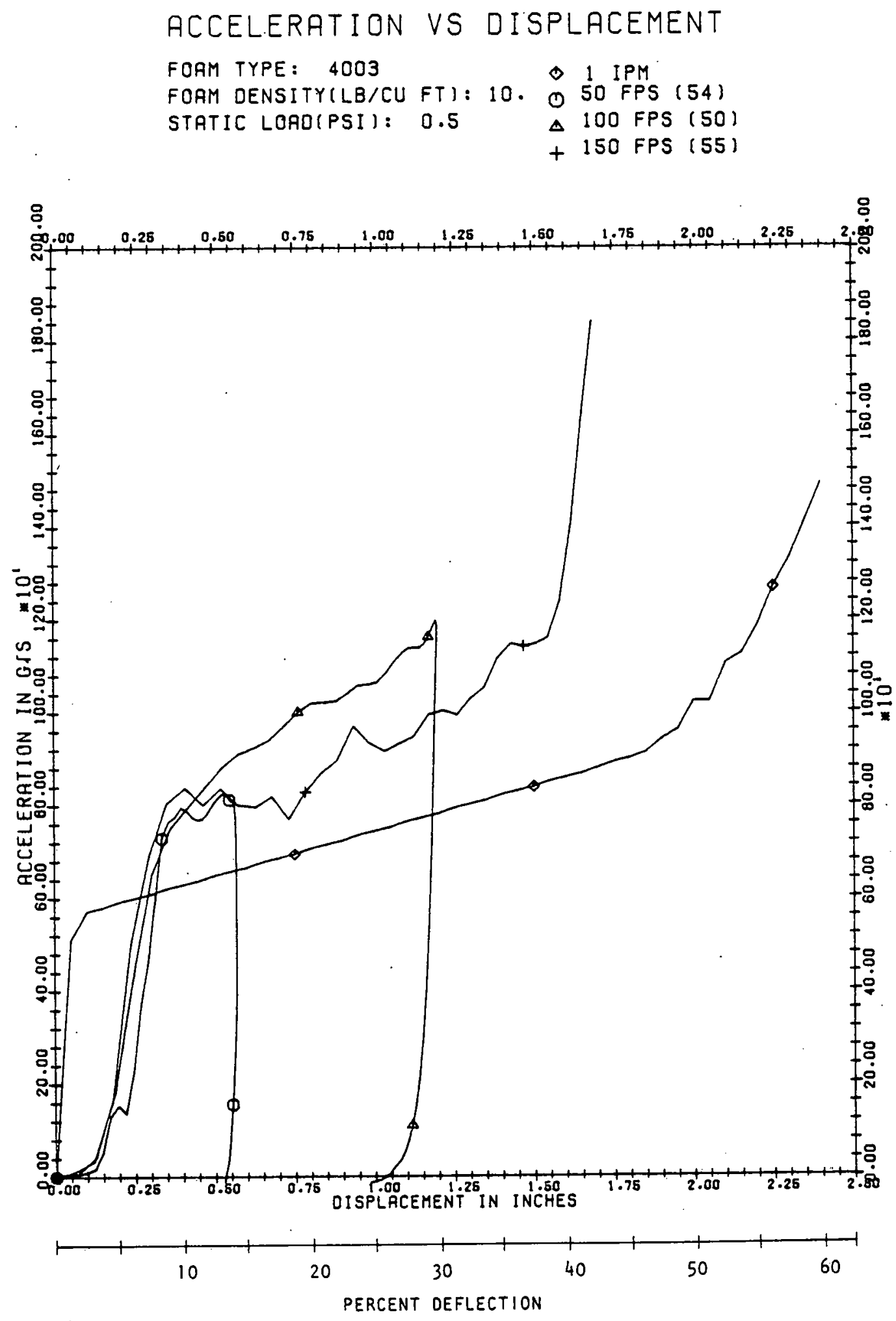

Figure B-22. $\mathrm{BX} 4003,10 \mathrm{LB} / \mathrm{FT}^{3}, 0.5 \mathrm{PSI}$ 
ACCELERATION VS DISPLACEMENT
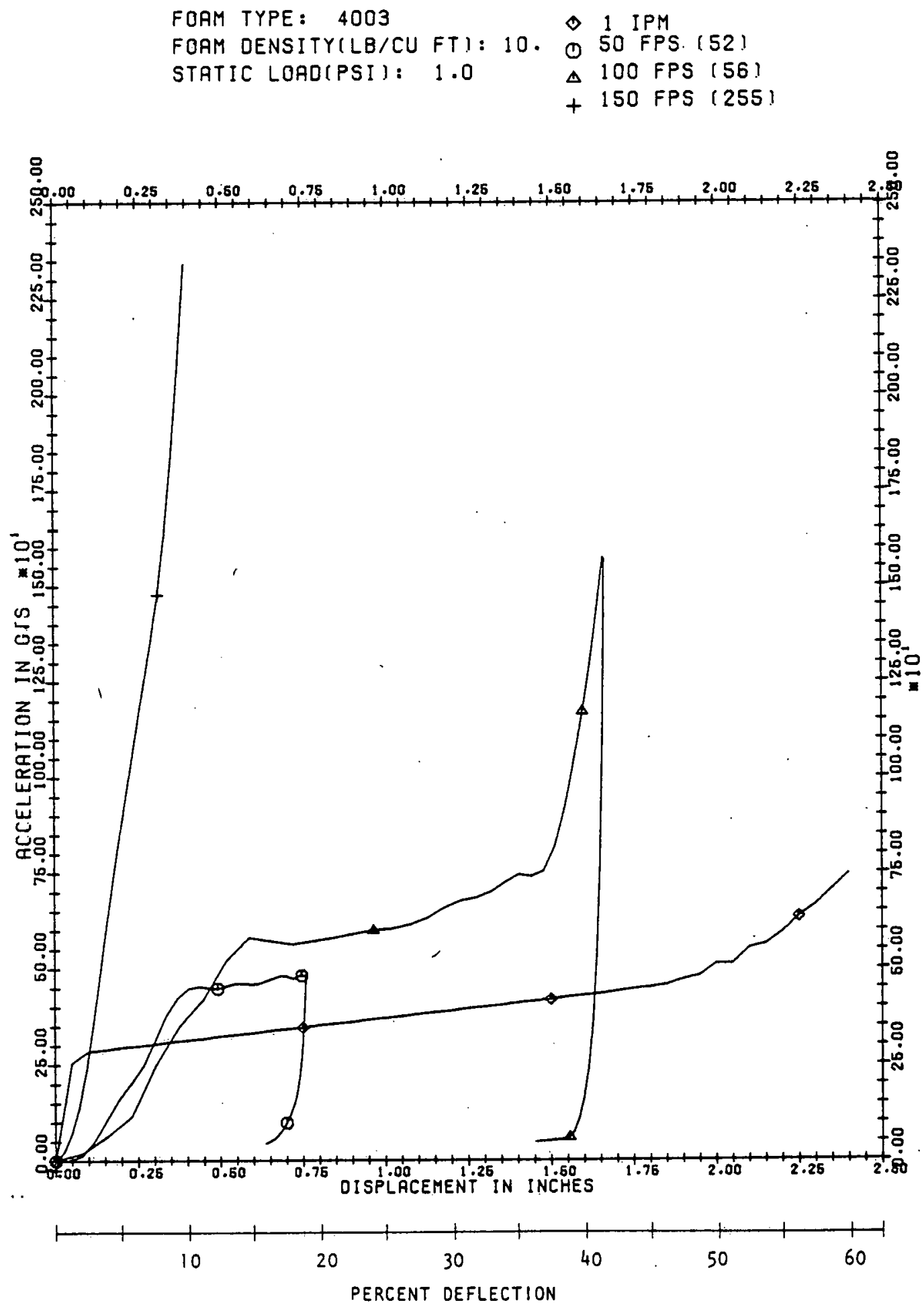

Figure B-23. $\mathrm{BX} 4003,10 \mathrm{LB} / \mathrm{FT}^{3}, 1.0 \mathrm{PSI}$ 


\section{ACCELERATION VS DISPLACEMENT}
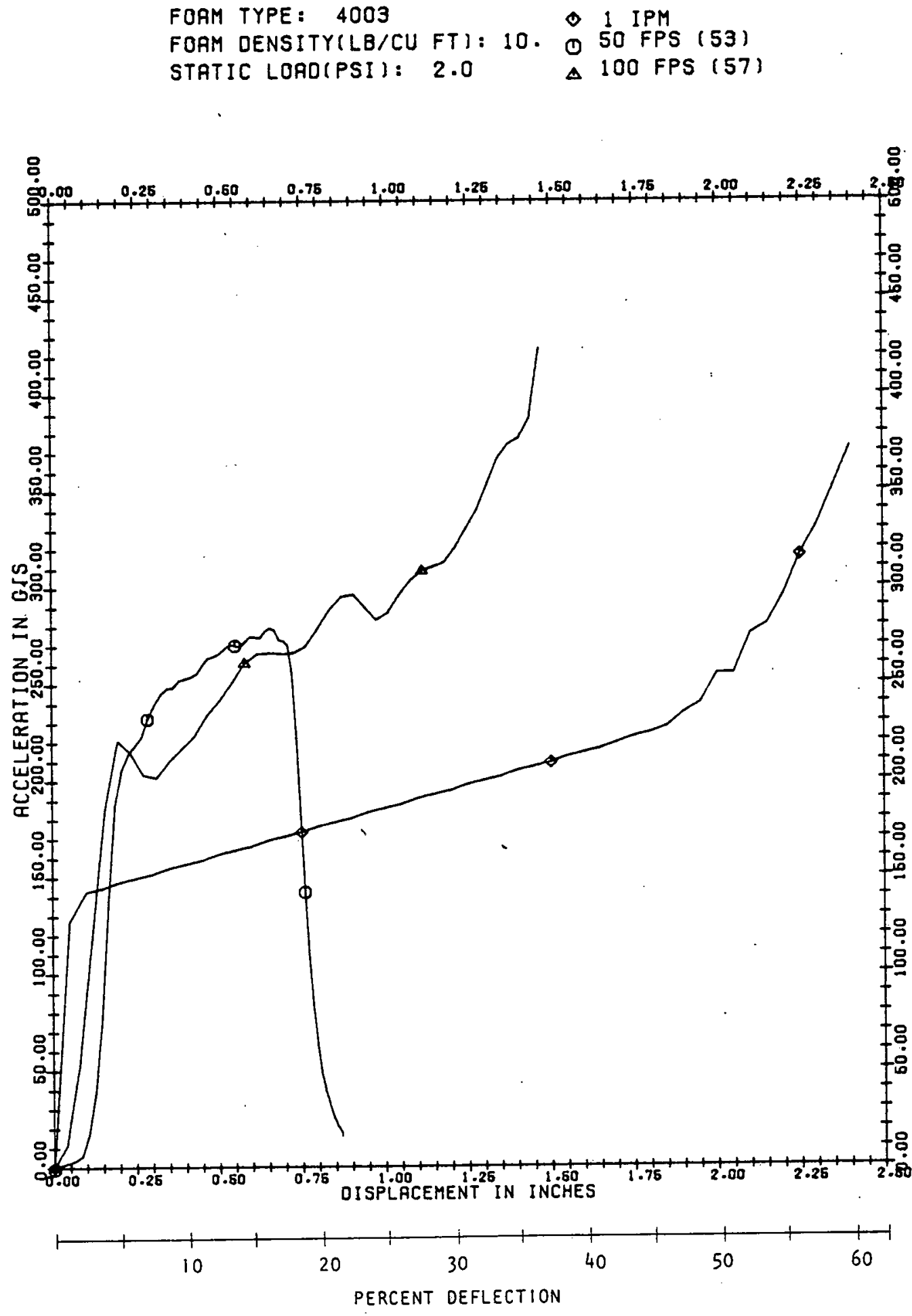

Figure B-24. BX 4003, $10 \mathrm{LB} / \mathrm{FT}^{3}, 2.0 \mathrm{PSI}$ 
ACCELERATION VS DISPLACEMENT
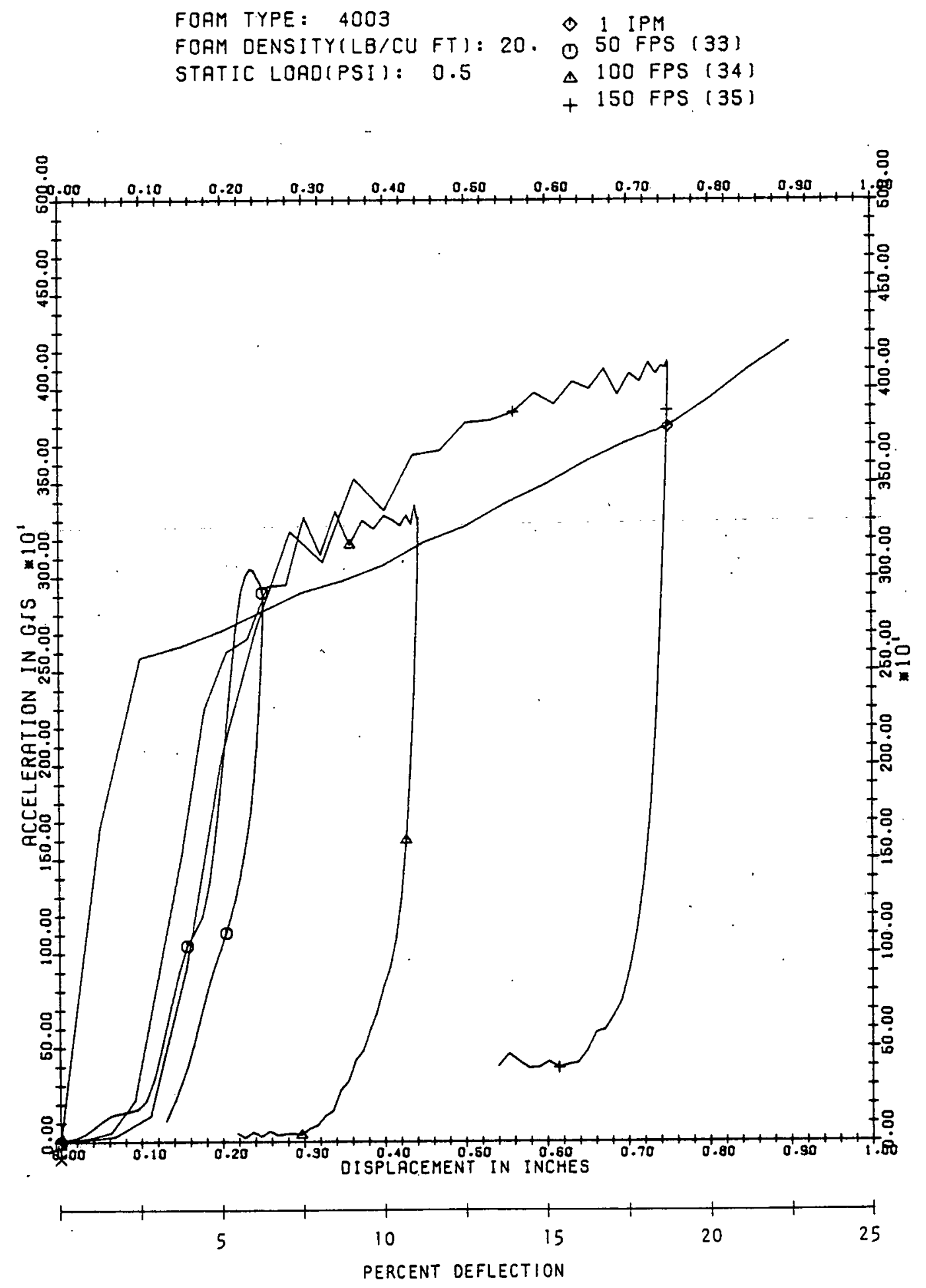

Figure B-25. BX 4003, $20 \mathrm{LB} / \mathrm{FT}^{3}, 0.5 \mathrm{PSI}$ 
ACCELERATION VS DISPLACEMENT

FOAM TYPE : 4003

FOAM DENSITYILB/CU FTI: 20. O 50 FPS (1921

STATIC LOAO(PSI): $1.0 \quad \triangle 100$ FPS (49)

+150 FPS (43)

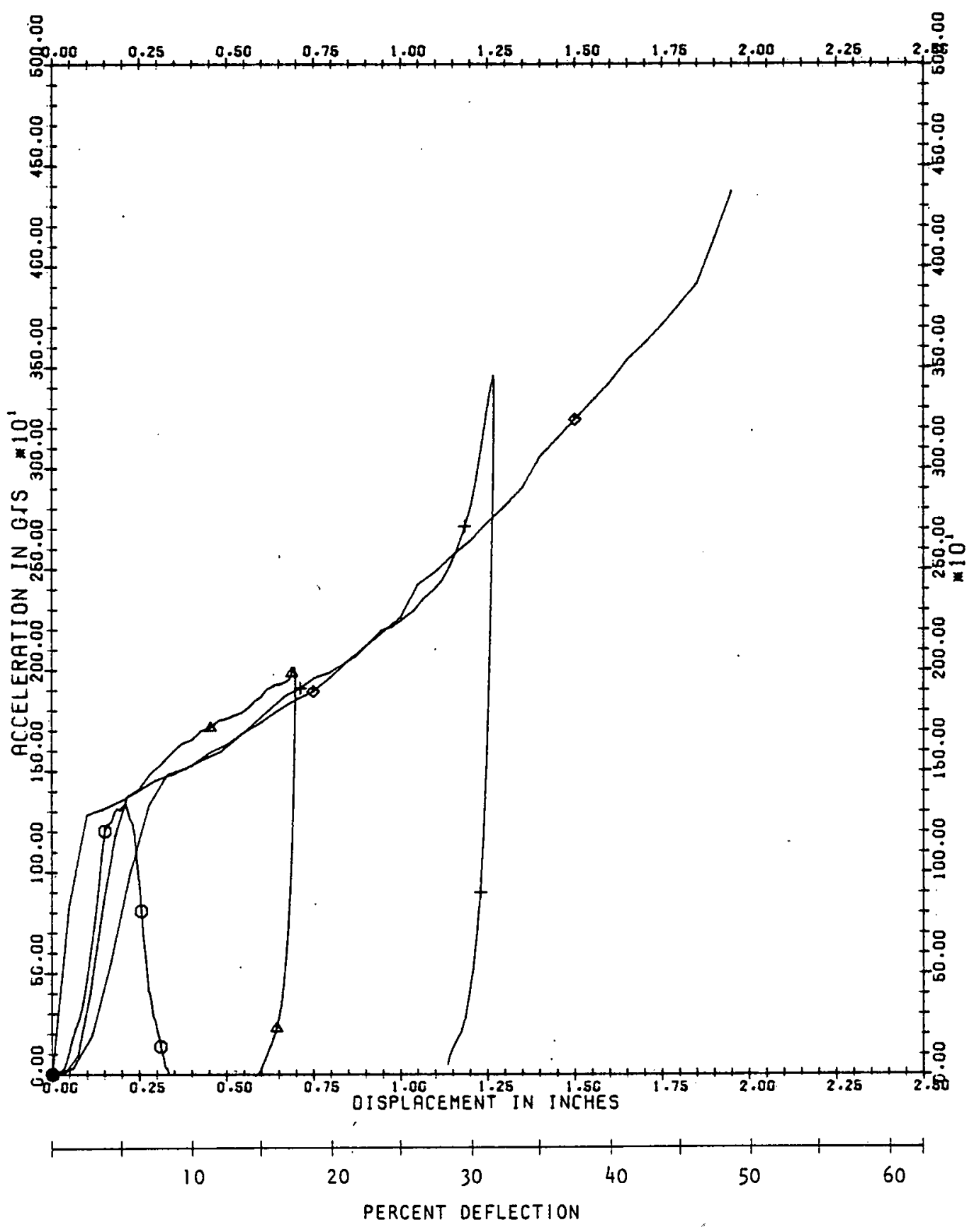

Figure B-26. BX 4003, $20 \mathrm{LB} / \mathrm{FT}^{3}, 1.0 \mathrm{PSI}$ 

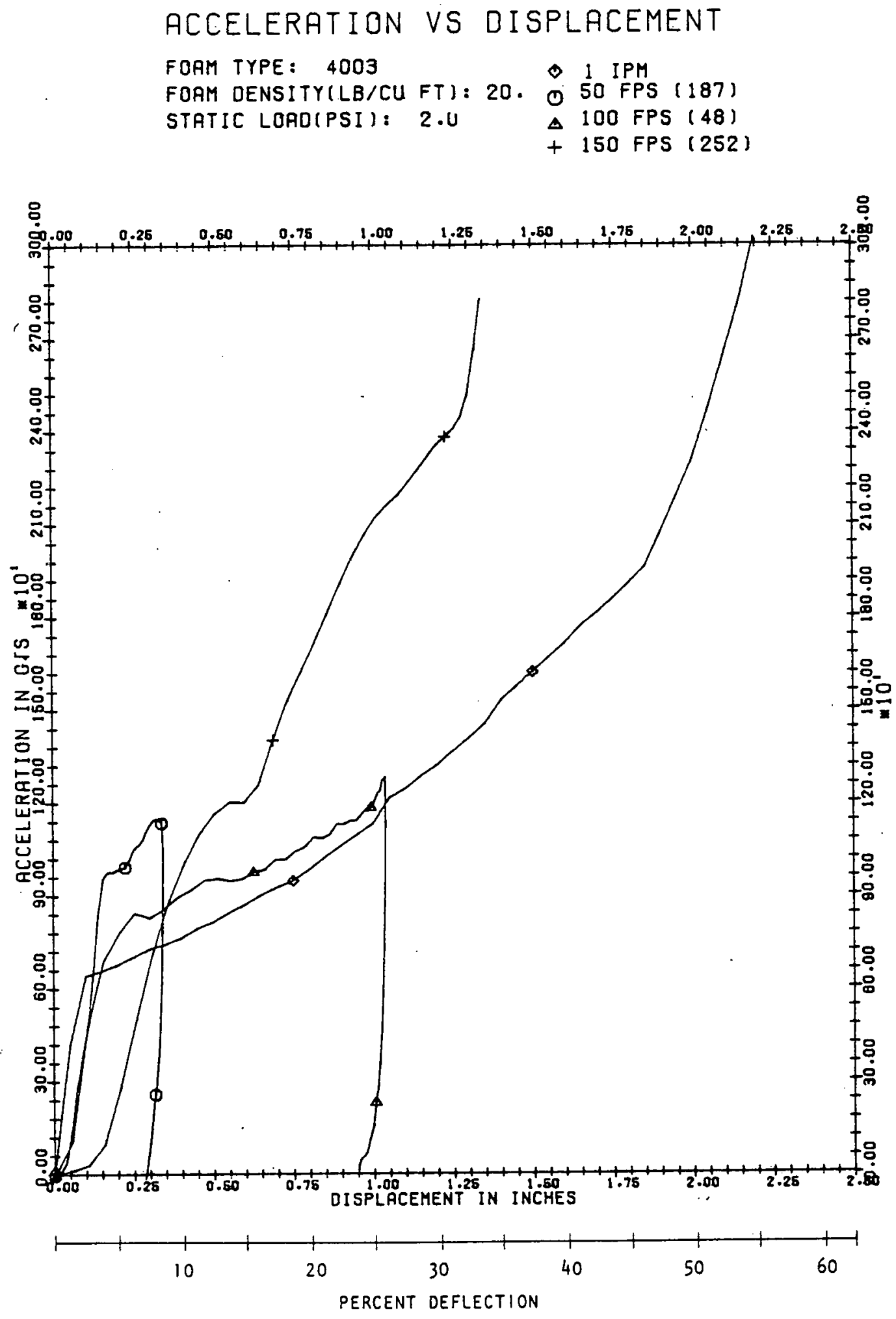

Figure B-27. BX 4003, $20 \mathrm{LB} / \mathrm{FT}^{3}, 2.0 \mathrm{PSI}$ 

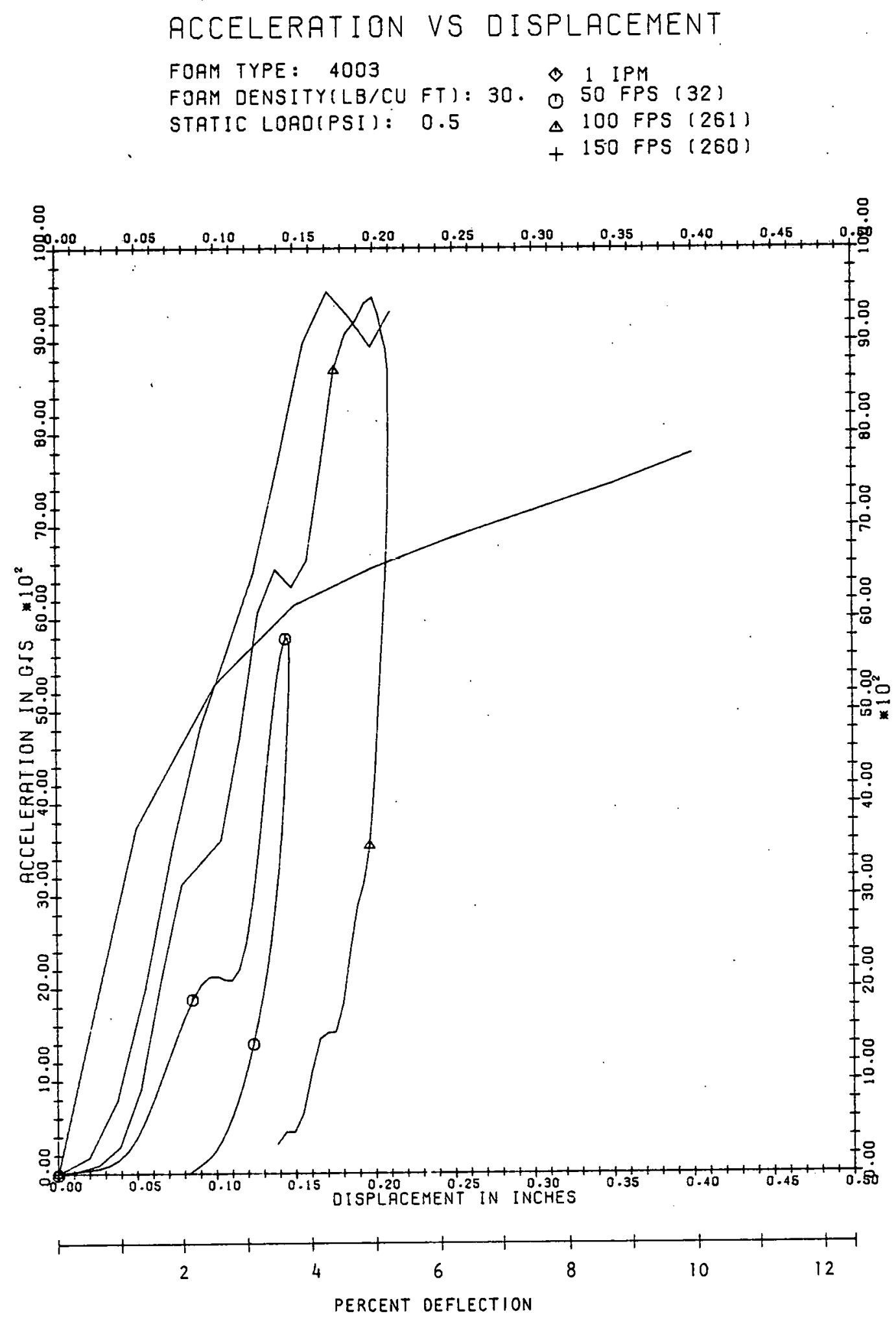

Figure B-28. BX 4003, $30 \mathrm{LB} / \mathrm{FT}^{3}, 0.5 \mathrm{pSI}$ 
ACCELERATION VS DISPLACEMENT
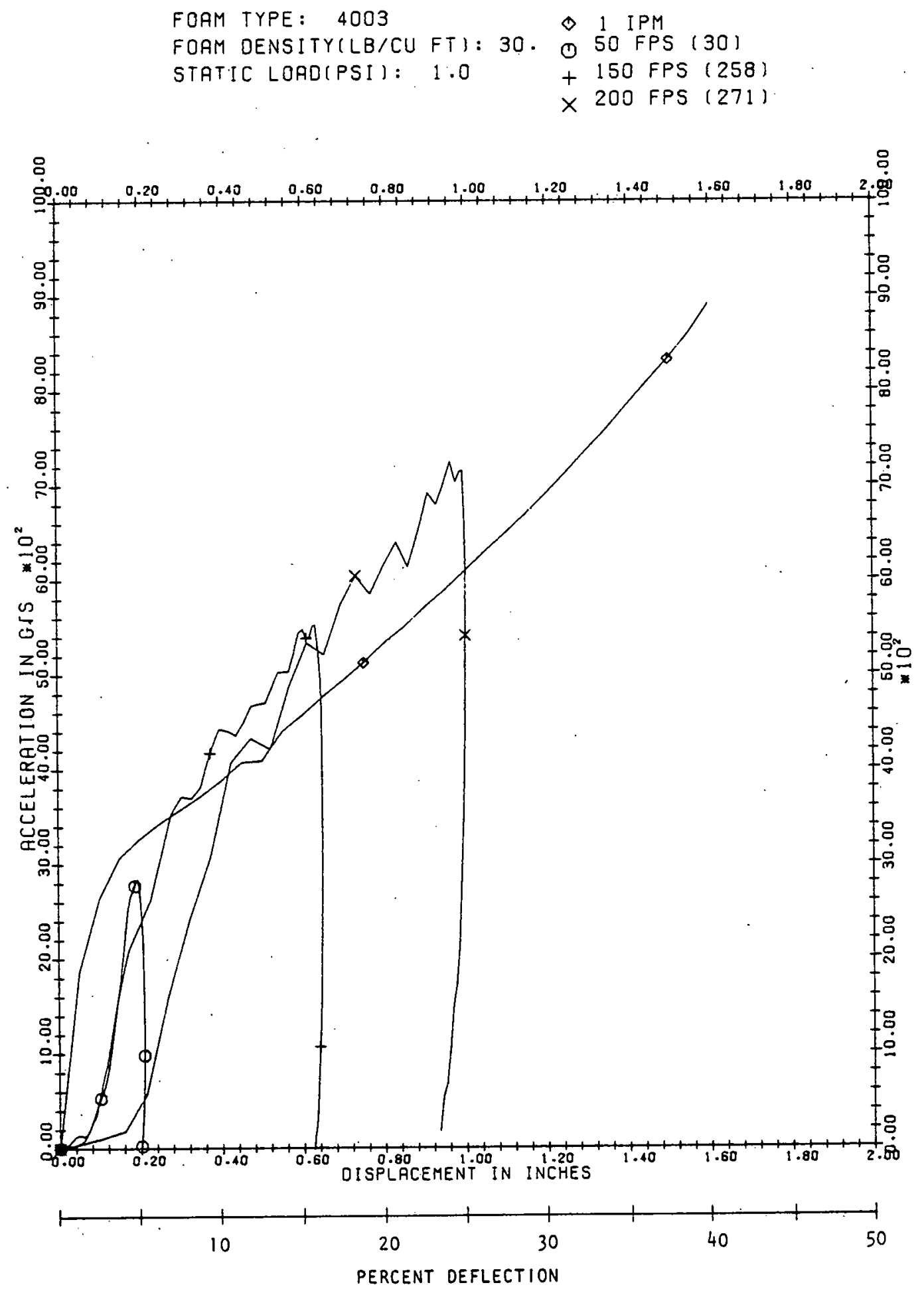

Figure B-29. BX $4003,30 \mathrm{LB} / \mathrm{FT}^{3}, 1.0 \mathrm{PSI}$ 
ACCELERATION VS DISPLACEMENT
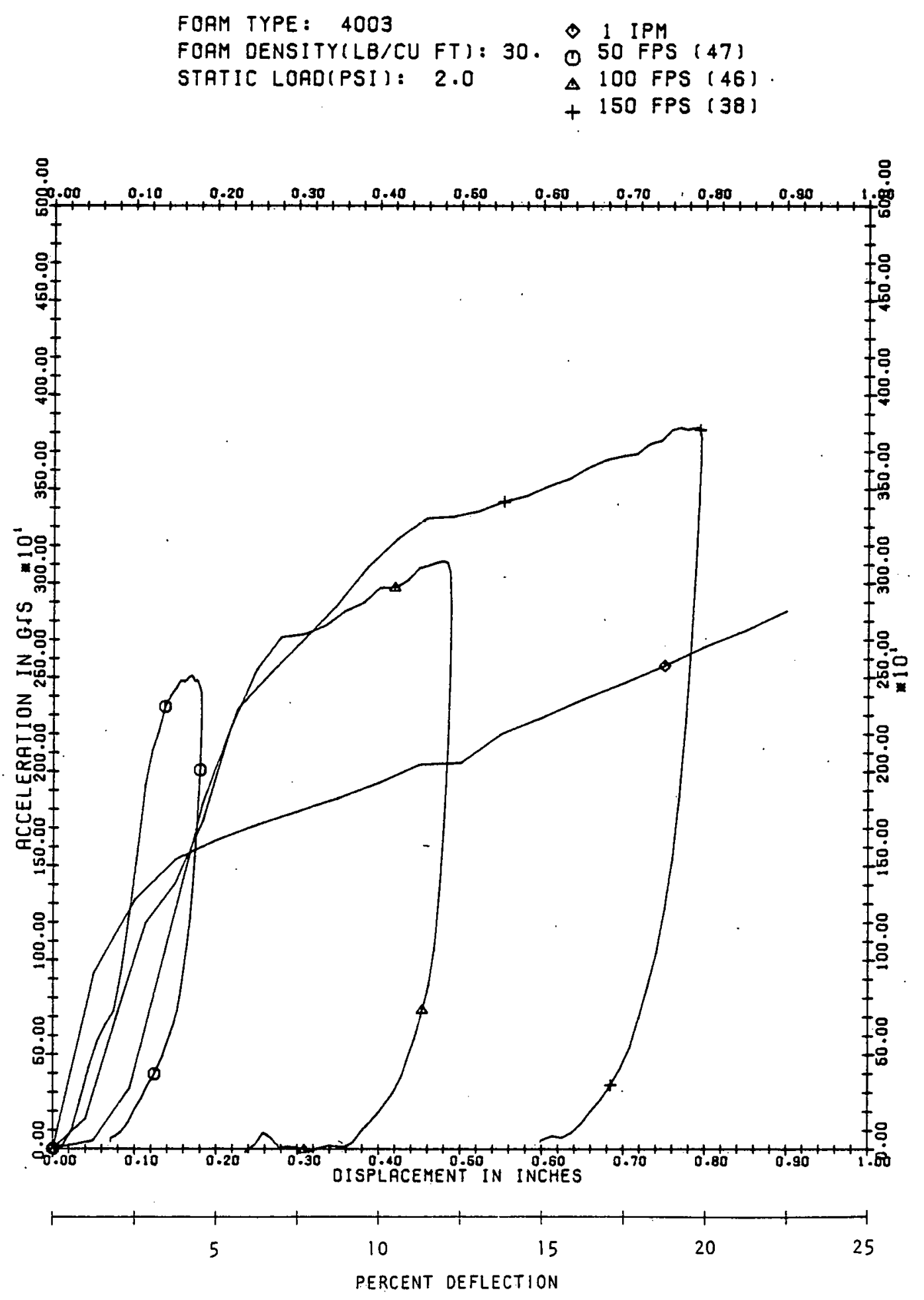

Figure B-30. BX 4003, $30 \mathrm{LB} / \mathrm{FT}^{3}, 2.0 \mathrm{PSI}$ 
ACCELERATION VS DISPLACEMENT
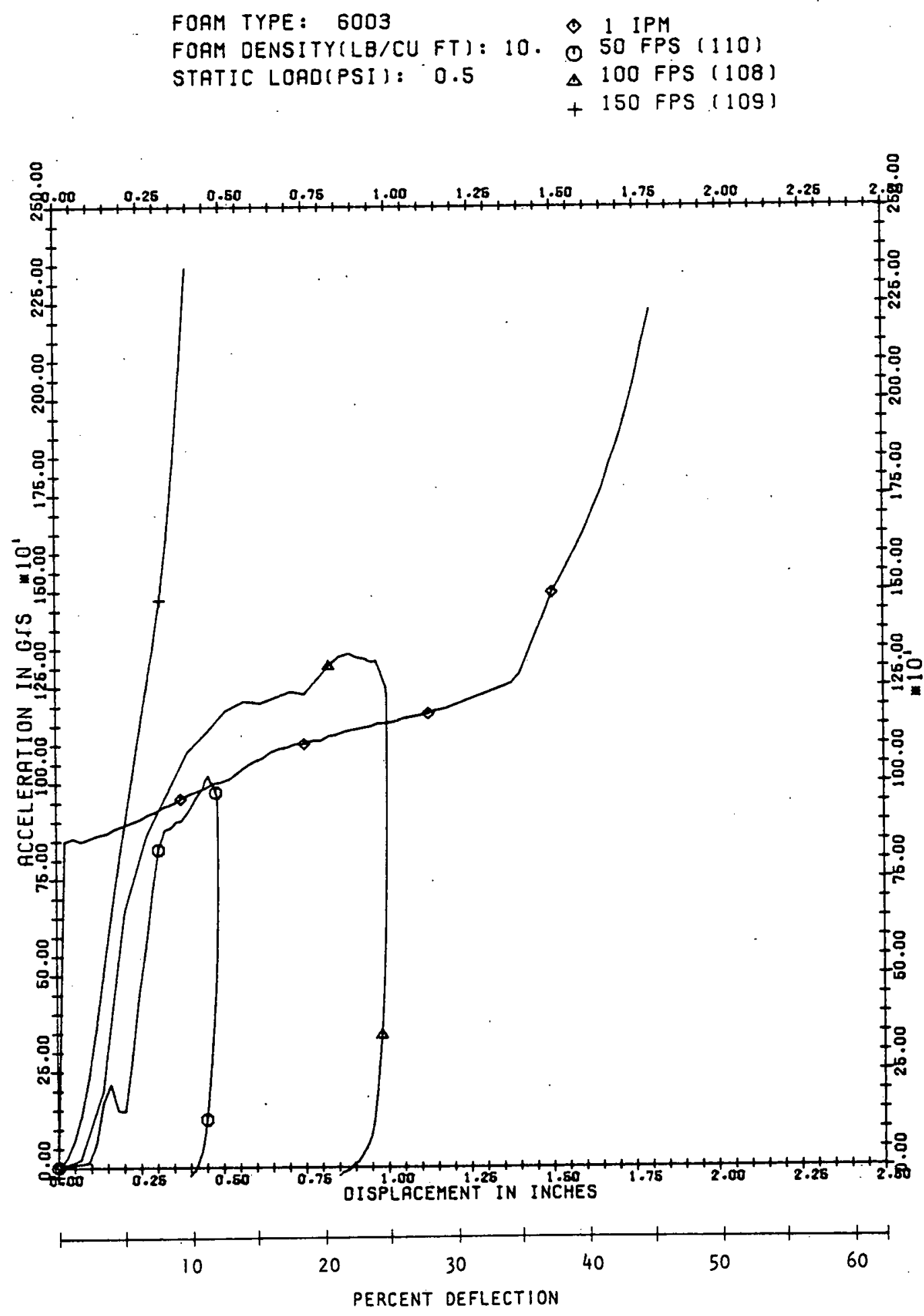

Figure B-31. BX 6003, $10 \mathrm{LB} / \mathrm{FT}^{3}, 0.5 \mathrm{PSI}$ 

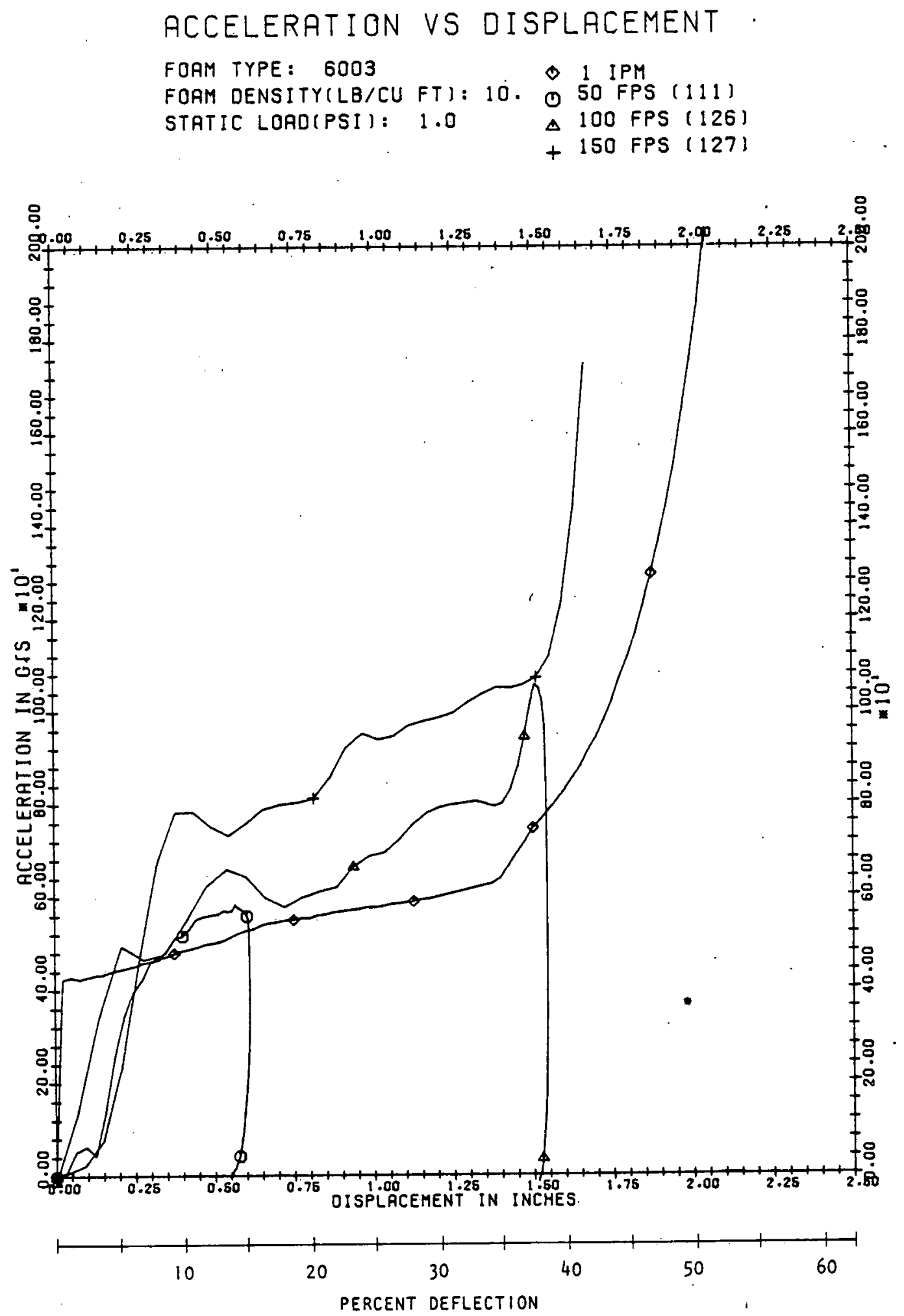

Figure B-32. BX 6003, $10 \mathrm{LB} / \mathrm{FT}^{3}, 1.0 \mathrm{PSI}$ 
ACCELERATION VS DISPLACEMENT
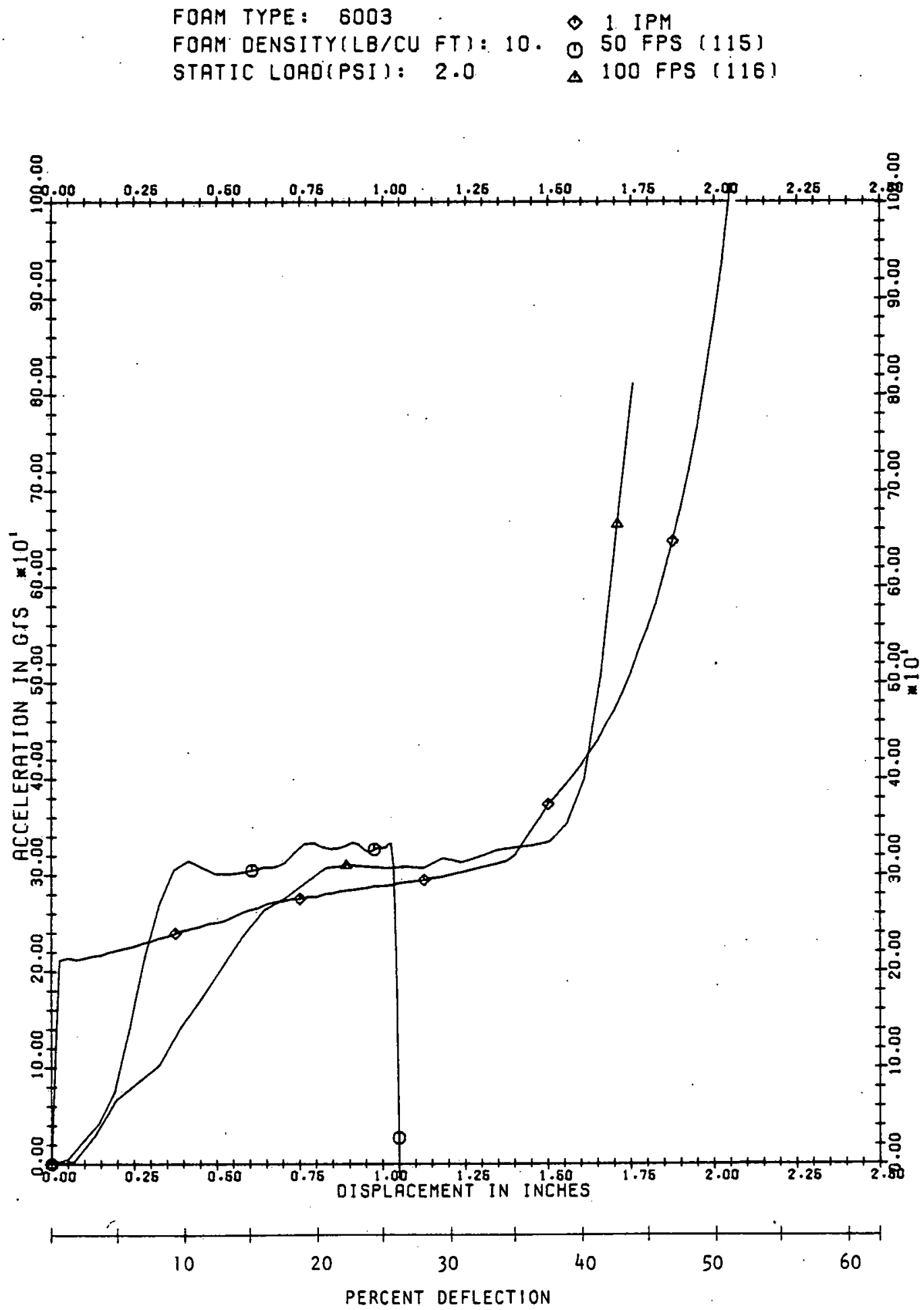

Figure B-33. BX 6003, $10 \mathrm{LB} / \mathrm{FT}^{3}, 2.0 \mathrm{PSI}$ 
ACCELERATION VS DISPLACEMENT

FORM TYPE: 6003

FOAM DENSITY(LB/CU FT): 20. 0.50 FPS (206)

STATIC LORO(PSI): $0.5 \quad \triangle 100$ FPS (219)

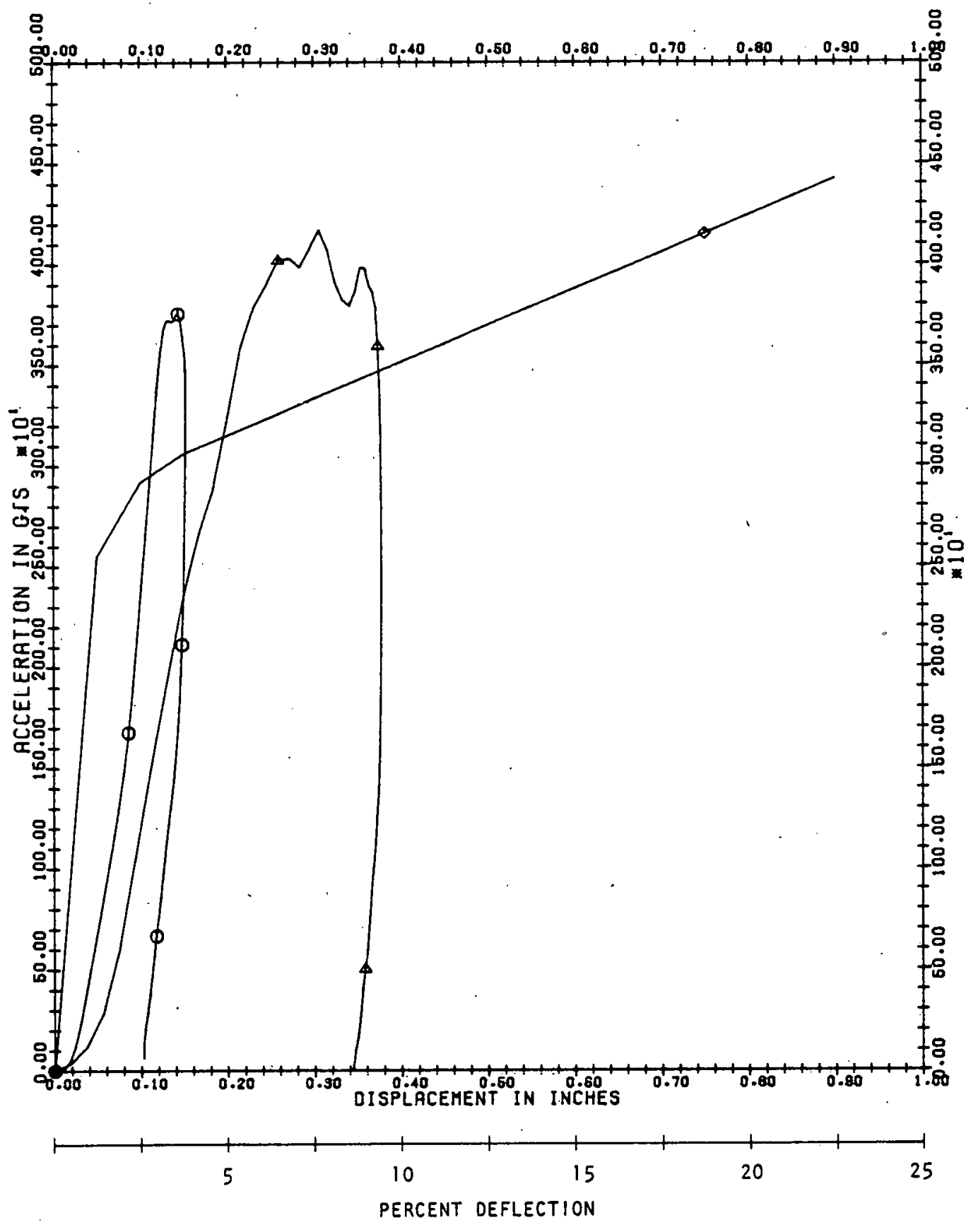

Figure B-34. BX 6003, $20 \mathrm{LB} / \mathrm{FT}^{3}, 0.5 \mathrm{PSI}$ 

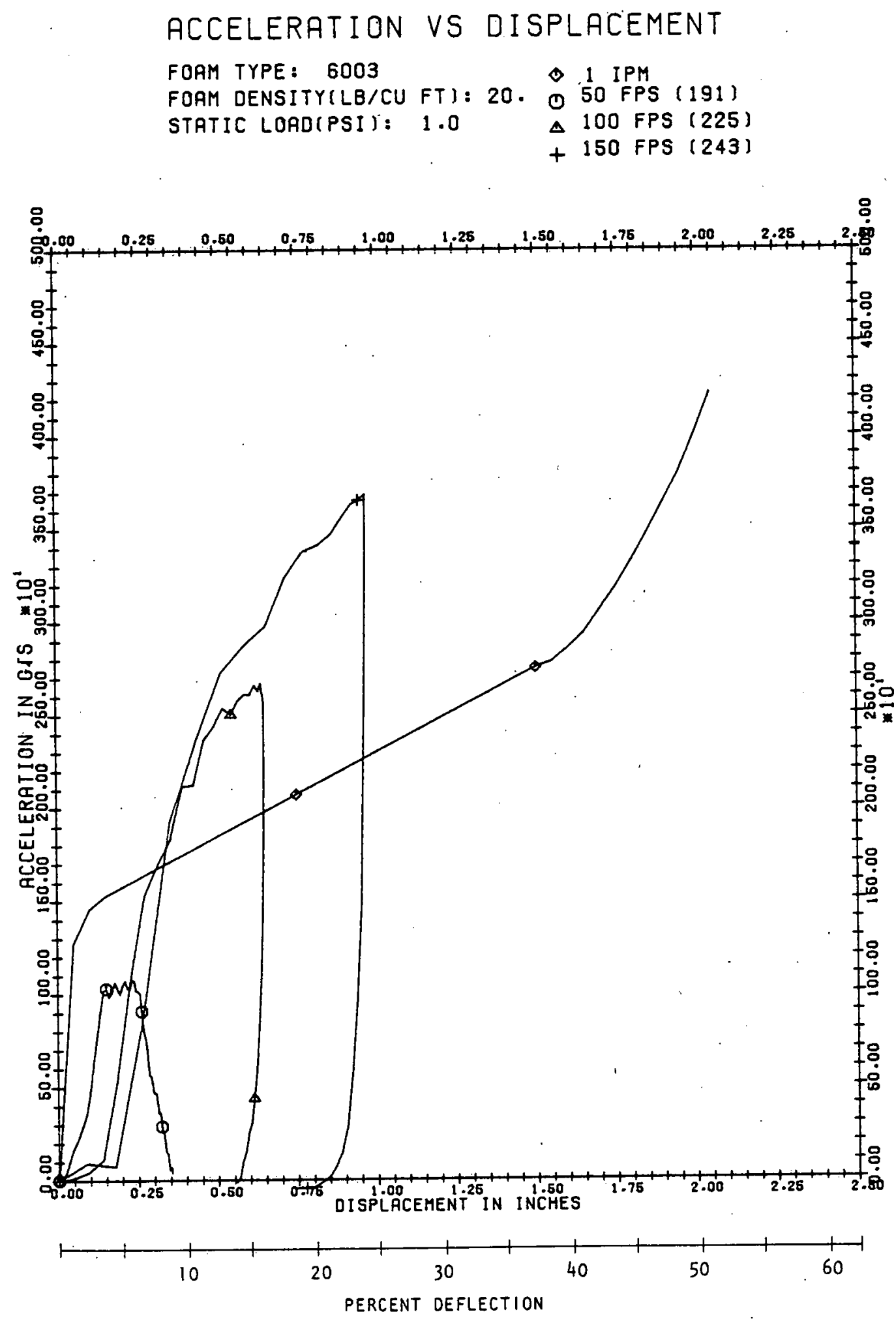

Figure B-35. BX 6003, $20 \mathrm{LB} / \mathrm{FT}^{3}, 1.0 \mathrm{PSI}$ 
ACCELERATION VS DISPLACEMENT

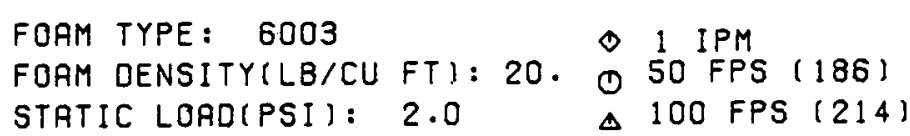

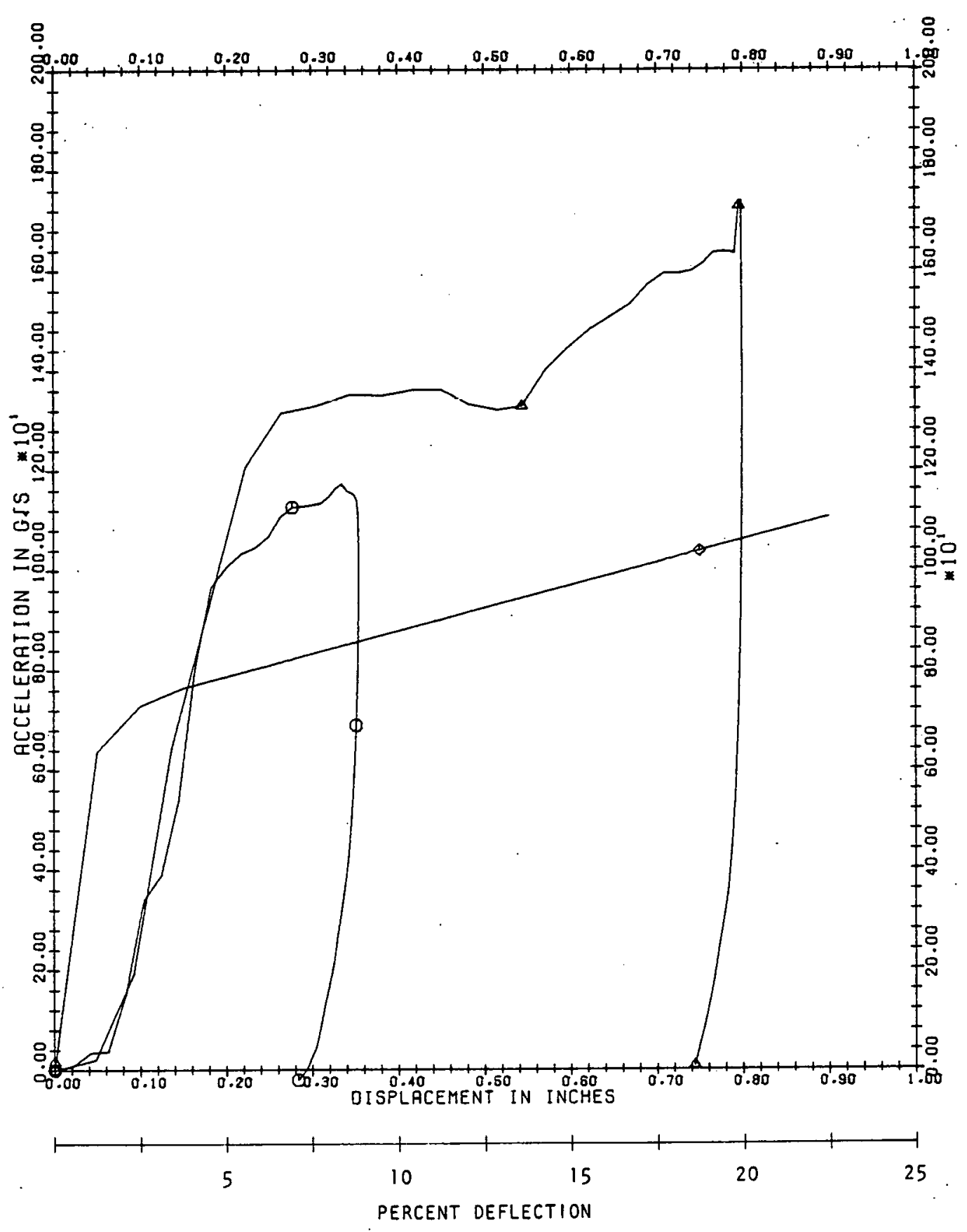

Figure B-36. BX $6003,20 \mathrm{LB} / \mathrm{FT}^{3}, 2.0 \mathrm{PSI}$ 
ACCELERATION VS OISPLACEMENT
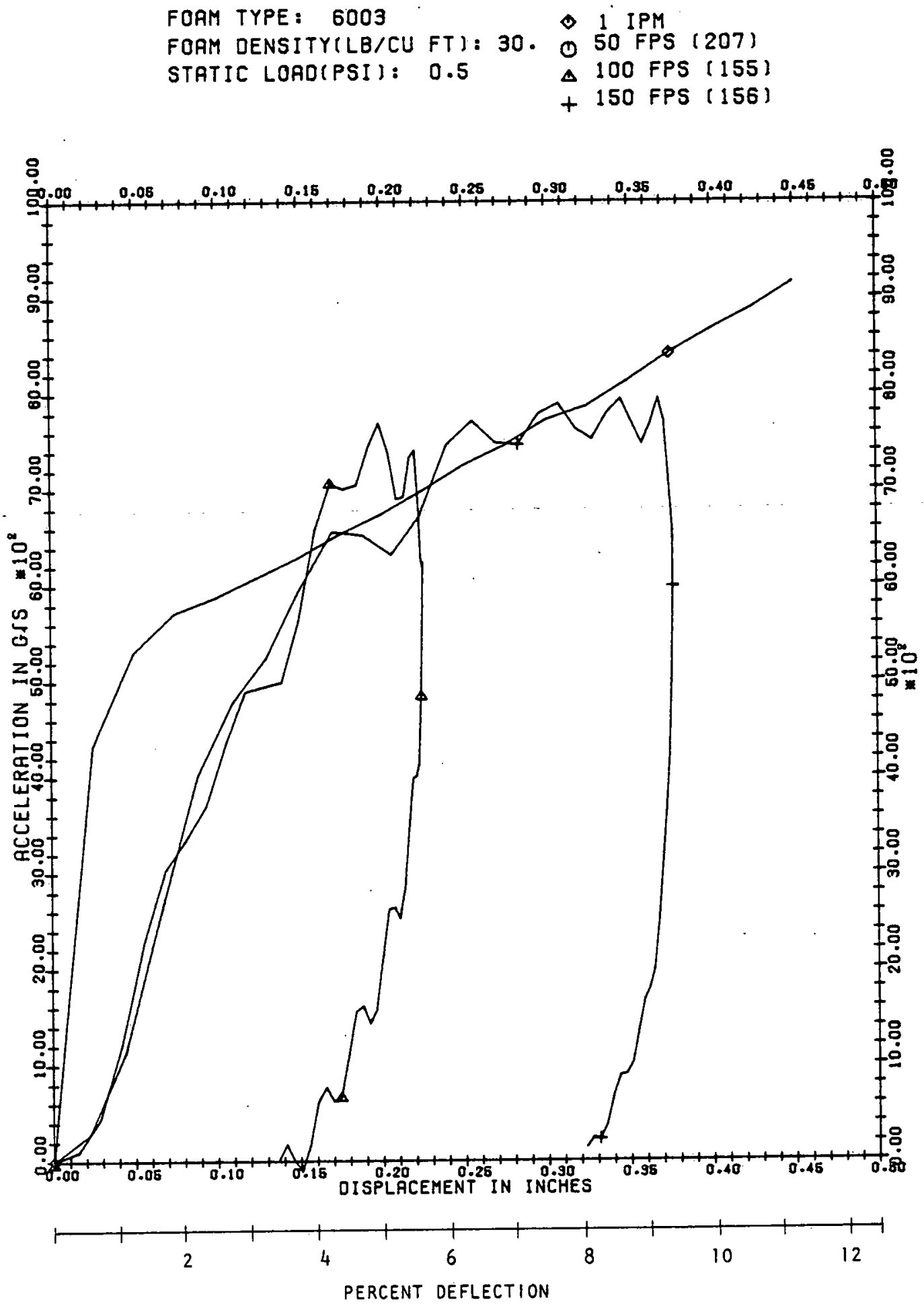

Figure B-37. BX 6003, $30 \mathrm{LB} / \mathrm{FT}^{3}, 0.5 \mathrm{PSI}$ 

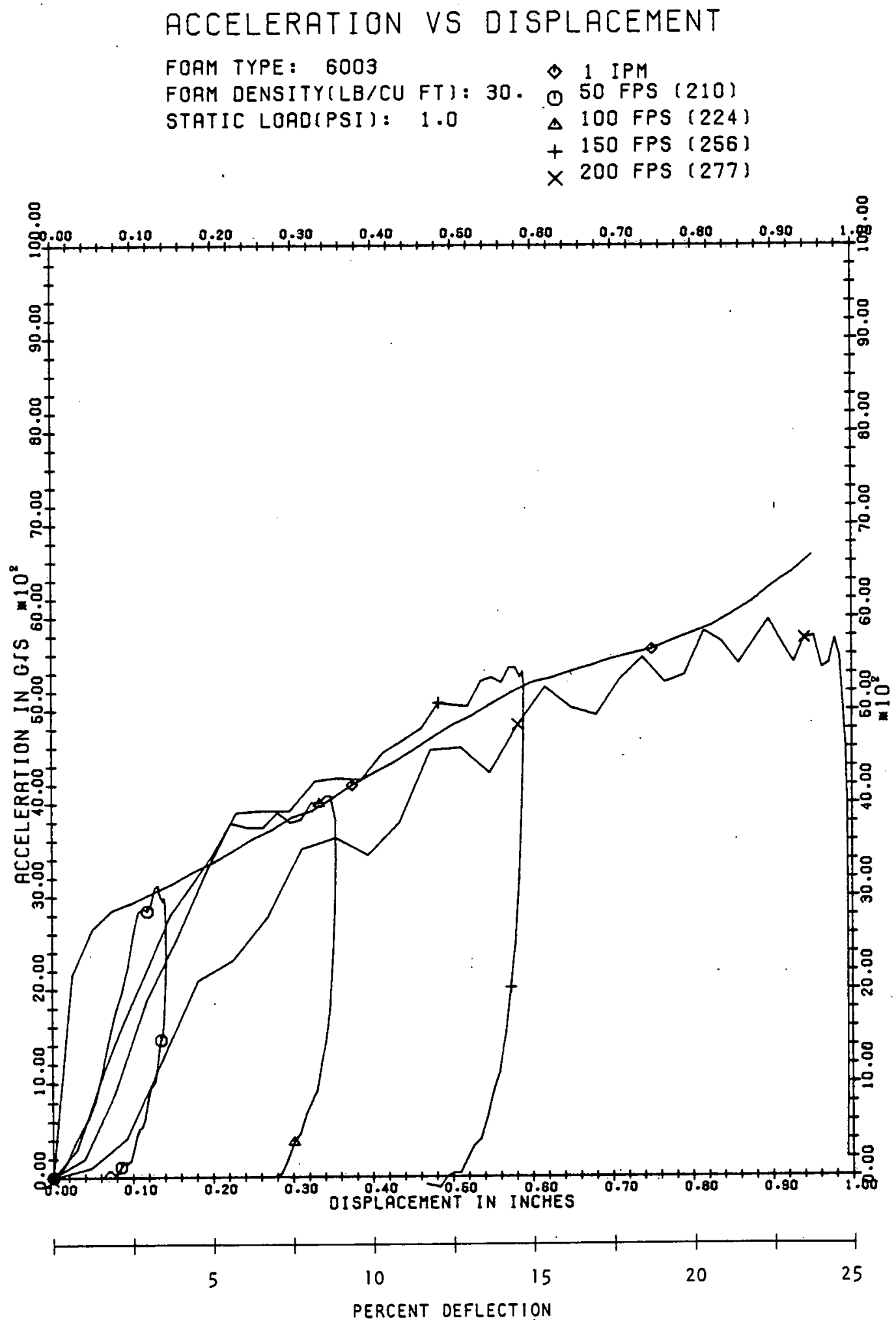

Figure B-38. BX 6003, $30 \mathrm{LB} / \mathrm{FT}^{3}, 1.0 \mathrm{PSI}$ 


\section{ACCELERATION VS DISPLACEMENT}
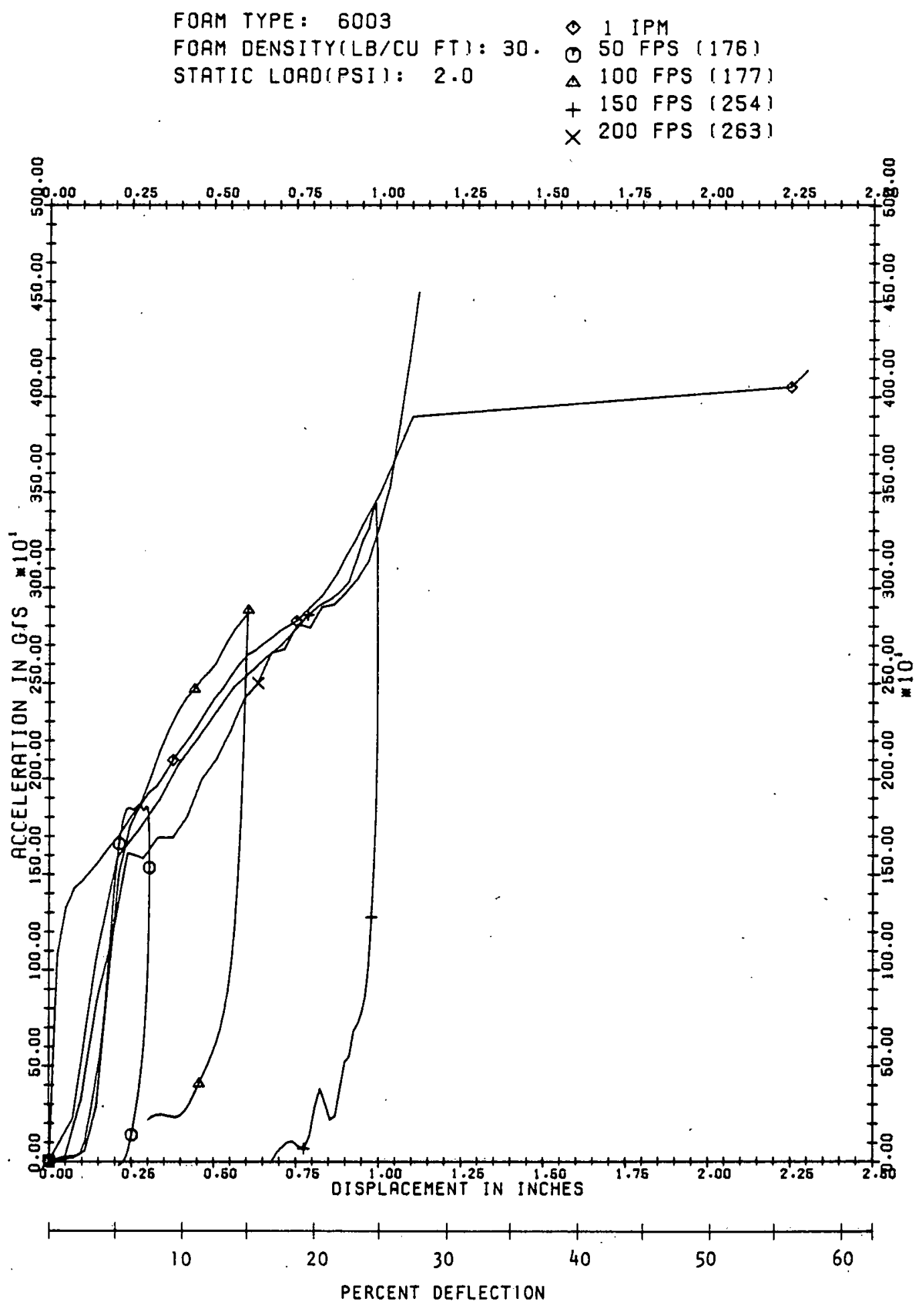

Figure B-39. BX 6003, $30 \mathrm{LB} / \mathrm{FT}^{3}, 2.0 \mathrm{PSI}$ 
. ACCELERATION VS DISPLACEMENT
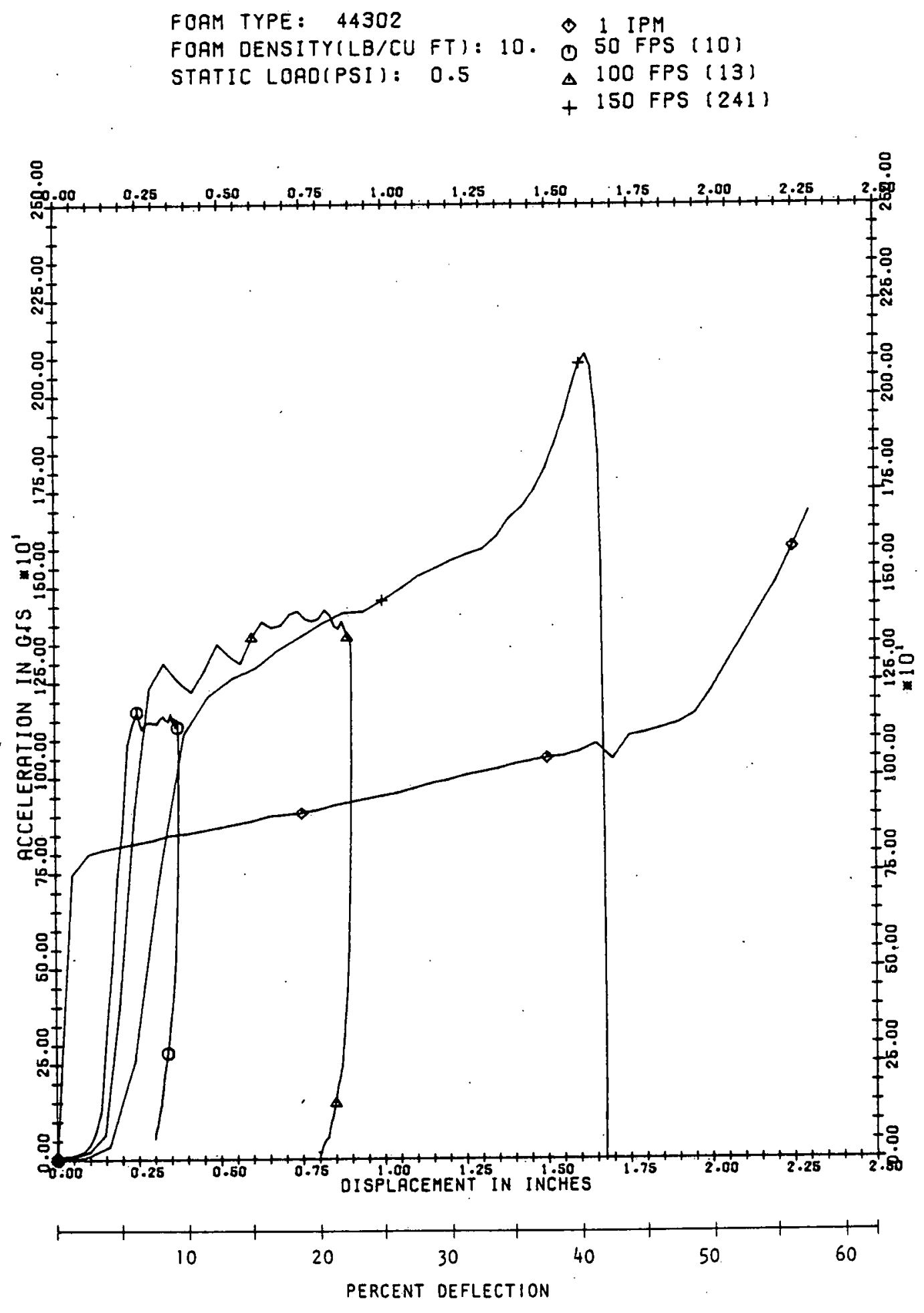

Figure B-40. BX 44302, $10 \mathrm{LB} / \mathrm{FT}^{3}, 0.5 \mathrm{PSI}$ 


\section{ACCELERATION VS DISPLACEMENT}

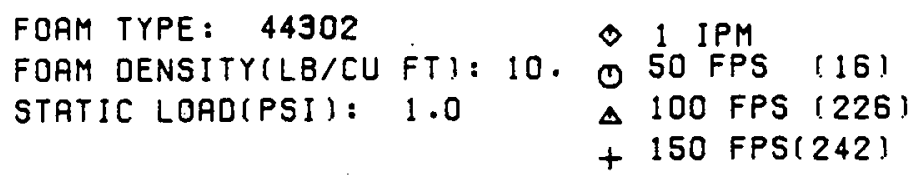

- 1 IPM

O 50 FPS $(16)$

$\triangle 100$ FPS (226)

+150 FPS (242)

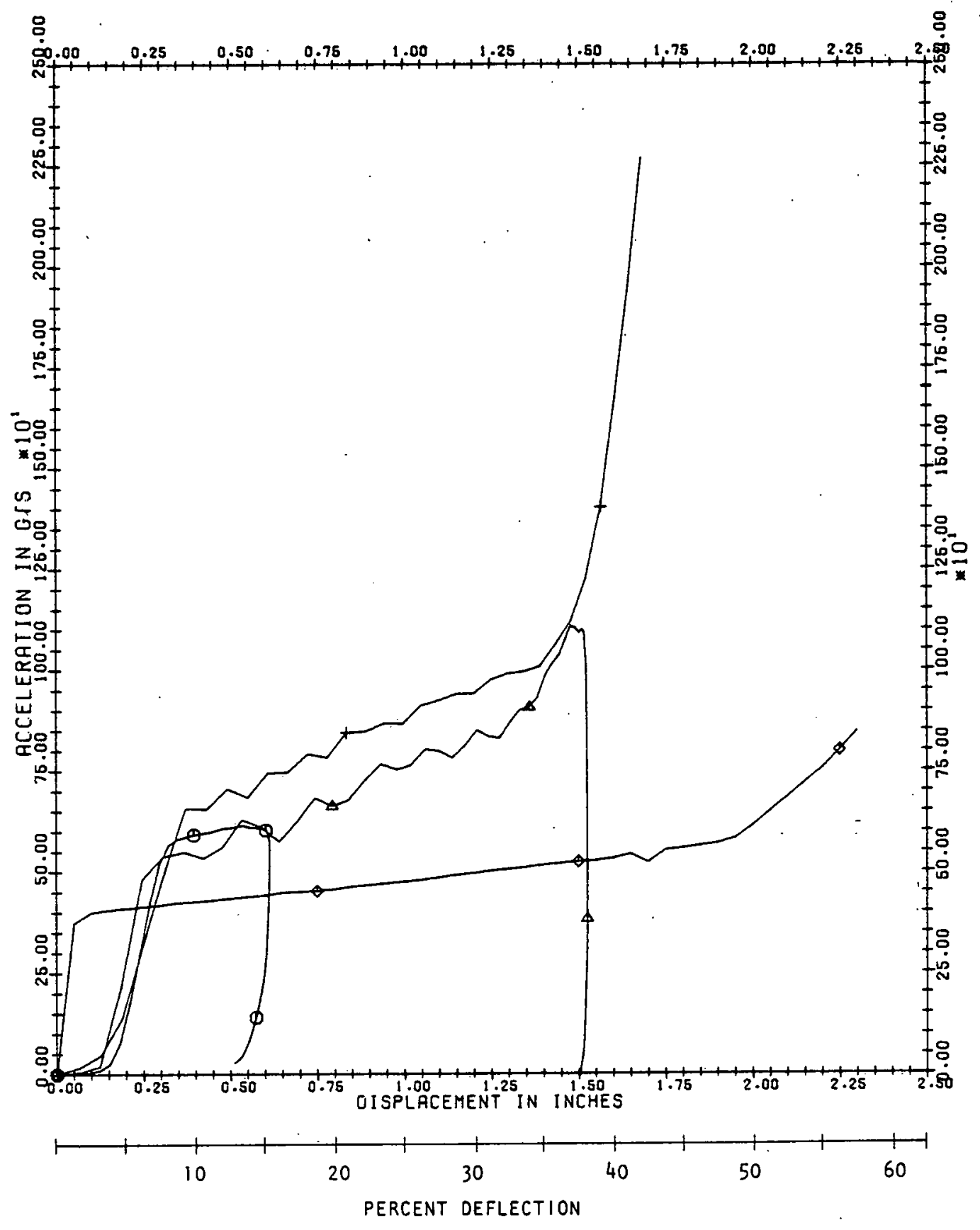

Figure B-41. BX $44302,10 \mathrm{LB} / \mathrm{FT}^{3}, 1.0 \mathrm{PSI}$ 


\section{ACCELERATION VS DISPLACEMENT}

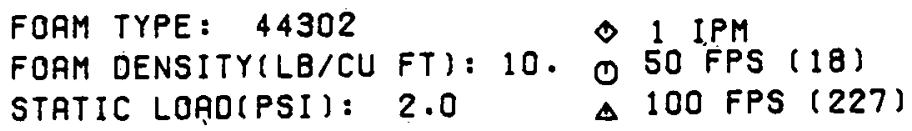

- 1 IPM

0 50 FPS (18)

$\triangle 100$ FPS (227)

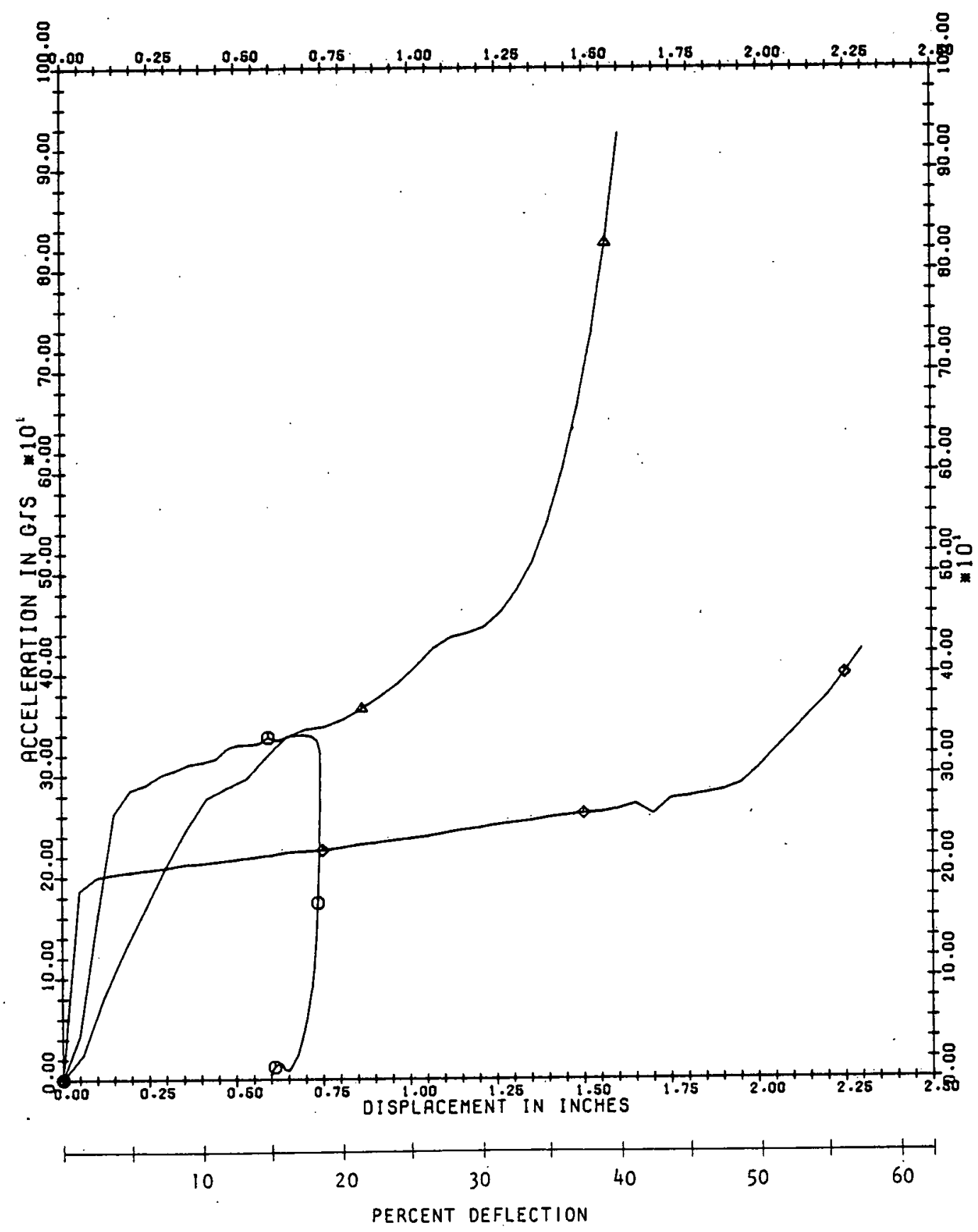

Figure B-42. BX $44302,10 \mathrm{LB} / \mathrm{FT}^{3}, 2.0 \mathrm{PSI}$ 
ACCELERATION VS DISPLACEMENT
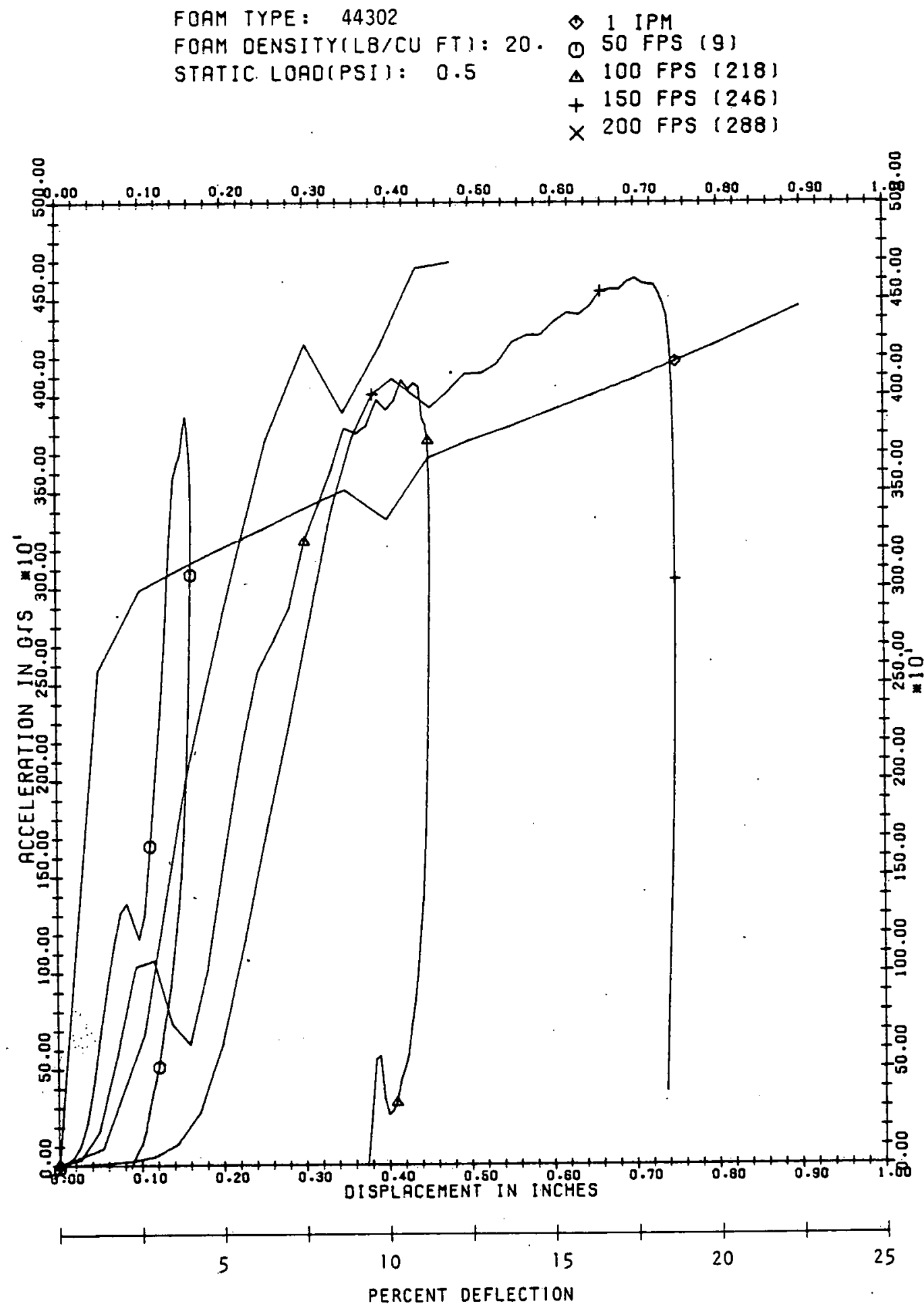

Figure B-43. BX 44302, $20 \mathrm{LB} / \mathrm{FT}^{3}, 0.5 \mathrm{PSI}$ 
ACCELERATION VS DISPLACEMENT
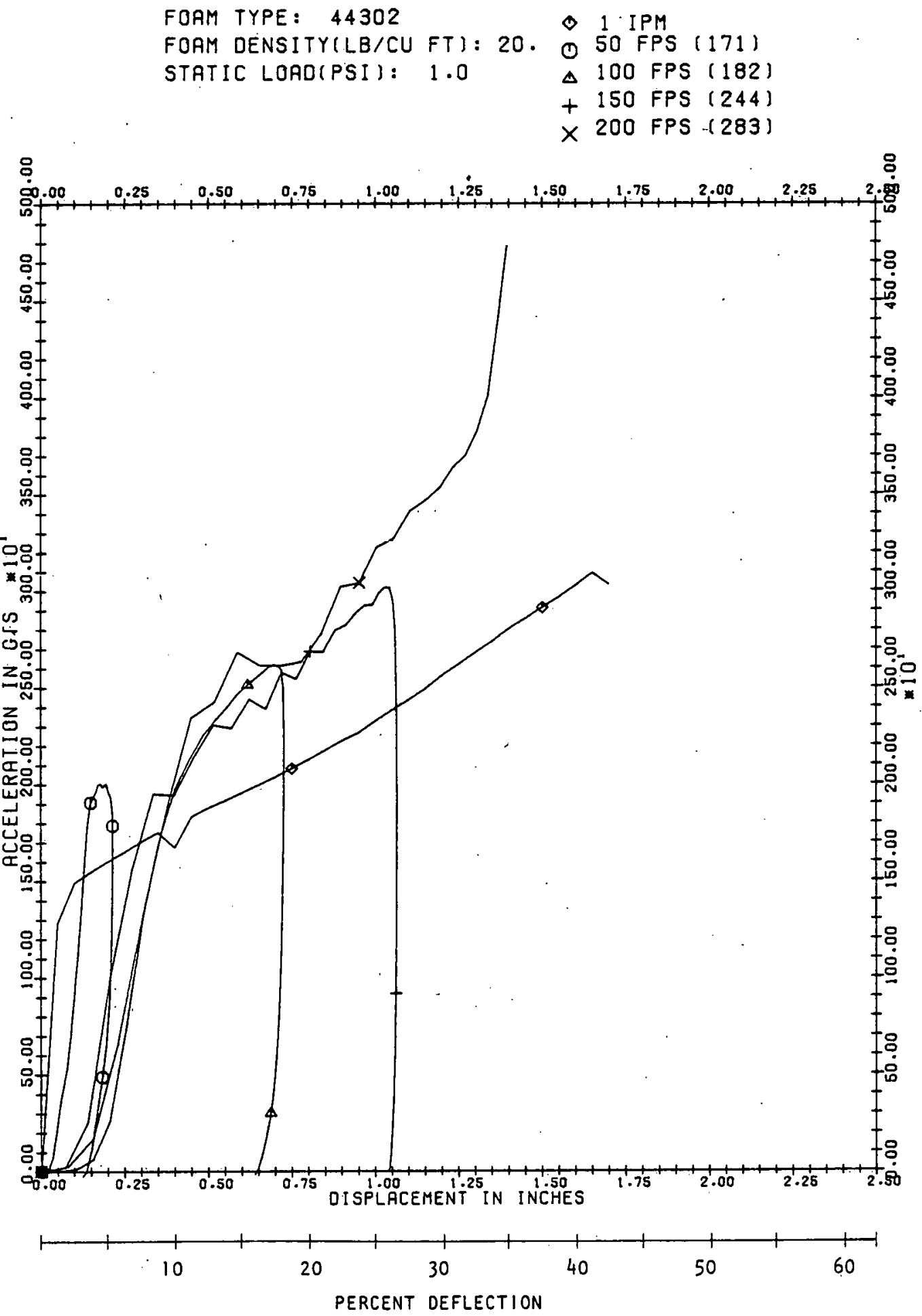

Figure B-44. BX 44302, $20 \mathrm{LB} / \mathrm{FT}^{3}, 1.0 \mathrm{PSI}$ 


\section{ACCELERATION VS DISPLACEMENT}
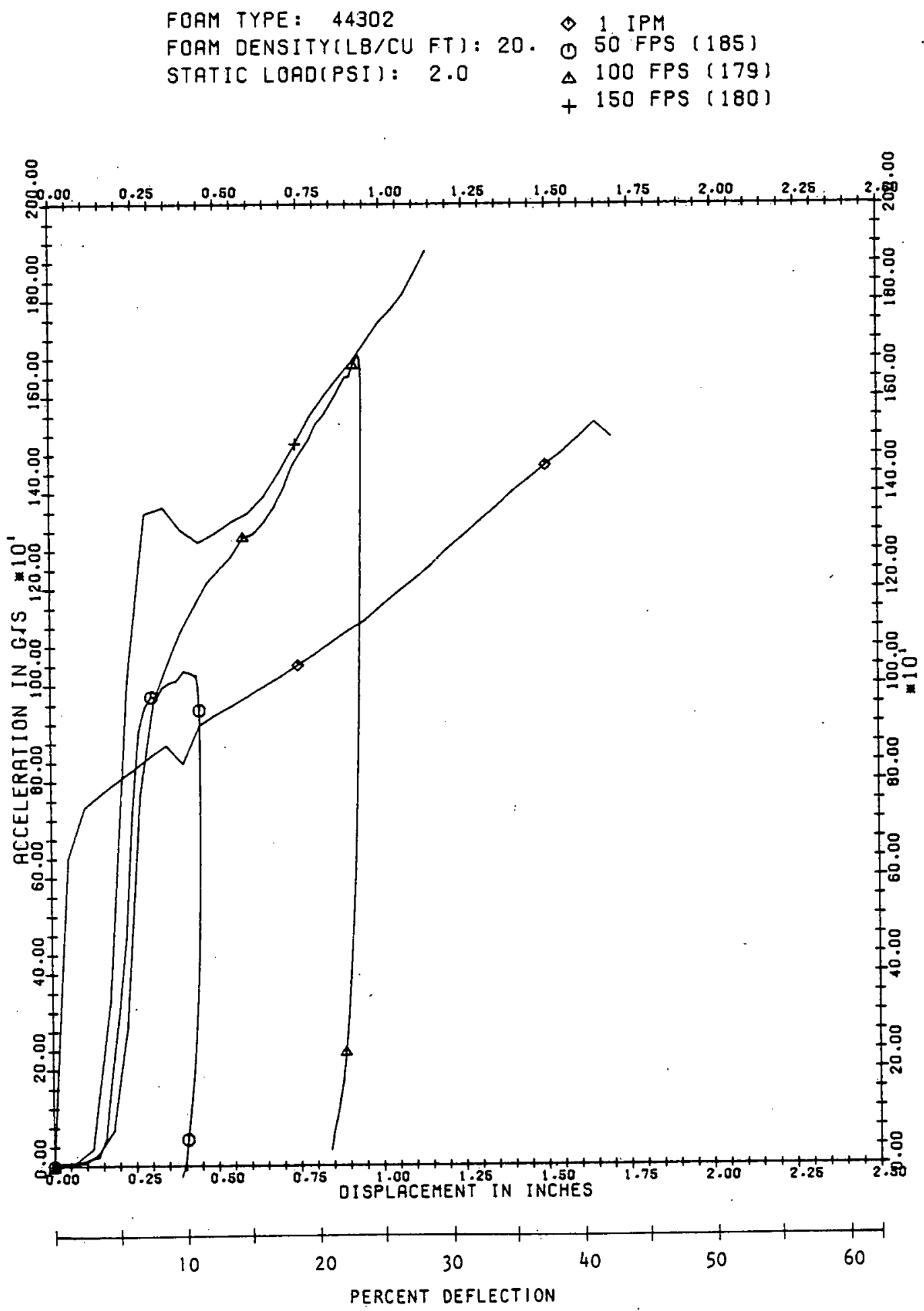

Figure B-45. BX 44302, $20 \mathrm{LB} / \mathrm{FT}^{3}, 2.0 \mathrm{PSI}$ 
ACCELERATTION VS DISPLACEMENT
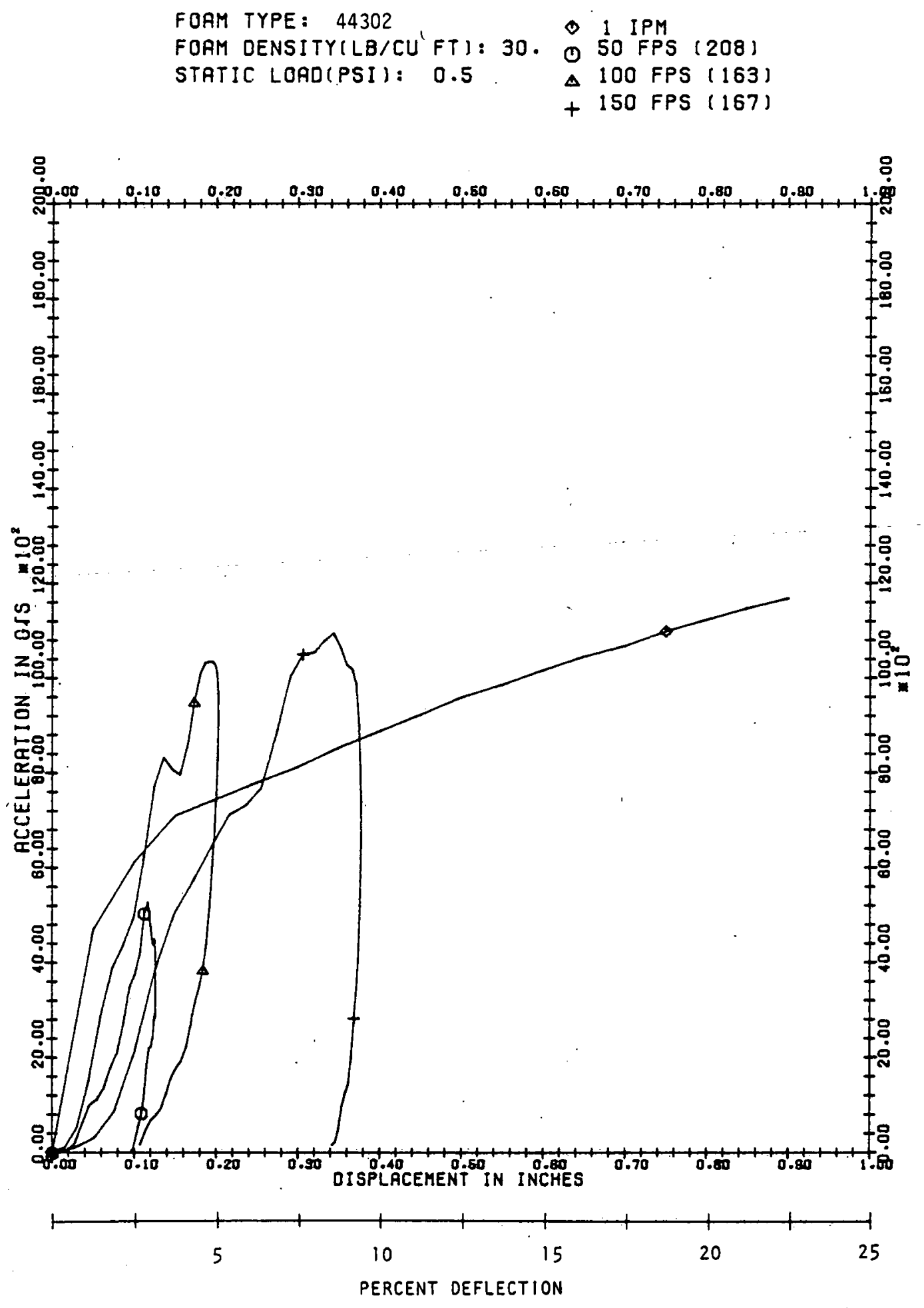

Figure B-46. BX 44302, $30 \mathrm{LB} / \mathrm{FT}^{3}, 0.5 \mathrm{PSI}$ 


\section{ACCELERATION VS DISPLACEMENT}
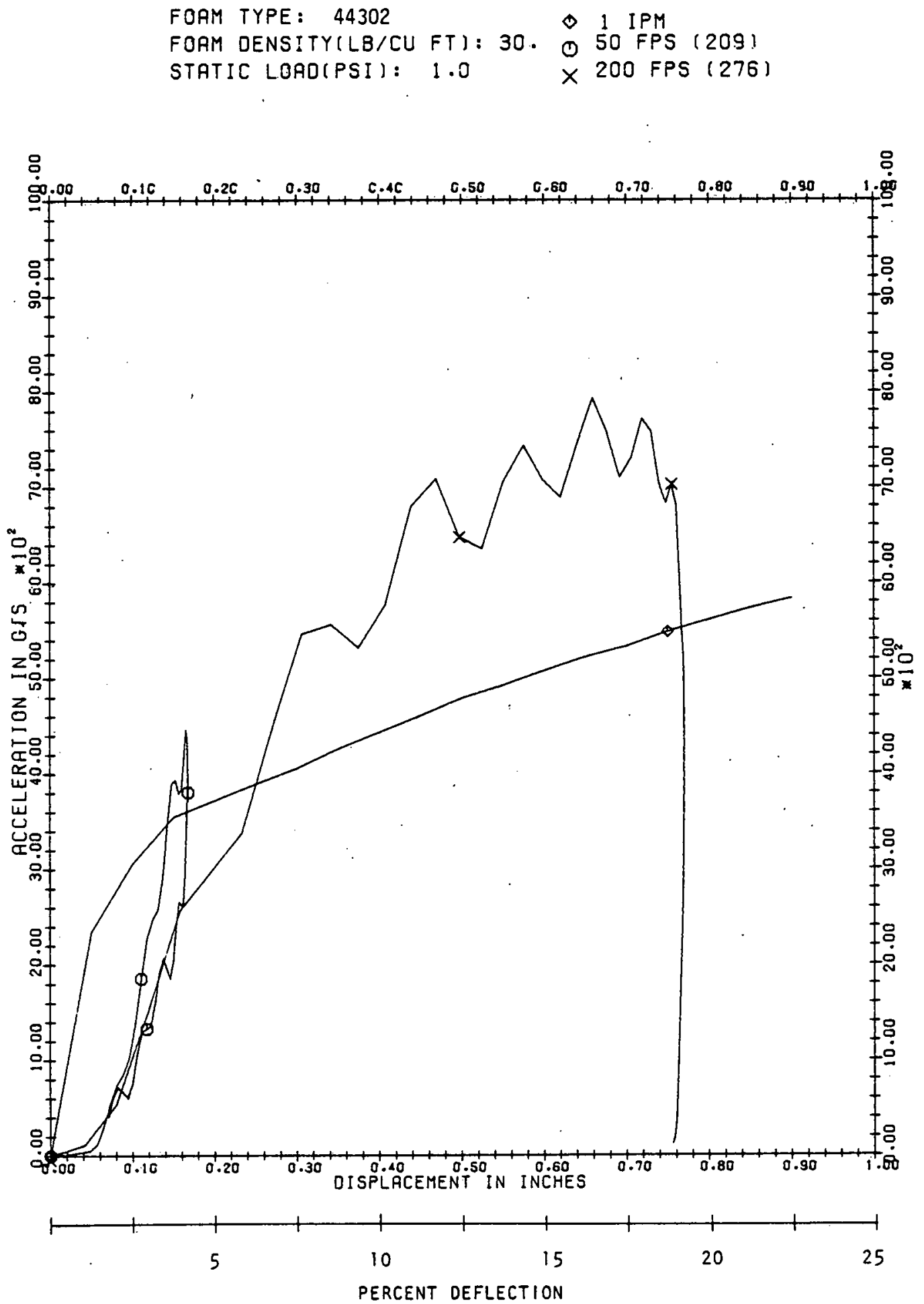

Figure B-47. BX $44302,30 \mathrm{LB} / \mathrm{FT}^{3}, 1.0 \mathrm{PSI}$ 
ACCELERATION VS DISPLACEMENT
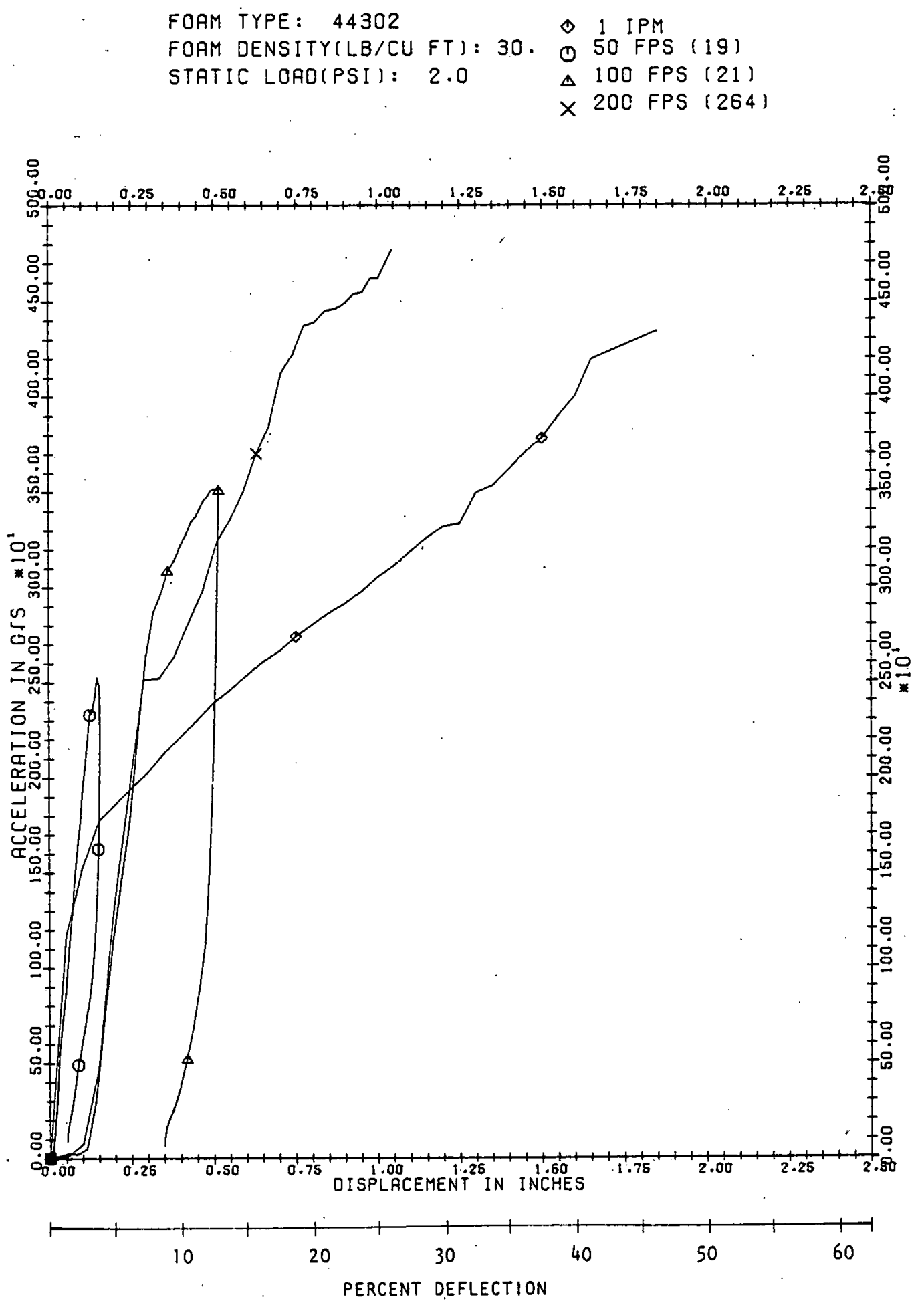

Figure $\mathrm{B}-48$. BX $44302,30 \mathrm{LB} / \mathrm{FT}^{3}, 2.0 \mathrm{PSI}$ 


\section{DISTRIBUTION}

Copy

R. Bulcock, ERDA-KCAO

1

V. C. Vespe, ERDA-ALO

F. W. DuBois, LASL

J. A. Freed, LASL

S. J. Buginas, LLL

A. B. Copeland, LLL

H. M. Brinkmeier, Monsanto

D. R. Anderson, SLA

T. K. Hill/W. W. Joseph, SLA

H. M. Jones/R. Grover, SLA

P. B. Rand, SLA

C. B. Frost, SLL

L. F. Thorne/R. D. Jump, D/144, SA8

J. D. Corey, D/554, BD50

L. Stratton, D/554, 2C44

R. F. Pippert, D/700, 1A42

R. P. Frohmberg, D/800, 2A39

D. H. $\mathrm{Hax}, \mathrm{D} / 800,2 \mathrm{~A} 41$

C. H. Smith/S. L. DeGisi/T. E. Neet, D/814, 2C43

V. E. Alley/G. E. Martinette/J. R. Fender/

R. C. Swoboda, D/861, 2A31

R. A. Daniel, D/861, 2A31

R. H. Graham, D/861, 2A31

R. E. Kessler, D/865, 2C40 